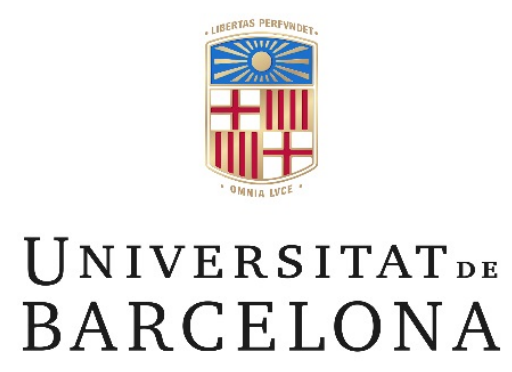

\title{
The Logic of Turing Progressions
}

Eduardo Hermo Reyes

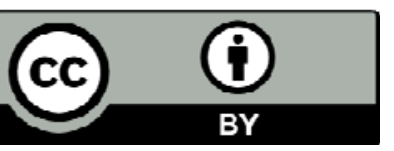

Aquesta tesi doctoral està subjecta a la llicència Reconeixement 4.0. Espanya de Creative Commons.

Esta tesis doctoral está sujeta a la licencia Reconocimiento 4.0. España de Creative Commons.

This doctoral thesis is licensed under the Creative Commons Attribution 4.0. Spain License. 


\title{
The Logic of Turing Progressions
}

\author{
Eduardo Hermo Reyes \\ University of Barcelona \\ Ph.D. Program in Mathematics and Computer Science \\ 2019
}

Supervisors:

Joost J. Joosten and David Fernández-Duque

Tutor:

Ramon Jansana 

Behold the perfect pantheon of absence. 



\section{Contents}

Contents

Acknowledgements

Abstract $\quad$ V

Resumen

Introduction $\quad$ IX

I Preliminaries 1

1.1. The Language of Arithmetic . . . . . . . . . . . . . . . . 1

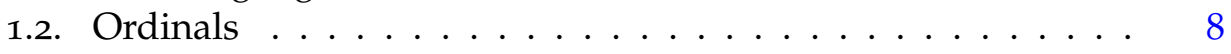

1.3. Turing progressions . . . . . . . . . . . . . 12

1.4. Provability $\operatorname{Logic} \ldots \ldots \ldots \ldots \ldots$

2 The Turing-Schmerl Calculus 21

2.1. Signature and Ordinal Modalities . . . . . . . . . . 21

2.2. The Turing-Schmerl Calculus . . . . . . . . . . . . 24

2.3. Normal Forms . . . . . . . . . . . . . . . . . 25

3 Arithmetical Semantics 33

3.1. Arithmetical interpretation of modal formulas . . . . . . . 33

3.2. Arithmetical Soundness of TSC . . . . . . . . . . . . 34

3.3. Completeness of TSC . . . . . . . . . . . . . . 44

4 Relational Semantics for TSC 51

4.1. A Variation on Ignatiev's Frame . . . . . . . . . . . . . 51

4.2. Modal Soundness and Completeness . . . . . . . . . . 66

5 More on TSC 73

5.1. A Finitely Supported Universal Frame . . . . . . . . . . . . 73

5.2. A calculus without normal forms . . . . . . . . . . . 79

5.3. Modal Schmerl principles . . . . . . . . . . . . . . 83 


\section{CONTENTS}

6 The Bracket Calculus $\quad 85$

6.1. Beklemishev's bracket notation system for $\Gamma_{0} \ldots \ldots \ldots$. . . 85

6.2. Signature . . . . . . . . . . . . . . . . 87

6.3. Translation and preservability . . . . . . . . . 89

$\begin{array}{ll}\text { Future Work } & 95\end{array}$

$\begin{array}{ll}\text { Bibliography } & 99\end{array}$ 


\section{Acknowledgements}

My first and deepest words of gratitude go to my supervisors Joost J. Joosten and David Fernández-Duque for their direction and patience along these years. With them I had my first contact in the study of proof theory, modal logic and ordinal analysis. They managed to awake my interest in these beautiful topics even when my personal lack of confidence in my understanding of these subjects blocked my progress. I learned from them that sometimes difficulties should be seen as opportunities.

I am very grateful to the logic group of Barcelona, in particular to all the professors that help me during my master studies and to all the participants of the proof theory and foundations of mathematics seminar and the $\mathrm{PhD}$ seminar in mathematical logic.

My gratitude goes to Lev Beklemishev. His comments and suggestions gave me a broader view of this work which turned out to be a substantial improvement. His deep view on these topics and the clarity of his expositions is something that I find inspiring. I wish to thank Fedor Pakhomov for his technical advice that refined my understanding of Schmerl's principles.

From a personal perspective, I would like to mention several people that made this work possible with their support and love. In Barcelona I came across colleagues who quickly became friends and family; many thanks to Pedro Andrés Esteban, Tommaso Moraschini and Amanda Vidal for their enlightenment and for every conversation and adventure we have gone through. Many thanks as well to my closest friends from Bilbao, Joseba, Gonzalo, Txipi, Endika and Sebas, for their constant support through all these years of changes, wise moves and stupid mistakes. A mis padres y, especialmente, a mi madre por todo su apoyo, escucha, comprensión y amor a lo largo de estos 32 años. Sin ella nada de esto hubiera sido posible. Moltes gràcies també a l'Alba per haver compartit tot des del moment en què vam coincidir i per tot el que encara ens queda per viure. T'estimo. 



\section{Abstract}

This dissertation is devoted to developing modal logical tools that can be used in the field of proof theory and ordinal analysis. More precisely, we focus on the relation between strictly positive modal logics and both Turing progressions and ordinal notation systems.

With respect to the former one, we introduce the system TSC that is tailored to generate exactly all relations that hold between different Turing progressions given a particular set of natural consistency notions. We also present an arithmetical interpretation for this modal system, named the Formalized Turing progressions interpretation. The logic is proven to be arithmetically sound and complete with respect to this interpretation.

After exploring the arithmetical semantics of TSC, we investigate the relational semantics of this system. For this purpose, we make use of the universal model of the closed fragment of Gödel-Löb's Polymodal Logic (GLP), namely Ignatiev's universal frame. By slightly modifying the relations defined in this model, we obtain a new frame which is proven to be a universal model for TSC. Moreover, we show how the domain of this frame can be reduced to sequences with finite support while keeping the completeness of the system.

As for ordinal notations systems, we present the logic BC (for Bracket Calculus). Unlike other provability logics, BC is based on a purely modal signature that gives rise to an ordinal notation system instead of modalities indexed by some ordinal given a priori. Moreover, since the order between these notations can be established in terms of derivability within the calculus, the inferences in this system can be carried out without using any external property of ordinals. The presented logic is proven to be equivalent to Reflection Calculus $\left(\mathbf{R C}_{\boldsymbol{\Gamma}_{0}}\right)$, that is, to the strictly positive fragment of $\mathbf{G L P}_{\Gamma_{0}}$. 



\section{Resumen}

El objetivo de esta tesis es desarrollar herramientas de lógica modal que puedan ser utilizadas en el campo de la teoría de la demostración y el análisis ordinal. Más precisamente, nos centramos en la relación entre las lógicas modales estrictamente positivas y las progresiones de Turing, y entre dichas lógicas y los sistemas de notación ordinal que surgen de ellas.

Con respecto a la primera parte, hemos introducido el sistema TSC, diseñado para generar exactamente todas las relaciones válidas entre las diferentes progresiones de Turing, dado un conjunto particular de nociones de consistencia naturales. También presentamos una interpretación aritmética para este sistema modal, denominada interpretación de las Progresiones de Turing formalizadas. Demostramos que la lógica es aritméticamente correcta y completa con respecto a esta interpretación.

Tras de estudiar la semántica aritmética de TSC, investigamos la semántica relacional de este sistema. Para este propósito, hacemos uso del modelo universal para el fragmento cerrado de Gödel-Löb's Polymodal Logic (GLP), a saber, el marco universal de Ignatiev. Modificando ligeramente las relaciones definidas en este modelo, obtenemos un nuevo marco. Demostramos que éste es un modelo universal para TSC. Asimismo, mostramos cómo el dominio de este marco puede reducirse a secuencias con soporte finito manteniendo la completud del sistema.

Respecto a los sistemas de notación ordinal, presentamos la lógica BC (por Bracket Calculus). A diferencia de otras lógicas de la demostrabilidad, BC se basa en un lenguaje puramente modal que da lugar a un sistema de notación ordinal, en lugar de estar construido mediante modalidades indexadas por algún ordinal dado a priori. Además, ya que el orden entre estas notaciones puede establecerse en términos de derivabilidad dentro del cálculo, las inferencias en este sistema pueden llevarse a cabo sin usar ninguna propiedad externa de los ordinales. Demostramos que la lógica presentada es equivalente al Reflection Calculus $\left(\mathbf{R C}_{\Gamma_{0}}\right)$, es decir, al fragmento estrictamente positivo de $\mathbf{G L P}_{\Gamma_{0}}$. 



\section{Introduction}

Among other interesting features, modal logic provides us with a tool to study different mathematical phenomena. Usually, a modal system presents two modal operators, $\square$ and $\diamond$, that are intended to be read as It is necessary that and It is possible that, respectively. However, there is an extensive range of interpretations for these modal operators that yields a wide family of modal systems. According to these interpretations we can speak about temporal logic, deontic logic, doxastic logic, etc.

One of the key ingredients of this dissertations is provability logic. In these modal systems the regular modalities are interpreted as provability predicates and consistency statements for arithmetical theories such as Peano Arithmetic (PA). The best known provability logic is GL (Gödel-Löb), obtained by extending the smallest normal modal logic $\mathbf{K}$ with a modal version of a formalization of Löb's theorem. This system was later generalized by Japaridze with $\mathbf{G L P}_{\omega}$; a polymodal version of GL in which we have modal operators for each $n \in \omega$, and later on extended to a class-sized logic by Beklemishev where we have modalities for each ordinal.

The main aspect of this line of research is that we can use simple tools like these modal systems to perform complex analyses of arithmetical theories. In particular, Beklemishev showed that the closed fragment of $\mathbf{G L P}_{\omega}$ was enough to perform a $\Pi_{1}^{0}$-ordinal analysis of PA and similar arithmetical theories. The present dissertation is in the context of this line of research. The aim of this work is to contribute to the field of provability logic by providing new modal systems that can be used as tools in the area of ordinal analysis.

The first goal of this investigation is related to Turing progressions. In his PhD dissertation, Alan Turing introduced what he called systems of logics based on ordinals as a way to deal with Gödel's incompleteness results. Roughly speaking, the systems of logics were obtained by iteratedly extending a formal system $L$ with one of these non-provable statements, such as the consistency of $L$. This hierarchy of theories is what we now call Turing progressions and can be defined (given an ordinal representation over which our ordinals range) by the following three clauses: 
T1. $(T)^{0}:=T$ where $T$ is an initial or base theory;

T2. $(T)^{\alpha+1}:=(T)^{\alpha} \cup\left\{\operatorname{Con}\left((T)^{\alpha}\right)\right\}$;

T3. $(T)^{\lambda}:=\bigcup_{\beta<\lambda}(T)^{\beta}$, for $\lambda$ a limit ordinal.

This construction can be extended to different consistency notions that can be naturally defined in weak fragments of arithmetic. This yields different progressions that, in turn, can be used as the base theory of a new hierarchy based on a different consistency notion, and so on. Facing this situation, it is therefore natural to ask which principles govern the interactions between these different Turing progressions. The contribution of our work with respect to this question is to provide a modal system capable of generating all possible relations that hold between different Turing progressions and where each modal formula can be used to directly denote one of these theories.

Steps in this direction were already taken from both arithmetical and provability logic perspectives. From the arithmetical point view, a cornerstone in this investigation is the fine-structure formula theorem proven by Schmerl and later generalized by Beklemishev. This result contains two conservativity principles:

$$
\begin{aligned}
& \text { S1 } \quad \forall \alpha \succ 0\left((T)_{n+m}^{\alpha} \equiv_{n}(T)_{n}^{e^{m}(\alpha)}\right) \text {; } \\
& \text { S2 } \forall \beta \succ 0\left(\left((T)_{n+m}^{\beta}\right)_{n}^{\alpha} \equiv_{n}(T)_{n}^{e^{m}(\beta) \cdot(1+\alpha)}\right) .
\end{aligned}
$$

Item $S_{1}$ is commonly known as the reduction property and states that the progression built from theory $T$ using $n+m$-consistency up to level $\alpha$ has the same $\Pi_{n+1}^{0}$-consequences as the progression obtained by iterating $n$ consistency over the same base theory up to the $\omega$-exponential tower

$$
\omega^{\omega^{\omega^{\alpha}}}
$$

of height $m$.

The system $\mathbf{G L P}_{\omega}$ provides us with a complete characterization of the $n$ consistency notions, and so, we can give an approximation to the reduction property and analogous principles by using (the arithmetical interpretation of) modal formulas in this setting (see [32]). For example, by a simple application of $S_{1}$ we know that

$$
(\mathrm{PA})_{1}^{1} \equiv_{0}(\mathrm{PA})_{0}^{\omega} .
$$

Therefore, by considering the corresponding arithmetical interpretation, we can express this result in the language of $\mathbf{G L P}_{\omega}$ by

$$
\left\{\langle 1\rangle_{\mathrm{PA}} \top\right\} \equiv_{0}\left\{\langle 0\rangle_{\mathrm{PA}}^{k+1} \top: k<\omega\right\}=\left\{\langle 0\rangle_{\mathrm{PA}} \top,\langle 0\rangle_{\mathrm{PA}}\langle 0\rangle_{\mathrm{PA}} \top, \ldots\right\} .
$$



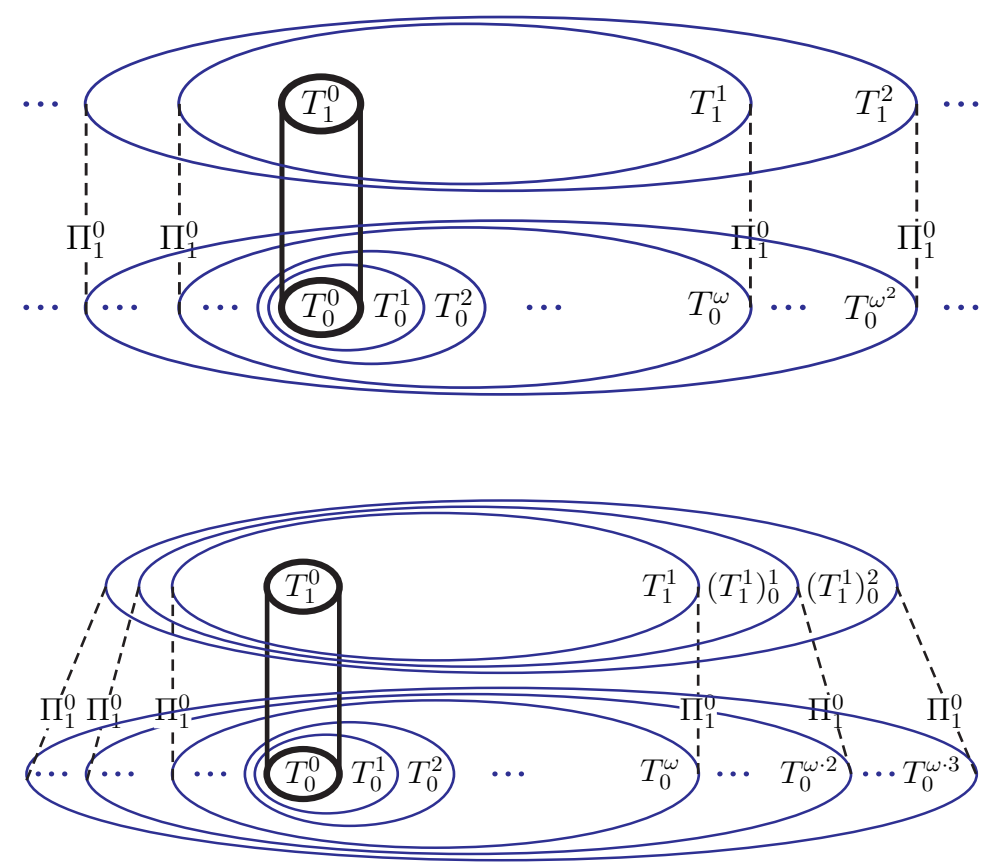

Figure 1: Graphical representations of some instances of $S_{1}$ and $S_{2}$, respectively.

While $\langle 1\rangle \top$ and each $\langle 0\rangle^{m} \top$ for $m<\omega$ are formulas in the signature of $\mathbf{G L P}_{\omega},\langle 0\rangle^{\omega} \top$ is not. Thus, we cannot directly denote (PA $)_{0}^{\omega}$ with a single modal formula but with an infinite set of them. This way, results like the fine-structure formula can be approximated by using $\mathbf{G L P}_{\omega}$ but cannot be formulated as such within this modal system.

In Chapter 2 we present a way of dealing with this issue. We introduce ordinal modalities, modal operators of the form $\left\langle n^{\alpha}\right\rangle$ where $n<\omega$ and $\alpha<\Lambda$ for $\Lambda$ a fixed recursive $\varepsilon$-number. The underlying idea of this operator is to encapsulate the iteration of the $n$-consistency notion up to level $\alpha$. Thus, our previous example can be expressed in this setting by:

$$
\left\langle 1^{1}\right\rangle_{\mathrm{PA}} \top \equiv_{0}\left\langle 0^{\omega}\right\rangle_{\mathrm{PA}} \top \text {. }
$$

Moreover, the signature we define in this chapter is a strictly positive language (with no propositional variables), that is, we only consider formulas obtained by the following grammar: $T$, conjunction and ordinal modalities. In this sense, the approach we follow is influenced by recent works papers by Dashkov and Beklemishev where they introduced the logic Reflection Calculus (RC). This system is weaker than GLP, yet expressive enough to maintain its main proof-theoretic applications. More precisely, RC con- 
sists of the fragment of GLP containing all implications of the form $\varphi \rightarrow \psi$ where $\varphi$ and $\psi$ are strictly positive formulas.

In general, by going to a strictly positive language, we gain a broader range of arithmetical interpretations. More precisely, the use of strictly positive formulas allows us to interpret modal formulas as arithmetical theories rather than individual arithmetical sentences. Therefore, in our setting, the modal operators are no longer conceived as consistency statements but theories that might be infinitely axiomatized.

With this handy signature, we also use Chapter 2 to introduce the system TSC for Turing-Schmerl Calculus. The derivable objects of this system are sequents of the form $\varphi \vdash \psi$ where $\varphi$ and $\psi$ are formulas in the above signature.

With all these ingredients, one can easily conjecture how the arithmetical semantics of this system are defined. First, we identified modal formulas with Turing progressions built by iterating different consistency notions over a weak fragment of arithmetic. In particular, we consider the conjunction of two formulas as the union of the respective theories. After this, the arithmetical interpretation of the sequents is defined as the (formalized) entailment between these theories. Thus, sequents are translated into arithmetical formulas expressing such entailment. This arithmetical interpretation is what we name the Formalized Turing progressions (FTP) interpretation. The details of this construction and the corresponding results of soundness and completeness are collected in Chapter 3.

Chapter 4 is devoted to study the relational semantics of TSC. To this end, we consider Ignatiev's universal frame for the variable-free fragment of GLP. The main drawback of GLP $\omega$ or any of its polymodal fragments is that it is incomplete with respect to its relational semantics. However, Ignatiev built a relational model for the closed fragment of the signature in which every consistent formula of variable-free $\mathbf{G L P}_{\omega}$ is satisfied [30]. This frame consists of a domain based on certain decreasing sequences of ordinals $(\ell-$ sequences) and a family of relations $\left\{T_{n}\right\}_{n<\omega}$.

In this chapter we define a modal model $\mathcal{J}$ which is universal for our logic in the sense that any derivable sequent holds everywhere in the model whereas any non-derivable sequent is refuted by some point in the frame. This new frame is defined by preserving the domain given in Ignatiev's frame but consider a slightly different family of relations $\left\{R_{n}\right\}_{n<\omega}$. Apart from this, we define the auxiliary relations $R_{n}^{\alpha}$ to naturally model the behavior of the ordinal modalities $\left\langle n^{\alpha}\right\rangle$. After defining $\mathcal{J}$ and these auxiliary relations, we use the remainder of the chapter to show the soundness and completeness results together with some useful tools about the definable sets in the frame and the characterization of the $R_{n}^{\alpha}$-relations.

In the presence of $\varepsilon$-numbers, we can define a $\ell$-sequence of the form 
$\left\langle\alpha_{0}, \alpha_{1}, \ldots, \alpha_{n}, \ldots\right\rangle$ where for each $\alpha_{i}$ we have that $\alpha_{i}>0$. On the one hand, these kind of sequences are not definable by means of any modal formula but, on the other hand, the branch of the frame where all these sequences live does not contain any additional information that is not present in any other part of the frame. Thus, by restricting our frame to sequences with finite support, that is, those such that eventually reach 0 , we obtain a new frame for which we show that TSC is complete with respect to. In this new frame, every world becomes modally definable. The relevance of this feature with respect to the main aim of TSC is that we can use the frame as a road map of Turing progressions. Thanks to the modal definability, any sequence with finite support $x$ can be translated to modal formula $\psi_{x}$ and this formula itself can be translated into Turing progressions $\mathrm{Th}_{\psi_{x}}$. Furthermore, by checking whether two sequences are related in the frame, we can derive an entailment principle between their corresponding Turing progressions. These results are collected in Chapter 5 .

In view of Gödel's second incompleteness theorem, we know that the consistency of any sufficiently powerful formal theory cannot be established using purely 'finitary' means. Since then, the field of proof theory, and more specifically of ordinal analysis, has been successful in measuring the non-finitary assumptions required to prove consistency assertions via computable ordinals. Among the benefits of this work is the ability to linearly order natural theories of arithmetic with respect to notions such as their 'consistency strength' (e.g., their $\Pi_{1}^{0}$ ordinal) or their 'computational strength' (their $\Pi_{2}^{0}$ ordinal).

Nevertheless, the assignment of these proof-theoretic ordinals to formal theories depends on a choice of a 'natural' presentation for such ordinals, with well-known pathological examples having been presented by Kreisel [33] and Beklemishev [3]. These representation systems are what we know as ordinal notation systems. During my research stay in IRIT, FernándezDuque suggested several questions regarding provability logics and the ordinal notation systems that arise from them. One of these problems is successfully solved in Chapter 6.

Some connections between ordinal notation systems and provability logics are already known and have been intensively studied (see [20], [7]). Within the closed fragment of $\mathbf{G L P}_{\omega}$, there are some special formulas that are commonly known as worms; formulas obtained by iterating different diamonds over top. Thus, worms are formulas of the form $\left\langle n_{0}\right\rangle \ldots\left\langle n_{k}\right\rangle T$. These formulas can be well ordered (modulo equivalence in $\mathbf{G L P}_{\omega}$ ) according to their consistency strength, that is, given two worms $A$ and $B$ :

$$
A<_{0} B: \Longleftrightarrow B \vdash\langle 0\rangle A
$$

Hence, it is possible to associate an ordinal $o(A)$ to any worm $A$. The set of $\mathbf{G L P}_{\omega}$-worms provide us with an ordinal notation system for $\varepsilon_{0}=$ 
$\sup \left\{\omega, \omega^{\omega}, \omega^{\omega^{\omega}}, \ldots\right\}=\varphi_{1}(0)$ which measures the precise proof-theoretical strength of PA. We can go further by considering bigger fragments of GLP in which diamonds with transfinite ordinal entries are available. Extensions of $\mathbf{G L P}_{\omega}$, denoted $\mathbf{G L P}_{\Lambda}$, have been considered in cases where $\Lambda$ is an ordinal $[7,19,24]$ or even an arbitrary linear order [12]. Proof-theoretic interpretations for $\mathbf{G L P}_{\Lambda}$ have been developed by Fernández-Duque and Joosten [25] for the case where $\Lambda$ is a computable well-order.

In such cases, we are in need of a system of notation that can represent an ordinal $\lambda<\Lambda$. Fortunately we may 'borrow' this notation from finitary worms and represent $\lambda$ itself as a worm. Iterating this process we obtain the autonomous worms, whose order types are exactly the ordinals below the Feferman-Schütte ordinal $\Gamma_{0}$, that is, the smallest fixpoint of the Veblen functions.

\begin{tabular}{|c|c|c|}
\hline() & $\langle 0\rangle \top$ & 1 \\
$(())$ & $\langle 1\rangle \top$ & $\omega$ \\
$(())(()())$ & $\langle 1\rangle\langle 2\rangle \top$ & $\omega^{\omega+1}$ \\
$((()))$ & $\langle\omega\rangle \top$ & $\varepsilon_{0}$ \\
$(())()((()))$ & $\langle 1\rangle\langle 0\rangle\langle\omega\rangle \top$ & $\varepsilon_{0}+\omega$ \\
\hline
\end{tabular}

Table 1: Some correspondences between brackets expressions, worms and ordinals.

By iterating this process we obtain a notation system for worms which uses only parentheses, as ordinals (including natural numbers) can be iteratively represented in this fashion. Thus a balanced brackets expression $a$ can be used to denote an ordinal by fist translating $a$ into a worm $A$ and then computing $o(A)$ (see Table 1 ). This is Beklemishev's brackets notation system, introduced in [7], which provides a notation system for $\Gamma_{0}$ without any reference to an externally given ordinal.

The drawback of Beklemishev's brackets system is that the actual computation of the ordering between different expressions is achieved via a translation into a traditional ordinal notation system. The goal of this investigation is to remove the need for such an intermediate step by providing an autonomous calculus for determining the ordering relation and, more generally, the logical consequence relation between bracket notations. To this end in Chapter 6 we present the Bracket Calculus (BC).

The signature of $\mathbf{B C}$ is obtained by extending the balanced brackets grammar to a strictly positive modal signature in which we use the brackets expressions as modal operators. Therefore, the set of formulas is built from $\top$ and propositional variables using conjunctions $\wedge$ and modalities of the 
form ( $a$ ) where $a$ is a balanced brackets expression. The main feature of this system is that, unlike other provability logics, BC is based on a purely modal signature that gives rise to an ordinal notation system instead of using modalities indexed by some ordinal given a priori. Moreover, since the order between these notations can be established in terms of derivability within the calculus, the inferences in this system can be carried out without using any external property of ordinals. To the best of our knowledge, this yields the first ordinal notation system presented as a purely modal deductive system. We prove that our calculus is sound and complete with respect to a natural translation into $\mathbf{R C}_{\Gamma_{0}}$.

Lastly, we would like to point out that the results contained in this dissertation have led to the following publications:

1. E. Hermo-Reyes and J. J. Joosten. The logic of Turing progressions. Accepted for publication in Notre Dame Journal of Formal Logic;

2. E. Hermo-Reyes and J. J. Joosten. Relational semantics for the Turing Schmerl calculus. In Advances in Modal Logic, volume 12, pages 327346. College Publications, 2018;

3. D. Fernndez-Duque and E. Hermo-Reyes. A self-contained provability calculus for $\Gamma_{0}$. In Logic, Language, Information, and Computation WoLLIC 2019, volume 11541 of Lecture Notes in Computer Science, pages 195-207. Springer, 2019 



\section{CHAPTER}

\section{Preliminaries}

\subsection{The Language of Arithmetic}

Throughout this dissertation $\mathcal{L}_{\mathbb{N}}$ will denote the first-order language of arithmetic, that is, a first-order language whose non-logical symbols are the following: two binary function symbols + and - for addition and multiplication; two unary function symbols $S$ and exp, for the successor function and binary exponentiation; two binary relation symbols $=$ and $\leq$, for equality and "less than or equal to"; and a constant symbol 0. The standard model of arithmetic is the $\mathcal{L}_{\mathbb{N}}$-structure whose domain is the set of natural numbers $\mathbb{N}$ and where the symbols of $\mathcal{L}_{\mathbb{N}}$ are given by their obvious interpretation.

Terms in $\mathcal{L}_{\mathbb{N}}$ are defined as usual. An occurrence of a quantifier is said to be bounded if it is of the form $\forall x \leq t \varphi(x)$ or $\exists x \leq t \varphi(x)$, where $t$ is a term not containing $x$. These expressions are abbreviations for the formulas $\forall x(x \leq t \rightarrow \varphi(x))$ and $\exists x(x \leq t \wedge \varphi(x))$, respectively. Those formulas all of whose quantifiers are bounded are called elementary formulas. The set of elementary formulas is denoted by $\Delta_{0}^{0}(\exp )$.

The following definition allows us to classify arithmetical formulas according to their logical complexity. This classification is called the arithmetical hierarchy.

Definition 1.1.1. For $n \geq 0$, the classes of $\Sigma_{n}^{0}$ - and $\Pi_{n}^{0}$-formulas are inductively defined as follows:

I) $\Sigma_{0}^{0}=\Pi_{0}^{0}=\Delta_{0}^{0}$;

II) $\Sigma_{n+1}^{0}$-formulas are of the form $\exists x_{1} \ldots \exists x_{m} \varphi\left(x_{1}, \ldots, x_{m}\right)$ where $\varphi$ is a $\Pi_{n}^{0}$ formula. Analogously, a formula is in $\Pi_{n+1}^{0}$ if it is of the form $\forall x_{1} \ldots \forall x_{m} \varphi\left(x_{1}, \ldots, x_{m}\right)$ where $\varphi$ is a $\Sigma_{n}^{0}$-formula. 
Notice that, by the prenex normal form theorem, every formula is equivalent to a formula of the form $\mathrm{Q} x_{1} \ldots \mathrm{Q} x_{m} \varphi\left(x_{1}, \ldots, x_{m}\right)$ where $\varphi$ is quantifierfree, and so an elementary formula, and $\mathrm{Q}$ is either $\forall$ or $\exists$ for each $i$. Therefore, every formula is equivalent to some $\Sigma_{n}^{0}$ - or $\Pi_{n}^{0}$-formula for some $n$. Naturally, theses equivalences take place in some fixed theory $T$. Usually, $T$ will be clear from the context. We shall sometimes call $\Sigma_{n}^{0}\left(\Pi_{n}^{0}\right)$ any formula that is logically equivalent to a $\Sigma_{n}^{0}$-formula ( $\Pi_{n}^{0}$-formula).

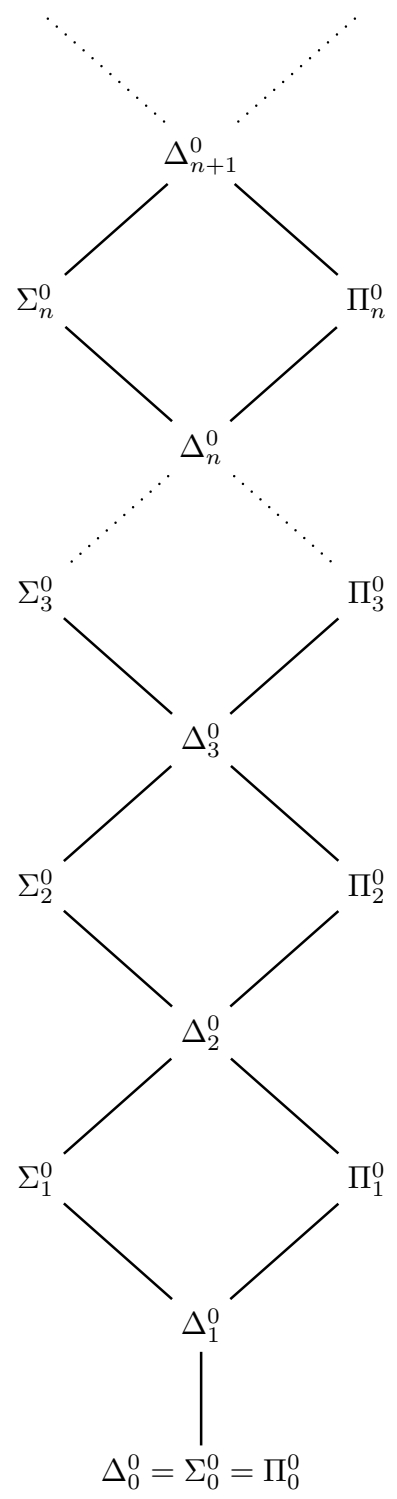

Figure 1.1: The arithmetical hierarchy.

We say that a formula is $\Delta_{n}^{0}$ iff it is equivalent to both a $\Sigma_{n}^{0}$-formula and a $\Pi_{n}^{0}$-formula. As before, the theory $T$ where these equivalences occur 
will be clear. Since we might have empty blocks of quantifiers in the above definition, we obtain some obvious inclusions between these classes: $\Sigma_{n}^{0} \subseteq$ $\Delta_{n+1}^{0} \subseteq \Sigma_{n+1}^{0}$ and $\Pi_{n}^{0} \subseteq \Delta_{n+1}^{0} \subseteq \Pi_{n+1}^{0}$ (see Figure 1.1). Moreover, we have that $\Sigma_{n}^{0} \cup \Pi_{n}^{0} \subseteq \Delta_{n+1}^{0} \subseteq \Sigma_{n+1}^{0} \cap \Pi_{n+1}^{0}$.

The classes $\Sigma_{n}^{0}$ and $\Pi_{n}^{0}$ are closed under $\wedge$ and $\vee$. With respect to negation, if $\varphi(x)$ is a $\Sigma_{n}^{0}$-formula, $\neg \varphi(x)$ is equivalent (in the predicate calculus) to a $\Pi_{n}^{0}$-formula and vice-versa, $\neg \varphi(x)$ with $\varphi(x) \in \Pi_{n}^{0}$ is equivalent to a $\Sigma_{n}^{0}$-formula. Hence, $\Delta_{n}^{0}$-formulas are closed under $\wedge$ and $\vee$, but also under $\neg$.

It is worth mentioning that, from a computational perspective, the bottom of the hierarchy is particularly relevant. A set on $\mathbb{N}$ is definable by a $\Sigma_{1}^{0}$-formula iff it is recursively enumerable (r.e.). Thus, since the negation of a $\Sigma_{1}^{0}$-formula is equivalent to a $\Pi_{1}^{0}$ formula, the $\Pi_{1}^{0}$ definable sets are the co-r.e. sets. The $\Delta_{1}^{0}$ sets correspond exactly to the recursive sets (rec), and so, any $\Delta_{0}^{0}$ definable set is decidable. Moreover, every $\Delta_{0}^{0}$ definable set is primitive recursive (p.r.). In Figure 1.2 we can find a diagram of the bottom of the arithmetical hierarchy from a computational point of view.

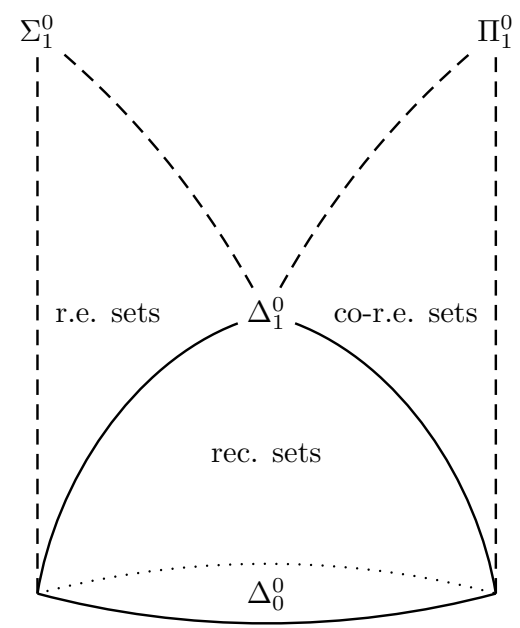

Figure 1.2: The bottom of the arithmetical hierarchy from a computational point of view.

\section{Arithmetical theories}

The need for a formal development of arithmetic was outlined during the second half of the nineteenth century in the work of Hermann Grassmann, Charles Sanders Peirce and Richard Dedekind among others. However, it was not until 1889, with the publication of Arithmetices principia, nova methodo exposita by Giuseppe Peano, that formal arithmetic began to take its current shape. 
In his seminal work, Peano introduced his collection of principles for natural-number arithmetic that we nowadays know as Peano Arithmetic PA. In this subsection we shall introduce the axioms of PA together with some other weaker theories, all of them formulated in the arithmetical language $\mathcal{L}_{\mathbb{N}}$.

By PA we denote the theory containing all classical axioms of first order logic along with the following mathematical axioms:

P1. $\neg S(a)=0$;

P2. $S(a)=S(b) \rightarrow a=b$;

P3. $a+0=a$;

P4. $a+S(b)=S(a+b)$;

$\mathrm{P}_{5} \cdot a \cdot 0=0$;

P6. $a \cdot S(b)=a \cdot b+a$;

P7. $\exp (0)=S(0)$;

P8. $\exp (S(a))=\exp (a)+\exp (a)$;

P9. $a \leq 0 \leftrightarrow a=0$;

P10. $a \leq S(b) \leftrightarrow(a \leq b \vee a=S(b))$.

Apart from these axioms, PA also contains the induction axiom for all arithmetical formulas $\varphi(x)$ :

$$
\mathrm{I}_{\varphi}: \quad \varphi(0) \wedge \forall x(\varphi(x) \rightarrow \varphi(S(x))) \rightarrow \forall x \varphi(x) .
$$

By restricting the induction scheme we can obtain different fragments of PA. Of special interest is Elementary Arithmetic EA. This fragment of PA is obtained by restricting the induction axiom to $\Delta_{0}^{0}$-formulas and considering the axioms P1 to P10.

A natural extension of EA is $\mathrm{EA}^{+}$. This theory can be obtained by constructing a predicate Supexp which represents the graph of the superexponential function $(x, y) \mapsto 2_{y}^{x}$ defined by $2_{0}^{x}:=x$ and $2_{y+1}^{x}:=2^{2_{y}^{x}}$. Therefore, we will not introduce the function symbol but only work with the predicate. We define $\mathrm{EA}^{+}$as EA + SUP-EXP where SUP-EXP is the axiom that states the totality of the super-exponential function using the predicate Supexp, that is, Sup-EXP $:=\forall x y \exists z \operatorname{Supexp}(x, y, z)$.

We can consider an equivalent formulation with a lower complexity by extending our arithmetical language by a function symbol supexp and thus, extending EA by adding to the set of axioms the corresponding recursive 
defining equations for this super-exponential function. The totality of superexponentiation can be easily proven by using induction for $\Delta_{0}^{0}$-formulas. This way, we obtain a $\Pi_{1}^{0}$-conservative extension of $\mathrm{EA}^{+}$(see [32]). However, in this case, the notion of $\Delta_{0}$ - presentable would not coincide with the more standard one from the literature.

The standard arithmetization of syntax of first order theories can be performed in $\mathrm{EA}^{+}$. Hence, arithmetical formulas can be one-to-one encoded by numbers. Given an expression $\epsilon$, we refer to such a code as the Gödel number of $\epsilon$, that will be denoted by $\ulcorner\epsilon\urcorner$. Different natural syntactical relations can be elementarily defined by using this encoding: we can code finite sequences of numbers and many of their basic properties like the length of the sequence, the $i$-st element of the sequence, concatenation, etc. Furthermore, we can also code properties about arithmetical formulas like being an arithmetical sentence, being a $\Pi_{n}^{0}$-formula, being a logical axiom or that a certain formula can be obtained by the rules of generalization or modus ponens. All this machinery combined provide us with two important applications. First, with the help of the arithmetization of syntax, theories can be represented within weak fragments of arithmetic by elementary formulas numbering their axioms. These theories are the so-called elementarily presented theories:

Definition 1.1.2 (Elementarily presented theories). A theory $U$ is elementarily presented iff there is a bounded arithmetical formula $\sigma_{U}(x)$ that defines the sets of Gödel numbers coding the axioms of $U$ in the standard model of arithmetic. Given elementary presented theories $T$ and $U$, by $T+U$ we denote the theory whose set of axioms is defined by the formula $\sigma_{T}(x) \vee \sigma_{U}(x)$. By $\epsilon(x)$ we denote the canonical presentation of $\mathrm{EA}^{+}$.

$\mathrm{EA}^{+}$and its extensions will be important throughout this thesis. However, we shall focus on extensions we can discuss from our base theory. Thus, by a theory we shall mean an elementary presented first order theory (with equality), formulated in $\mathcal{L}_{\mathbb{N}}$ and containing $\mathrm{EA}^{+}$. Via Craig's trick, any r.e. theory has an equivalent elementary presentation.

Another feature of the arithmetization of syntax is that allows us to define the predicate $\operatorname{Prf}_{T}(x, y)$ meaning " $x$ codes a proof of a formula $y$ in the theory $T$ ". Moreover, this predicate can be expressed by an elementary formula.

Gödel's provability predicate $\square_{T}(x)$ is defined as $\exists y \operatorname{Prf}_{T}(y, x)$; an arithmetical $\Sigma_{1}^{0}$-formula expressing that there is $x$ such that $x$ codes a proof in $T$ of a formula with code $y$. By $\bar{n}$ we denote the numeral $S(S(\ldots S(0) \ldots))(n$ times). Often, we write $\square_{T}(\varphi(\dot{x}))$ instead of $\square_{T}(\ulcorner\varphi(\dot{x})\urcorner)$, where $\left\ulcorner\varphi\left(\dot{x}_{1}, \ldots, \dot{x}_{j}\right)\right\urcorner$ denotes the map sending $n_{1}, \ldots, n_{k}$ to the Gödel number $\left\ulcorner\varphi\left(\overline{n_{1}}, \ldots, \overline{n_{j}}\right)\right\urcorner$. 
The following proposition collects Löb's derivability conditions that provides an analysis of the basic properties of the provability predicate:

Proposition 1.1.3 (Löb's derivability conditions). For any sentences $\varphi, \psi$ and theory T:

L1. $T \vdash \varphi \Longrightarrow \mathrm{EA} \vdash \square_{T} \varphi$;

L2. $\mathrm{EA} \vdash \square_{T}(\varphi \rightarrow \psi) \rightarrow\left(\square_{T} \varphi \rightarrow \square_{T} \psi\right)$;

L3. EA $\vdash \square_{T} \varphi \rightarrow \square_{T} \square_{T} \varphi$.

Analogous conditions hold for formulas with free variables:

$\mathrm{L}^{\prime} 1 . T \vdash \varphi(x) \Longrightarrow \mathrm{EA} \vdash \square_{T} \varphi(\dot{x}) ;$

$\mathrm{L}^{\prime}$ 2. $\mathrm{EA} \vdash \square_{T}(\varphi(\dot{x}) \rightarrow \psi(\dot{x})) \rightarrow\left(\square_{T} \varphi(\dot{x}) \rightarrow \square_{T} \psi(\dot{x})\right) ;$

$\mathrm{L}^{\prime} 3 . \mathrm{EA} \vdash \square_{T} \varphi(\dot{x}) \rightarrow \square_{T} \square_{T} \varphi(\dot{x})$;

$\mathrm{L}^{\prime} 4 . \mathrm{EA} \vdash \square_{T} \forall x \varphi(x) \rightarrow \forall x \square_{T} \varphi(\dot{x})$.

The following generalization of Gödel's famous second incompleteness theorem is known as Löb's theorem.

Theorem 1.1.4 (Löb's theorem). For any elementarily presented theory $T$ containing EA and any sentence $\varphi$ :

$$
T \vdash \square_{T} \varphi \rightarrow \varphi \Longleftrightarrow T \vdash \varphi .
$$

Moreover, Löb's theorem can be formalized within EA. This formalized version can be inferred from the non-formalized version:

Corollary 1.1.5 (Formalized Löb's theorem). For any sentence $\varphi$ :

$$
\mathrm{EA} \vdash \square_{T}\left(\square_{T} \varphi \rightarrow \varphi\right) \rightarrow \square_{T} \varphi .
$$

Proof. Let $\chi:=\square_{T}\left(\square_{T} \varphi \rightarrow \varphi\right) \rightarrow \square_{T} \varphi$. We reason as follows:

$$
\begin{aligned}
& \mathrm{EA} \\
& \quad \vdash\left(\square_{T} \chi \wedge \square_{T}\left(\square_{T} \varphi \rightarrow \varphi\right)\right) \rightarrow \square_{T} \square_{T}\left(\square_{T} \varphi \rightarrow \varphi\right) ; \\
& \quad \vdash\left(\square_{T} \chi \wedge \square_{T}\left(\square_{T} \varphi \rightarrow \square_{T}\left(\square_{T} \varphi \rightarrow \varphi\right)\right) \rightarrow\left(\square_{T} \square_{T} \varphi \wedge\left(\square_{T} \square_{T} \varphi \rightarrow \square_{T} \varphi\right)\right) ;\right.
\end{aligned}
$$

Thus, we have that

$$
\mathrm{EA} \vdash \square_{T} \chi \rightarrow \chi
$$

By monotonicity we get that $T \vdash \square_{T} \chi \rightarrow \chi$. Hence, by Löb's theorem $T \vdash \chi$ and so, EA $\vdash \square_{T} \chi$. Thus, EA $\vdash \chi$. 
By $\operatorname{Con}(T)$ we denote the formula expressing the consistency of the theory $T$. This formula can be defined as $\operatorname{Con}(T):=\neg \square_{T} 0=1$ or equivalently, $\operatorname{Con}(T):=\square_{T} 0=1 \rightarrow 0=1$. Therefore, by substituting $0=1$ for $\varphi$ in Löb's theorem, we obtain Gödel's second incompleteness theorem as a corollary (see [26]).

Theorem 1.1.6 (Gödel's second incompleteness theorem). For any elementarily presented theory $T$ containing EA:

$$
\text { If } T \text { is consistent, then } T \nvdash \operatorname{Con}(T) \text {. }
$$

Moreover, if, in addition, $T$ is $\Sigma_{1}^{0}$-sound i.e. all $\Sigma_{1}^{0}$ theorems are true in the standard model of arithmetic, then $T \forall \neg \operatorname{Con}(T)$.

Up to this point, we have seen that provability and consistency have their own predicates that can be formalized within EA. This is not the case when it comes to truth. By the Gödel-Tarski undefinability theorem we know that there is no formula $\operatorname{Tr}(x)$ such that for all sentences $\varphi$ we have that:

$$
\mathrm{EA} \vdash \varphi \leftrightarrow \operatorname{Tr}(\varphi)
$$

However, it is well known that for the classes $\Sigma_{n}^{0}$ and $\Pi_{n}^{0}$-formulas we can provide truth-definitions within EA.

Proposition 1.1.7 (Partial truth predicates). For any $n<\omega$, there is an arithmetical $\Sigma_{n}^{0}$-formula $\operatorname{Tr}_{\Sigma_{n}^{0}}(x)$ such that for all $\Sigma_{n}^{0}$-formulas $\varphi\left(x_{1}, \ldots, x_{n}\right)$ :

$$
\mathrm{EA} \vdash \varphi\left(x_{1}, \ldots, x_{n}\right) \leftrightarrow \operatorname{Tr}_{\Sigma_{n}^{0}}\left(\varphi\left(\dot{x}_{1}, \ldots, \dot{x_{n}}\right)\right) .
$$

The analogous result holds for $\Pi_{n}^{0}$-formulas.

With this partial truth definitions, we can talk about $n$-consistency and so, about $n$-provability. For $n \geq 1$, by $\operatorname{Tr}_{\Pi_{n}^{0}}(\mathbb{N})$ we denote the set of all true arithmetical $\Pi_{n}^{0}$-sentences. For any arithmetical theory $T$, we shall say that $T$ is $n$-consistent iff $T+\operatorname{Tr}_{\Pi_{n}^{0}}(\mathbb{N})$ is consistent. Furthermore, this notion can be formalized within EA by the following $\Pi_{n+1}^{0}$-formula:

$$
\operatorname{CoN}_{n}(T):=\forall x\left(\operatorname{Tr}_{\Pi_{n}^{0}}(x) \rightarrow \neg \square_{T} \neg \operatorname{Tr}_{\Pi_{n}^{0}}(x)\right) .
$$

The $n$-consistency of the theory $T$ can equivalently (in EA) be expressed (see e.g. [6]) by the arithmetical formula:

$$
\operatorname{Con}_{n}(T):=\forall x \in \Pi_{n+1}^{0}\left(\square_{T}(x) \rightarrow \operatorname{Tr}_{\Pi_{n+1}^{0}}(x)\right)
$$

where $x \in \Pi_{n+1}^{0}$ expresses that $x$ is the Gödel number of a $\Pi_{n+1}^{0}$ sentence. 
The dual n-provability formula:

$$
[n]_{T} \varphi:=\neg \operatorname{Con}_{n}(T+\neg \varphi)
$$

expresses the provability of $\varphi$ in $T$ with the help of all true $\Pi_{n}^{0}$-formulas. For these new provability predicates we have the analogus properties of the usual one. Thus, we have Löb's conditions and Löb's (formalized) theorem for $[n]_{T}$ (see [6]). As we shall see, the notion of $n$-consistency will be the key to graded Turing progressions and to introduce the motivation for the Gödel-Löb's polymodal logic (GLP).

\subsection{Ordinals}

In this section we shall introduce some basic features about ordinal numbers which are one of the key elements of this dissertation. Ordinal numbers are canonical representatives of well-orders. We can see ordinals as a generalization of the natural numbers into the transfinite, used to describe ordered collections of objects. In this sense, we can say that ordinal numbers measure the length of well-ordered sets.

Let us start by recalling the notion of well-order. Given a set $X$, a preorder on $X$ is a reflexive and transitive relation on $X$. Moreover, a linear order is an antisymmetric and total preorder. A total preorder where every non-empty $Y \subseteq X$ has a minimal element is a pre-well-order. We say that $\langle X, \preccurlyeq\rangle$ is a well-order if $\preccurlyeq$ is a linear pre-well-order.

The following definition introduces ordinal numbers as a particular case of a well-ordered set.

Definition 1.2.1. We say that a set $A$ is transitive if whenever $B \in A$, it follows that $B \subseteq A$. Then, a set $\xi$ is an ordinal if $\xi$ is transitive and $\langle\xi, \in\rangle$ is a well-order.

From now on, we shall adopt the following notation: by Ord we denote the class of ordinal numbers. Furthermore, given ordinals $\alpha, \beta$, we shall equally use $\alpha<\beta$ for $\alpha \in \beta$ and $\alpha \leq \beta$ if $\alpha<\beta$ or $\alpha=\beta$.

The class of all ordinals, Ord, is well-ordered by $\in$. Thus, for any ordinal $\alpha$, we have that $\alpha=\{\beta \in$ Ord $: \beta<\xi\}$, and $0=\varnothing$ is the least ordinal. There are two basic operations that we can define on Ord. For $\alpha \in \operatorname{Ord}$ by $\alpha+1$ we denote $\alpha \cup\{\alpha\}$; that is the least ordinal greater than $\alpha$. Therefore, starting by 0 and iterating this operation, we can observe that any natural number is an ordinal. Moreover, new ordinals can be formed by taking unions. Hence, given $\Theta$ a set of ordinals, we have that $\lambda=\bigcup \Theta$ is an ordinal as well. Thus, we also have infinite ordinals; the union of the set of natural numbers is 
itself an ordinal greater that each natural number. We denote this ordinal by $\omega$.

The following lemma summarizes these observations:

Lemma 1.2.2. For any $\alpha \in$ Ord exactly one of the following occurs:

1. $\alpha=0$;

2. there exists $\beta$ such that $\alpha=\beta+1$, in which case we say that $\alpha$ is a successor; or

3. $\alpha=\bigcup_{\beta<\alpha} \beta$, in which case we say that $\alpha$ is a limit.

By Succ we shall denote the class of successor ordinals and analogously, Lim denotes the class of limit ordinals.

This generalization of the natural numbers also extends to the induction schema. Transfinite induction is an extension of regular mathematical induction to well-ordered sets, and so, it can be applied to ordinal numbers.

Definition 1.2.3 (Transfinite induction). Let $P$ be a property and $X$ a wellordered set. We define the transfinite induction axiom for $P$ by

$$
\forall \alpha \in X(\forall \beta<\alpha P(\beta) \rightarrow P(\alpha)) \quad \Longrightarrow \quad \forall \alpha \in X P(\alpha)
$$

It is worth mentioning that the principle of transfinite induction also holds when considering the class Ord instead of $X$.

\section{Order-types}

As we have mentioned, a well-order $\mathfrak{X}=\langle X, \preccurlyeq\rangle$ can be canonically represented by using an ordinal. To do this, we define $o: X \rightarrow$ Ord such that:

$$
o(x)=\bigcup_{y \prec x}(o(y)+1) .
$$

We say that $o(x)$ is order-type of $x$. The rank of $\mathfrak{X}$ is then defined as $\sup _{x \in X}(o(x)+$ 1).

The following lemma is useful in characterizing the rank function [20].

Lemma 1.2.4. Let $\langle X, \preccurlyeq\rangle$ be a well-order. Then $o: X \rightarrow$ Ord is the unique function such that

1. $x \prec y$ implies that $o(x)<o(y)$,

2. if $\xi<o(x)$ then $\xi=o(y)$ for some $y \in X$. 


\section{Ordinal arithmetic}

In the previous section we showed how to define the successor of any given ordinal $\alpha$. In this section we shall see how we can define the basic arithmetic operations and outline some of their properties.

We shall start defining the addition function for ordinals. We do this by transfinite recursion. As we can observe, this definition is analogous to the one for the natural numbers, with the exception that now we consider an extra step for limit ordinals.

Definition 1.2.5 (Ordinal addition). Given ordinals $\alpha, \beta$, we define $\alpha+\beta$ by recursion on $\beta$ as follows:

1. $\alpha+0=\alpha$;

2. $\alpha+(\beta+1)=(\alpha+\beta)+1$;

3. $\alpha+\beta=\bigcup_{\delta<\beta}(\alpha+\delta)$, for $\beta \in$ Lim.

In a similar fashion we can define the product $(\alpha \cdot \beta)$ and the exponentiation $\left(\alpha^{\beta}\right)$.

The following lemma collects some observations about these arithmetic operations.

Lemma 1.2.6. For any $\alpha, \beta, \delta \in$ Ord:

1. $(\alpha+\beta)+\delta=\alpha+(\beta+\delta)$;

2. if $\alpha+\beta=\alpha+\delta$, then $\beta=\delta$;

3. $\alpha \cdot(\beta+\delta)=\alpha \cdot \beta+\alpha \cdot \delta$;

4. $\alpha^{\beta} \cdot \alpha^{\delta}=\alpha^{(\beta+\delta)}$;

5. $\left(\alpha^{\beta}\right)^{\delta}=\alpha^{\beta \cdot \delta}$.

As a particular case of the exponentiation function, we have the mapping $\alpha \mapsto \omega^{\alpha}$. This function is the basis of the Cantor normal form representation of ordinals:

Lemma 1.2.7 (Cantor normal form). For any $\alpha \in$ Ord with $\alpha>0$, there are $\alpha_{0} \geq \alpha_{1} \geq \ldots \geq \alpha_{k}$ for some $k<\omega$ such that:

$$
\alpha=\omega^{\alpha_{0}}+\ldots \omega^{\alpha_{k}} .
$$

A non-zero ordinal $\alpha$ such that for any $\delta, \gamma<\alpha$ we have that $\delta+\gamma<\alpha$ is called an additively indecomposable ordinal. These ordinals are exactly the ordinals of the form $\omega^{\beta}$ for some $\beta$. 


\section{Normal functions and hyper-exponentiation}

The arithmetic operations defined in the previous section are examples of normal functions, that is, functions $f:$ Ord $\rightarrow$ Ord which are strictly increasing and continuous, in the sense that if $\lambda$ is a limit then $f(\lambda)=\sup _{\alpha<\lambda} f(\alpha)$. This notion can be formalized as follows:

Definition 1.2.8. A function $f:$ Ord $\rightarrow$ Ord is normal iff:

1. $f(\alpha)<f(\beta)$ whenever $\alpha<\beta$, and

2. $f(\lambda)=\sup _{\alpha<\lambda} f(\alpha)$ for $\lambda \in \operatorname{Lim}$.

When $f: X \rightarrow X$, it is natural and often useful to ask whether $f$ has fixed points, i.e., solutions to the equation $x=f(x)$. In particular, normal functions have many fixed points:

Proposition 1.2.9. Every normal function on Ord has arbitrarily large fixed points.

The first ordinal $\alpha$ such that $\alpha=\omega^{\alpha}$ is the limit of the $\omega$-sequence

$$
\left\langle\omega, \omega^{\omega}, \omega^{\omega^{\omega}}, \ldots\right\rangle,
$$

and is usually denoted $\varepsilon_{0}$. Every $\xi<\varepsilon_{0}$ can be written in terms of 0 using only addition and the function $\omega \mapsto \omega^{\xi}$ via its Cantor normal form. The hyper-exponential function is then a natural transfinite iteration of the ordinal exponential which remains normal after each iteration.

Definition 1.2.10 (Hyper-exponential functions). The hyper-exponential functions $\left(e^{\zeta}\right)_{\zeta \in \text { Ord }}$ are the unique family of normal functions that satisfy

1. $e^{1}=e$,

2. $e^{\alpha+\beta}=e^{\alpha} \circ e^{\beta}$ for all $\alpha$ and $\beta$, and

3. if $\left(f^{\xi}\right)_{\xi \in \text { Ord }}$ is a family of functions satisfying 1 and 2 , then for all $\alpha, \beta \in$ Ord, $e^{\alpha} \beta \leq f^{\alpha} \beta$.

Fernández-Duque and Joosten proved that the hyperexponentials are well-defined [22]. In [20] it is also shown that the function $\xi \mapsto e^{\xi} 1$ is itself a normal function, hence it has a least non-zero fixed point: this fixed point is the Feferman-Schütte ordinal, $\Gamma_{0}$. Just like ordinals below $\varepsilon_{0}$ may be written using 0 , addition, and $\omega$-exponentiation, every ordinal below $\Gamma_{0}$ may be written in terms of 0,1 , addition and the function $(\xi, \zeta) \mapsto e^{\xi} \zeta$.

As the reader might notice, there is a close link between hyper-exponential functions and the more standard Veblen functions. Notations using hyperexponentials or Veblen functions can be translated from one to the other with the help of the following proposition that can be found in [20]: 
Proposition 1.2.11. Given ordinals $\alpha, \beta$ :

1. $e^{\alpha}(0)=0$,

2. $e^{1}(1+\beta)=\varphi_{0}(1+\beta)$,

3. $e^{\omega^{1+\alpha}}(1+\beta)=\varphi_{1+\alpha}(\beta)$.

\section{Ordinal notation systems}

Broadly speaking, we can define an ordinal notation system for $\Lambda$ as a way of encoding ordinals by using finite sequences of an alphabet $A$. For this presentation, we shall focus on representing ordinals as relations defined over $\mathbb{N}$. Furthermore, we are interested in ordinal notation systems with a low arithmetical complexity.

We say $\Lambda \in$ Ord is a recursive ordinal if there is $\mathfrak{D}:=\langle D, \prec\rangle$ such that both $D \subseteq \mathbb{N}$ and $\prec$ are recursive, $\prec$ is a well-order in $D$ and the rank of $\mathfrak{D}$ is $\Lambda$. Along this thesis, we shall focus on recursive ordinals within EA ${ }^{+}$. To this purpose, we shall consider elementarily definable relations on $\mathbb{N}$. An elementary linear ordering is a structure $\mathfrak{D}:=\langle D, \prec\rangle$ where both, $D$ and $\prec$ are elementarily definable, $D \subseteq \mathbb{N}$ and $\mathrm{EA}^{+}$proves that $\prec$ linearly orders $D$. An elementary well-ordering is an elementary linear ordering that is well-founded in the standard model.

For the remainder of this dissertation we shall furthermore assume that our elementary orders are nice in that the basic properties of and operations on the elements of the ordering are available in $\mathrm{EA}^{+}$. As such we can view the elements as ordinals, we can distinguish successor ordinals from limit ordinals and perform the basic operations like addition, multiplication and exponentiation.

\subsection{Turing progressions}

The well-known theorem of Gödel shows that every system of logic is in a certain sense incomplete, but at the same time it indicates means whereby from a system $L$ of logic a more complete system $L^{\prime}$ may be obtained. By repeating the process we get a sequence $L, L_{1}=L^{\prime}, L_{2}=L_{1}^{\prime}, \ldots$ each more complete than the preceding. A logic $L_{\omega}$ may then be constructed in which the provable theorems are the totality of theorems provable with the help of the logics $L, L_{1}, L_{2}, \ldots$. We may then form $L_{2} \omega$ related to $L_{\omega}$ the same may as $L_{\omega}$ was related to $L$. Proceeding in this way we can associate a system of logic with any constructive ordinal. It may be asked whether such a sequence of logics of this kind is complete in the sense that to any problem $A$ there corresponds an ordinal $\alpha$ such that $A$ is solvable by means of the logic $L_{\alpha}$. 
After the impact of Gödel's incompleteness results, Alan Turing took the first endeavor to overcome this situation. This attempt was the topic of his PhD dissertation, published in 1939 under the title Systems of logics based on ordinals [36].

The method proposed by Turing to try to deal with Gödel's incompleteness is based on the natural idea of extending formal systems by iteratedly adding these non-provable statements, such as the consistency of the system. Furthermore, this construction can be carried out up to transfinite levels, being that given a sequence $\left\langle L^{n}\right\rangle_{n \in \mathbb{N}}$ of formal systems, we can consider a new system $L^{\omega}:=\bigcup_{n \in \mathbb{N}} L_{n}$ by just taking the union of their axioms. Then this iteration can be continued to $L^{\omega+1}$ as we did for the construction of the $L_{n}$ 's and so on. This process suggest a way of associating a formal system $L^{\alpha}$ to any recursive ordinal $\alpha$.

In particular, if we start this construction by taking as our base formal system any sound r.e. arithmetical theory $T$ that is sufficiently rich, we can build the successor step $T^{\alpha+1}$ by taking $T^{\alpha}$ together with the consistency statement for $T^{\alpha}$, Con $\left(T^{\alpha}\right)$ and therefore, by Gödel's second incompleteness theorem, we obtain a hierarchy of theories of stronger strength. These hierarchies are known as Turing progressions which nowadays are widely used in proof theory and can serve the purpose of gauging the proof-theoretic strength of mathematical theories that contain or interpret arithmetic. (See e.g. [5].)

More formally, let us assume that we have fixed some recursive limit ordinal $\Lambda$ together with a natural ordinal notation system for $\Lambda$ that is elementary presented. Turing progressions are essentially defined by the following three clauses.

T1. $(T)_{n}^{0}:=T$ where $T$ is an initial or base theory;

T2. $(T)_{n}^{\alpha+1}:=(T)_{n}^{\alpha} \cup\left\{\operatorname{Con}_{n}\left((T)_{n}^{\alpha}\right)\right\}$;

$\mathrm{T}_{3}$. $(T)_{n}^{\lambda}:=\bigcup_{\beta<\lambda}(T)_{n}^{\beta}$, for $\lambda$ a limit ordinal not exceeding $\Lambda$.

There are various ways one can define/present a series of theories that satisfy these three clauses. Here we will consider smooth Turing progressions, studied by Beklemishev in among others [6], [] and [1]. We give a slightly different presentation.

Suppose we are given some elementary well-ordering $(D, \prec)$ and an initial theory $T$. The conditions $\mathrm{T}_{1}-\mathrm{T}_{3}$ can be reformulated by the following unique clause:

$$
(T)_{n}^{\alpha}:=T \cup\left\{\operatorname{Con}_{n}\left((T)_{n}^{\beta}\right): \prec(\beta, \alpha), \beta \in D\right\} \quad \forall \alpha \in D, n<\omega .
$$

Note that there appears to be a circularity in this definition. Of course, by means of the fixed point theorem (see [16]) we can find a formalization of the definition within arithmetic. 
We say that $\tau_{n}^{\sigma(z)}(\alpha, x)$ enumerates the $\alpha$-th theory of a progression based on iteration of $n$-consistency along $(D, \prec)$ with base $\sigma(z)$ if:

$$
\begin{aligned}
\mathrm{EA}^{+} \vdash \tau_{n}^{\sigma(z)}(\alpha, x) \leftrightarrow( & (\epsilon(x) \vee \sigma(x)) \vee \\
& \left.\exists \beta\left(\prec(\beta, \alpha) \wedge x=\left\ulcorner\operatorname{Con}_{n}\left(\tau_{n}^{\sigma(z)}(\dot{\beta}, y)\right)\right\urcorner\right)\right) .
\end{aligned}
$$

The free variables of $\tau_{n}^{\sigma(z)}(\alpha, x)$ are $x$ and $\alpha$, where $x$ is the Gödel number of a formula and $\alpha$ is the code of an ordinal. Recall that by our reading conventions we have that $\operatorname{Con}_{n}\left(\tau_{n}^{\sigma(z)}(\dot{\beta}, y)\right)$ denotes the $n$-consistency of the theory axiomatized by $\tau_{n}^{\sigma(z)}(\dot{\beta}, y)$. Since the equivalence does not directly refer to $\tau_{n}^{\sigma(z)}(\dot{\beta}, y)$ but rather to the Gödel number of its $n$-consistency (that is $\left.\operatorname{Con}_{n}\left(\tau_{n}^{\sigma(z)}(\dot{\beta}, y)\right)\right)$, the existence of such $\tau_{n}^{\sigma(z)}(\alpha, x)$ is guaranteed by the fixed point theorem. Furthermore, in [2] and [4] it is shown that given an elementary presented theory $T$ and an elementary well-ordering $(D, \prec)$, there is a unique progression $(T)_{n}^{\alpha}$ for $n<\omega$ and $\alpha \in D$, modulo provable equivalence in $\mathrm{EA}^{+}$. The proof of this result makes use of a trick coming from the work of U. Schmerl [34], namely reflexive induction or sometimes called reflexive transfinite induction.

Proposition 1.3.1 (Reflexive induction). For any p.r. well-ordering $(D, \prec)$, any theory $T$ containing $\mathrm{EA}^{+}$is closed under the following reflexive induction rule:

$$
T \vdash \forall \alpha\left(\square_{T}(\forall \beta \prec \dot{\alpha} \varphi(\beta)) \rightarrow \varphi(\alpha)\right) \Longrightarrow T \vdash \forall \alpha \varphi(\alpha) .
$$

Reflexive induction is our main tool for proving facts about Turing progressions. It may seem very tricky but it has a very simple proof and is in a sense a direct consequence of Löb's theorem. Whenever we say that we are going to prove that $T$ proves $\forall \alpha \varphi(\alpha)$ by reflexive induction, we implicitly say that we are going to show that in $T$ we can prove

$$
\forall \alpha\left(\square_{T}(\forall \beta \prec \dot{\alpha} \varphi(\beta)) \rightarrow \varphi(\alpha)\right) .
$$

Further, we call the challenge of proving $T \vdash \varphi(0)$ the base case, and for given $\alpha$, refer to the assumption in $T$ that $\square_{T}(\forall \beta \prec \dot{\alpha} \varphi(\beta))$, as the reflexive induction hypothesis (RIH).

\subsection{Provability Logic}

Provability logics are modal systems in which the modal operators ( $\square$ and $\diamond)$ are interpreted as provability predicates and consistency statements for theories such as EA, EA ${ }^{+}$or PA. The basic provability logic GL (GödelLöb) is given in the language of propositional modal logic whose formulas 
are constructed from propositional variables, standard Boolean connectives and a unary modal operator $\square$. GL is a normal modal logic, that is, the theorems of GL contains all boolean tautologies and formulas of the form $\square(\varphi \rightarrow \psi) \rightarrow(\square \varphi \rightarrow \square \psi)$. Moreover, the theorems of GL are closed under substitution and the inference rules of modus ponens and necessitation. The smallest logic satisfying these conditions is the modal logic K. GL extends $\mathbf{K}$ by adding the following axiom scheme:

$$
\text { (GL) } \quad \square(\square \varphi \rightarrow \varphi) \rightarrow \square \varphi .
$$

We can observe that this axiom scheme is a modal version of the formalization of Löbs theorem as expressed in Corollary 1.1.5, which makes GL a suitable candidate to capture the behavior of the provability predicate. To show that this is the case, we need to define a way to interpret modal formulas as arithmetical statements. An arithmetical realization of the language of $\mathbf{G L}$ for the theory $T$ is a function $*$ that maps propositional variables to arithmetical sentences. This can be naturally extended to any modal formula $\varphi$. In particular, the modal operator $\square$ is mapped to the Gödel provability predicate $\square_{T}$. Hence, we have the following:

- $(\varphi \rightarrow \psi)^{*}=\varphi^{*} \rightarrow \psi^{*}$;

- $(\neg \varphi)^{*}=\neg \varphi^{*}$;

- $(\square \varphi)^{*}=\square_{T} \varphi^{*}$.

Clearly, we can see that for each modal formula $\varphi$ and any arithmetical realization $*$, if $\mathbf{G L} \vdash \varphi$ then $\mathrm{EA}^{+} \vdash(\varphi)^{*}$. The converse implication holds by the arithmetical completeness theorem for GL due to Solovay [35]:

Theorem 1.4.1. For any elementarily presented theory $T$ which is $\Sigma_{1}^{0}$-sound and contains EA we have that:

GL $\vdash \varphi \Longleftrightarrow T \vdash(\varphi)^{*}$, for every realization $*$ of the variables in $\varphi$.

Apart from arithmetical semantics, GL also has relational semantics which provide a powerful tool to help us in the study of provability logic.

The relational semantics we shall discuss in this dissertation are understood in the usual way. A frame is a pair $\mathcal{F}=\langle W, R\rangle$ consisting of a nonempty set $W$ together with $R$, a binary relation on $W$ known as the $a c$ cessibility relation. The elements of $W$ are called worlds. A frame $\mathcal{F}=\langle W, R\rangle$ is said to be finite iff $W$ is finite. Moreover, sometimes $\mathcal{F}$ is said to have some property of its corresponding accessibility relation e.g. $\mathcal{F}$ is reflexive iff $R \subseteq W^{2}$ is reflexive. A valuation $v$ on a set $W$ is a function $v: W \times \operatorname{Var} \rightarrow$ $\{0,1\}$ where $\operatorname{Var}$ is the set of propositional variables. A valuation assigns a truth value to each propositional variable at each world of $W$. A Kripke 
model is a triple $\mathcal{M}:=\langle W, R, v\rangle$ where $\langle W, R\rangle$ is a frame and $v$ a valuation on $W$. We say that a model is finite iff the frame on which is based is finite. Analogously, we say that a model is reflexive, transitive, etc iff the the frame on which is based is reflexive, transitive, etc.

For each modal formula $\varphi$, each model $\mathcal{M}:=\langle W, R, v\rangle$ and each $x \in W$, we inductively define the relation

$$
\mathcal{M}, x \Vdash \varphi
$$

as follows:

- $\mathcal{M}, x \Vdash \top$;

- $\mathcal{M}, x \Vdash p$ iff $v(x, p)=1$, for $p \in \operatorname{Var}$;

- $\mathcal{M}, x \Vdash \neg \varphi$ iff $\mathcal{M}, x \Vdash \varphi$;

- $\mathcal{M}, x \Vdash \varphi \wedge \psi$ iff $\mathcal{M}, x \Vdash \varphi$ and $\mathcal{M}, x \Vdash \psi$;

- $\mathcal{M}, x \Vdash \square \varphi$ iff for all $y \in W$ such that $x R y$, we have that $\mathcal{M}, y \Vdash \varphi$.

The following theorem establishes the completeness of GL with respect to finite, transitive and irreflexive models.

Theorem 1.4.2. For any formula $\varphi$ :

$$
G L \vdash \varphi \Longleftrightarrow \forall x \in W, \mathcal{M}, x \Vdash \varphi,
$$

for every finite, transitive and irreflexive model $\mathcal{M}:=\langle W, R, v\rangle$.

\section{GLP}

As we saw in Section 1.1, apart from consistency statements and the standard provability predicate, we can define the notions of $n$-consistency with their dual $n$-provability predicate. The logic that fully describes these operators is $\mathbf{G L P}_{\omega}$; a polymodal version of $\mathbf{G L}$ wich contains modalities [ $n$ ] and $\langle n\rangle$ for each $n<\omega$. This system was introduced by Japaridze in [31] and later, extended to a class-sized logic by Beklemishev in [7] where for each $\alpha \in$ Ord we have modalities $\langle\alpha\rangle$ and $[\alpha]$.

Given $\Lambda \in$ Ord, we can consider fragments $\mathbf{G L P}_{\Lambda}$ of GLP by restricting our signature to modalities $[\alpha]$ for $\alpha<\Lambda$. The logic $\mathbf{G L P}_{\Lambda}$ is then given by the following rules and axioms:

1. all propositional tautologies,

2. $[\alpha](\phi \rightarrow \psi) \rightarrow([\alpha] \phi \rightarrow[\alpha] \psi)$ for all $\alpha<\Lambda$, 
3. $[\alpha]([\alpha] \phi \rightarrow \phi) \rightarrow[\alpha] \phi$ for all $\alpha<\Lambda$,

4. $[\beta] \phi \rightarrow[\alpha] \phi$ for $\beta<\alpha<\Lambda$,

5. $\langle\beta\rangle \phi \rightarrow[\alpha]\langle\beta\rangle \phi$ for $\beta<\alpha<\Lambda$,

6. modus ponens and

7. necessitation for each $[\alpha]$.

$\mathbf{G L P}_{\omega}$ is sound and complete with respect to its natural arithmetical realization in terms of $n$-provability. Moreover, one of the main applications of GLP is that its closed fragment can be used to perform a $\Pi_{1}^{0}$-ordinal analysis of PA and related systems [5]. On the other hand, the main disadvantage of GLP or even the smallest polymodal fragment, $\mathbf{G L P}_{2}$, is that it is incomplete for its relational semantics. Without propositional variables, Ignatiev built a relational model in which every consistent formula of variable-free $\mathbf{G L P}_{\omega}$ is satisfied [30], and Fernández-Duque and Joosten extended this to variable-free GLP over the ordinals.

\section{The Reflection Calculus}

GLP gained much interest due to Beklemishev's proof-theoretic applications [5]; however, from a modal logic point of view, it is not an easy system to work with. To this end, in $[17,8,9]$ Beklemishev and Dashkov introduced the system called Reflection Calculus, RC, that axiomatizes the fragment of $\mathbf{G L P}_{\omega}$ consisting of unnested implications of strictly positive formulas. This system is much simpler than $\mathbf{G L P}_{\omega}$ but yet expressive enough to maintain its main proof-theoretic applications.

Similar to $\mathbf{G L P}_{\Lambda}$, the signature of $\mathbf{R C}_{\Lambda}$ contains modalities of the form $\langle\alpha\rangle$ for $\alpha \in \Lambda$. However, since this system only considers strictly positive formulas, the signature does not contain negation, disjunction or modalities $[\alpha]$. Thus, the set of formulas $\mathbb{F}_{\mathbf{R C}_{\Lambda}}$ in this signature is defined by the following grammar:

$$
\varphi:=\top|p|(\varphi \wedge \psi) \mid\langle\alpha\rangle \varphi \text { for } \alpha \in \Lambda .
$$

Next we define a consequence relation over $\mathbb{F}_{\mathbf{R C}_{\Lambda}}$. For the purposes of this presentation, a deductive calculus is a pair $\mathbf{X}=\left(\mathbb{F}_{\mathbf{X}}, \vdash_{\mathbf{X}}\right)$ such that $\mathcal{F}_{\mathbf{X}}$ is some set, the language of $\mathbf{X}$, and $\vdash_{\mathbf{X}} \subseteq \mathbb{F}_{\mathbf{X}} \times \mathbb{F}_{\mathbf{X}}$. We write $\varphi \cong \mathbf{X} \psi$ for $\varphi \vdash_{\mathbf{X}} \psi$ and $\psi \vdash_{\mathbf{X}} \varphi$. We will omit the subscript $\mathbf{X}$ when this does not lead to confusion, including in the definition below, where $\vdash$ denotes $\vdash_{\mathbf{R C}_{\Lambda}}$.

Definition 1.4.3. Given an ordinal $\Lambda$, the calculus $\mathbf{R C}_{\Lambda}$ over $\mathbb{F}_{\mathbf{R}} \mathbf{C}_{\Lambda}$ is given by the following set of axioms and rules:

Axioms: 
1. $\varphi \vdash \varphi, \varphi \vdash \top$;

2. $\varphi \wedge \psi \vdash \varphi, \varphi \wedge \psi \vdash \psi$;

3. $\langle\alpha\rangle\langle\alpha\rangle \varphi \vdash\langle\alpha\rangle \varphi$;
4. $\langle\alpha\rangle \varphi \vdash\langle\beta\rangle \varphi \quad$ for $\alpha>\beta$;

5. $\langle\alpha\rangle \varphi \wedge\langle\beta\rangle \psi \vdash\langle\alpha\rangle(\varphi \wedge\langle\beta\rangle \psi)$

for $\alpha>\beta$.

Rules:

1. If $\varphi \vdash \psi$ and $\varphi \vdash \chi$, then $\varphi \vdash \psi \wedge \chi$;

2. If $\varphi \vdash \psi$ and $\psi \vdash \chi$, then $\varphi \vdash \chi$;

3. If $\varphi \vdash \psi$, then $\langle\alpha\rangle \varphi \vdash\langle\alpha\rangle \psi$;

The reflection calculus has natural arithmetical [25], Kripke [17, 9], algebraic [11] and topological $[13,19,29,30]$ interpretations for which it is sound and complete, but in this dissertation we will work exclusively with reflection calculi from a syntactical perspective. Other variants of the reflection calculus have been proposed, for example working exclusively with worms [18] or allowing additional conservativity operators [15, 10].

This suffices to define the consistency ordering between worms, a crucial ingredient in Beklemishev's proof-theoretic analysis.

\section{Worms and the consistency ordering}

We review the consistency ordering between worms, along with some of their basic properties.

Definition 1.4.4. Fix an ordinal $\Theta<\Lambda$. The set $\mathbb{W}_{\Theta}$ of worms in $\mathbb{F}_{\mathbf{R C}_{\Lambda}}$ is recursively defined as follows:

1. $\top \in \mathbb{W}_{\Theta} ;$

2. If $A \in \mathbb{W}_{\Theta}$ and $\alpha<\Theta$, then $\langle\alpha\rangle A \in \mathbb{W}_{\Theta}$.

Similarly, we inductively define for each $\alpha \in \Theta$ the set of worms $\mathbb{W}_{\Theta}^{\geq \alpha}$ where all ordinals are at least $\alpha$ :

1. $\top \in \mathbb{W}_{\Theta}^{\geq \alpha} ;$

2. If $A \in \mathbb{W}_{\Theta}^{\geq \alpha}$ and $\beta \geq \alpha$, then $\langle\beta\rangle A \in \mathbb{W}_{\Theta}^{\geq \alpha}$.

Definition 1.4.5. Let $A=\left\langle\xi_{1}\right\rangle \ldots\left\langle\xi_{n}\right\rangle \top$ and $B=\left\langle\zeta_{1}\right\rangle \ldots\left\langle\zeta_{m}\right\rangle \top$ be worms. Then, define $A B=\left\langle\xi_{1}\right\rangle \ldots\left\langle\xi_{n}\right\rangle\left\langle\zeta_{1}\right\rangle \ldots\left\langle\zeta_{m}\right\rangle \top$. Given an ordinal $\lambda$, define $\lambda \uparrow A$ to be $\left\langle\lambda+\xi_{1}\right\rangle \ldots\left\langle\lambda+\xi_{n}\right\rangle \top$.

Often we will want to put an extra ordinal between two worms, and we write $B\langle\lambda\rangle A$ for $B(\langle\lambda\rangle A)$. Next, we define the consistency ordering between worms. 
Definition 1.4.6. Given ordinals $\beta, \Theta<\Lambda$, we define a relation $<_{\beta}$ on $\mathbb{W}_{\Theta}^{\geq \beta}$ by $B<_{\beta} A$ if and only if $A \vdash\langle\beta\rangle B$. We also define $B \leq_{\beta} A$ if $B<_{\beta} A$ or $B \cong A$.

The ordering $\leq_{0}$ has some nice properties.

Theorem 1.4.7. For any ordinal $\Theta$, the relation $\leq_{0}$ is a pre-well-order on $\mathbb{W}_{\Theta}$.

Note that $\leq_{0}$ fails to be a linear order merely because it is not antisymmetric. To get around this, one may instead consider worms modulo provable equivalence. Alternately, as Beklemishev has done [7], one can choose a canonical representative for each worm.

Definition 1.4.8 (Beklemishev Normal Form). A worm $A \in \mathbb{W}$ is defined recursively to be in BNF if either

1. $A=\mathrm{\top}$, or

2. $A:=A_{k}\langle\alpha\rangle A_{k-1}\langle\alpha\rangle \ldots\langle\alpha\rangle A_{0}$ with

- $\alpha=\min \mathcal{S}(A)$

- $k \geq 1$;

- $A_{i} \in \mathbb{W}_{\Lambda}^{\geq \alpha+1}$, for $i \leq k$;

such that $A_{i} \in B N F$ and $A_{i+1} \leq_{\alpha+1} A_{i}$ for each $i<k$.

This definition essentially mirrors that of Cantor normal forms for ordinals. The following was proven in [7] and [12].

Theorem 1.4.9. Given any worm $A$ there is a unique $A^{\prime} \in B N F$ such that $A \cong A^{\prime}$.

Thus, with the help of BNF we have the following result:

Theorem 1.4.10. For any ordinal $\Theta$, the relation $\leq_{0}$ is a well-order on $\mathbb{W}_{\Theta} \cap B N F$.

The consistency ordering between worms allows us to compute the ordertype of "finite" worms i.e. $\mathbb{W}_{\omega}$. The elements of $\mathbb{W}_{\omega}$ provide an alternative ordinal notation for ordinals up to $\varepsilon_{0}$. Let us introduce first the following operations on worms: 'promotion' $(\uparrow)$ and 'demotion' $(\downarrow)$.

Definition 1.4.11. Let $\varphi \in \mathbb{F}_{\mathbf{R C}_{\Lambda}}$ and $\lambda$ an ordinal. We define $\lambda \uparrow \varphi$ to be the result of replacing every ordinal $\alpha$ appearing in $\varphi$ by $\lambda+\alpha$. Similarly define $\lambda \downarrow \varphi$ by replacing every occurrence of $\alpha$ by $-\lambda+\alpha$.

Now we can formulate a simple correspondence between $\mathbb{W}_{\omega} \cap \mathrm{BNF}$ and the ordinals below $\varepsilon_{0}$.

Theorem 1.4.12. The map o: $\mathbb{W}_{\omega} \cap B N F \rightarrow \varepsilon_{0}$ is surjective and satisfies

1. $o(\top)=0$, and; 
2. $o((1 \uparrow A)\langle 0\rangle B)=o(B)+\omega^{o(A)}$.

We can go further and consider worms which contain modalities with transfinite ordinals as entries. Fortunately, Theorem 1.4.10 holds for any set of worms, even those which have transfinite entries. Thus, we can extend our previous theorem to all of $\mathbb{W}$ obtaining a complete calculus for $o$.

Theorem 1.4.13. Let $A, B \in \mathbb{W}$ and $\alpha \in$ Ord:

1. $o(\top)=0$, and;

2. $o(A\langle 0\rangle B)=o(B)+1+o(A)$;

3. $o(\alpha \uparrow A)=e^{\alpha}(o(A))$.

Finally, we mention a useful property of $o$ proven in [20] where max $A$ is the greatest ordinal appearing in $A$.

Lemma 1.4.14. Let $A \neq \top$ be a worm and $\mu$ an ordinal. Then,

1. if $\mu \leq \max A$, then $o(\langle\mu\rangle \top) \leq o(A)$, and

2. if $\max A<\mu$, then $o(A)<o(\langle\mu\rangle \top)$. 


\section{The Turing-Schmerl Calculus}

In this Chapter we introduce a propositional modal language using ordinal modalities, which are modalities of the form $\left\langle n^{\alpha}\right\rangle$ where $\alpha \in \Lambda$ for some fixed ordinal $\Lambda$ and $n \in \omega$ (named exponent and base, respectively). Also, we will introduce the system TSC (for Turing-Schmerl Calculus) in this language.

First rank inhabitants of TSC are the so-called monomial normal forms (MNF's for short). We will show that each formula is equivalent to such a MNF.

\subsection{Signature and Ordinal Modalities}

We shall work with the closed fragment of a strictly positive propositional modal signature. From now on, we use $\Lambda$ to denote some fixed ordinal. Moreover, if we shall speak of $\Lambda$ in a formalized setting, we shall tacitly assume that $\Lambda$ is some elementary well-order the properties of which are readily provable in a weak base theory. Further, we require some properties of $\Lambda$ that are collected in the following remark.

Remark 2.1.1. As we shall see in the axioms of our logic, they only make sense if the ordinals occurring in them are available. As such, the usable closure conditions on $\Lambda$ naturally suggest themselves. Since it suffices to require that for $n<\omega$ that $\alpha, \beta<\Lambda \Rightarrow \alpha+e^{n}(\beta)<\Lambda$, we shall for the remainder assume that $\Lambda$ is an $\varepsilon$-number, that is, a fixpoint of e whence $e(\Lambda)=\Lambda=\omega^{\Lambda}$.

The modal signature we are going to work with consists of one constant symbol $T$, one logical connective $\wedge$ and a set of modal connectives $\mathcal{M}:=$ $\left\{\left\langle n^{\alpha}\right\rangle: n<\omega\right.$ and $\left.\alpha<\Lambda\right\}$, named ordinal modalities. We call $n$ the base and $\alpha$ the exponent. The set of formulas in this language is defined as follows: 
Definition 2.1.2. By $\mathbb{F}_{T S C}$ we denote the smallest set such that:

I) $T \in \mathbb{F}_{T S C}$;

II) If $\varphi, \psi \in \mathbb{F}_{T S C}$ then $(\varphi \wedge \psi) \in \mathbb{F}_{T S C}$;

III) If $\varphi \in \mathbb{F}_{\text {TSC }}, n<\omega$ and $\alpha<\Lambda$ then $\left\langle n^{\alpha}\right\rangle \varphi \in \mathbb{F}_{\text {TSC }}$.

In the next chapter we shall see how these formulas can be associated to arithmetical theories in a natural way. To have some control over the nature of these theories, for any formula $\psi$ in the signature we define the following two functions returning the set of base and exponent elements respectively of any modality occurring in $\psi$. That is:

Definition 2.1.3. Given a formula $\psi$, by $N-\bmod (\psi)$ we denote the set of natural numbers corresponding to the base element in each modality that occurs in $\psi$, i.e.,

I) $N-\bmod (\top)=\varnothing$;

II) $N-\bmod (\varphi \wedge \psi)=N-\bmod (\varphi) \cup N-\bmod (\psi)$;

III) $N-\bmod \left(\left\langle n^{\alpha}\right\rangle \psi\right)=\{n\} \cup N-\bmod (\psi)$.

Analogously, by $O-\bmod (\psi)$ we denote the set of ordinals below $\Lambda$ corresponding to the exponent element of each modality in $\psi$, i.e.,

I) $O-\bmod (\top)=\varnothing$;

II) $O-\bmod (\varphi \wedge \psi)=\operatorname{O} \bmod (\varphi) \cup \operatorname{O}-\bmod (\psi)$;

III) $O-\bmod \left(\left\langle n^{\alpha}\right\rangle \psi\right)=\{\alpha\} \cup \operatorname{O} \bmod (\psi)$.

\section{Ordinal worms and restricted sets of formulas}

Within $\mathbb{F}_{\text {TSC }}$ we can find some special formulas, named Ordinal Worms (OWs), which are defined as follows:

Definition 2.1.4 (Ordinal Worms, $\mathbb{W}^{\text {Ord }}$ ). The set of OWs denoted by $\mathbb{W}^{\text {Ord }}$ is inductively defined as:

I) $\top \in \mathbb{W}^{\text {Ord }}$;

II) if $B \in \mathbb{W}^{\text {Ord }}$, then $\left\langle n^{\alpha}\right\rangle B \in \mathbb{W}^{\text {Ord }}$ for any $n<\omega$ and $\alpha<\Lambda$.

In order to control the complexity of the theories corresponding to our formulas, for any $n<\omega$ we define the set of formulas $\mathbb{F}_{<n}$ as follows:

Definition 2.1.5. $\mathbb{F}_{<n}$ is the smallest set such that:

I) $T \in \mathbb{F}_{<n \text { i }}$; 
II) if $\varphi, \psi \in \mathbb{F}_{<n}$ then $(\varphi \wedge \psi) \in \mathbb{F}_{<n}$;

III) if $\varphi \in \mathbb{F}_{<n}$ then $\left\langle m^{\beta}\right\rangle \varphi \in \mathbb{F}_{<n}$ for all $m<n$ and $\beta<\Lambda$.

This last set of formulas will have some special relevance when trying to capture some conservativity results.

From now on, we let Greek letters $\alpha, \beta, \gamma, \ldots$ denote ordinals below $\Lambda$, Greek lower case in the middle of the alphabet like $\varphi, \psi, \chi$ denote formulas, and capital latin letters $A, B, C, \ldots$ denote OWs. Also, given $A \in \mathbb{W}^{\text {Ord }}$ of the form $\left\langle n_{0}^{\alpha_{0}}\right\rangle \ldots\left\langle n_{k}^{\alpha_{k}}\right\rangle T$, and $\varphi \in \mathbb{F}_{\text {TSC }}$ by $A \varphi$ we denote the formula $\psi$ obtained by the concatenation $\left\langle n_{0}^{\alpha_{0}}\right\rangle \ldots\left\langle n_{k}^{\alpha_{k}}\right\rangle \varphi$.

\section{Monomial and increasing normal forms}

In this subsection we consider two different kinds of special formulas named monomial normal forms and increasing normal forms. Formulas in monomial normal form are used in the axiomatization of the calculus TSC as introduced in the next section and play an important role in this thesis.

Later we will show that for every formula $\psi$ in monomial normal form there is a unique equivalent (modulo TSC) OW $A$ in increasing normal form and vice versa. Moreover, we shall show that for any formula $\varphi$ in the above signature, there is a unique equivalent $\psi$ in monomial normal form. These uniqueness claims shall be proved after proving the arithmetical soundness.

Definition 2.1.6. The set Mon of monomials is defined as the set of all OWs obtained by a single iteration of an ordinal modality over $T$. Moreover, we shall focus on those which have an exponent greater that zero i.e., Mon $:=\left\{\left\langle n^{\alpha}\right\rangle \top \in\right.$ $\mathbb{W}^{O n}$ : for some $n<\omega$ and $\left.0<\alpha<\Lambda\right\}$.

Monomial normal forms are conjuntions of monomials with an additional condition on the occuring exponents. To formulate this condition we make use of the hyper-exponential functions introduced in Definition 1.2.10 of Chapter 1, Section 1.2.

Definition 2.1.7. The set of formulas in monomial normal form, MNF, is inductively defined as follows:

I) $T \in M N F$;

II) if $A \in$ Mon then $A \in M N F$;

III) if (a) $\left\langle n_{0}^{\alpha_{0}}\right\rangle \top \wedge \ldots \wedge\left\langle n_{k}^{\alpha_{k}}\right\rangle \top \in M N F$;

(b) $n<n_{0}$;

(c) $\alpha$ is of the form $e^{n_{0}-n}\left(\alpha_{0}\right) \cdot(2+\beta)$ for some $\beta<\Lambda$, 
then $\left\langle n^{\alpha}\right\rangle \top \wedge\left\langle n_{0}^{\alpha_{0}}\right\rangle \top \wedge \ldots \wedge\left\langle n_{k}^{\alpha_{k}}\right\rangle \top \in M N F$.

Thus, monomial normal forms are conjunctions of monomials where the base of the modalities run in increasing order from left to right. The technical Item III) (c) is there to ensure that only monomials are included in the MNF if they add new information to the MNF that was not already implicit by one of the other monomials.

Remark 2.1.8. We can observe that when we chop the left-most monomial of a $M N F$, the resulting formula remains a MNF. Hence if $\left\langle n^{\alpha}\right\rangle \top \wedge \psi \in M N F$, we have that $\psi \in M N F$.

As we shall later see, there is another natural class of formulas that provides us with alternative normal forms. We define this set already here.

Definition 2.1.9. The set of formulas in increasing normal form, INF, is inductively defined as follows:
I) $T \in I N F$;
II) if $A \in$ Mon then $A \in I N F$;
III) if $\left\langle m^{\beta}\right\rangle A \in I N F, n<m$ and $0<\alpha$, then $\left\langle n^{\alpha}\right\rangle\left\langle m^{\beta}\right\rangle A \in I N F$.

\subsection{The Turing-Schmerl Calculus}

In this section we introduce the logic TSC in this modal language whose main goal is to express valid relations that hold between the corresponding Turing progressions.

We will use the following notation: by $\varphi \equiv \psi$ we will denote that both $\varphi \vdash \psi$ and $\psi \vdash \varphi$ are derivable. Analogously, by $\varphi \equiv_{n} \psi$ we denote that for any formula $\chi \in \mathbb{F}_{<n+1}$, we have that $\varphi \vdash \chi$ iff $\psi \vdash \chi$, i.e., $\varphi$ and $\psi$ share the same $\mathbb{F}_{<n+1}$ consequences. Also, by convention we take that for any $n$, the formula $\left\langle n^{0}\right\rangle \varphi$ is just $\varphi$.

Definition 2.2.1. TSC is given by the following set of axioms and rules:

Axioms:

1. $\varphi \vdash \varphi, \varphi \vdash \top$;

2. $\varphi \wedge \psi \vdash \varphi, \varphi \wedge \psi \vdash \psi$;

3. Monotonicity axioms: $\left\langle n^{\alpha}\right\rangle \varphi \vdash\left\langle n^{\beta}\right\rangle \varphi$, for $\beta \leq \alpha$;

4. Co-additivity axioms: $\left\langle n^{\alpha+\beta}\right\rangle \varphi \equiv\left\langle n^{\beta}\right\rangle\left\langle n^{\alpha}\right\rangle \varphi$; 
5. Reduction axioms: $\left\langle(m+n)^{\alpha}\right\rangle \varphi \vdash\left\langle m^{e^{n}(\alpha)}\right\rangle \varphi$;

6. Schmerl axioms:

$$
\left\langle n^{\alpha}\right\rangle\left(\left\langle n_{0}^{\alpha_{0}}\right\rangle \top \wedge \psi\right) \equiv\left\langle n^{e^{n_{0}-n}\left(\alpha_{0}\right) \cdot(1+\alpha)}\right\rangle \top \wedge\left\langle n_{0}^{\alpha_{0}}\right\rangle \top \wedge \psi
$$

for $n<n_{0}$ and $\left\langle n_{0}^{\alpha_{0}}\right\rangle \top \wedge \psi \in M N F$.

Rules:

1. If $\varphi \vdash \psi$ and $\varphi \vdash \chi$, then $\varphi \vdash \psi \wedge \chi$;

2. If $\varphi \vdash \psi$ and $\psi \vdash \chi$, then $\varphi \vdash \chi$;

3. If $\varphi \vdash \psi$, then $\left\langle n^{\alpha}\right\rangle \varphi \vdash\left\langle n^{\alpha}\right\rangle \psi$;

4. If $\varphi \vdash \psi$, then $\left\langle n^{\alpha}\right\rangle \varphi \wedge\left\langle m^{\beta+1}\right\rangle \psi \vdash\left\langle n^{\alpha}\right\rangle\left(\varphi \wedge\left\langle m^{\beta+1}\right\rangle \psi\right)$ for $m<n$.

It is worth mentioning the special character of Axioms 5 and 6 since both axioms are modal formulations of principles related to Schmerl's finestructure theorem, also known as Schmerl's formulas (see [34] and [4]), that we already mentioned in the introduction. These results are formulated precisely in Proposition 3.2.5 of this thesis.

The following proposition expresses some useful properties that can be easily proved.

Proposition 2.2.2. For any $A \in \mathbb{W}^{\text {Ord }}$ and $\varphi, \psi \in \mathbb{F}_{T S C}$ :

1. If $\varphi \vdash \psi$, then $A \varphi \vdash A \psi$;

2. $A \varphi \vdash \varphi \wedge A$;

3. $\left\langle(m+n)^{\alpha}\right\rangle \varphi \vdash\left\langle m^{\alpha}\right\rangle \varphi$;

4. $\left\langle n^{\alpha}\right\rangle(\varphi \wedge \psi) \wedge\left\langle m^{\beta+1}\right\rangle \varphi \equiv\left\langle n^{\alpha}\right\rangle\left(\left\langle m^{\beta+1}\right\rangle \varphi \wedge \psi\right)$ for $m<n$;

5. $\left\langle n^{\alpha}\right\rangle \varphi \wedge \psi \equiv\left\langle n^{\alpha}\right\rangle(\varphi \wedge \psi)$ for $\psi \in \mathbb{F}_{<n}$ and $O-\bmod (\psi) \subset$ Succ.

Proof. The only non-trivial item is Item 5 which follows from a straightforward induction on the length of $\psi$ using Rule 4 .

\subsection{Normal Forms}

In this section we shall prove that any formula of $\mathbb{F}_{\text {TSC }}$ is equivalent to a formula in monomial normal for -MNF- and also equivalent to a formula in increasing normal form, INF. 


\section{Monomial normal forms versus increasing normal forms}

In this subsection we prove that monomial normal forms and increasing normal forms can easily be transformed into each other. Let us first observe that for each MNF there is an equivalent INF that holds some similarities with the original MNF. From now on we shall make use of the Euclidean algorithm for ordinals. More precisely, for $\beta, \delta \in \operatorname{Lim}$, by $\frac{\beta}{\delta}$ we denote the unique $\alpha$ such that $\delta \cdot \alpha=\beta$, if this exists.

Theorem 2.3.1. For every $\psi \in M N F$ with $\psi:=\left\langle n_{0}^{\alpha_{0}}\right\rangle \top \wedge \ldots \wedge\left\langle n_{k}^{\alpha_{k}}\right\rangle \top$, there is an ordinal worm $A \in I N F$ such that:

1. $\psi \equiv A$;

2. $A:=\left\langle n_{0}^{\beta_{0}}\right\rangle \ldots\left\langle n_{k}^{\beta_{k}}\right\rangle \top$ where:

a) $\beta_{k}=\alpha_{k}$ and,

b) for all $i, 0 \leq i<k$ we have

$$
\beta_{i}=-1+\frac{\alpha_{i}}{e^{n_{i+1}-n_{i}}\left(\alpha_{i+1}\right)} .
$$

Proof. By induction on $k$. The base case is trivial and the inductive case follows from the I.H. and the Schmerl axiom (Axiom 6). Note that by an easy induction we see that the division is well-defined in virtue of Item (III) of Definition 2.1.7.

In particular, this theorem tells us that if we start out with a MNF $\psi$, there is an equivalent INF ordinal worm $A$ where the modality bases are the same. The next theorem tells us that this feature of conserving the modality bases also holds if we go from an ordinal worm $A$ in INF to an equivalent formula $\psi$ in MNF.

Theorem 2.3.2. Let $A:=\left\langle n_{0}^{\beta_{0}}\right\rangle \ldots\left\langle n_{k}^{\beta_{k}}\right\rangle \top \in I N F$. There is $\psi \in M N F$ such that:

1. $A \equiv \psi ;$

2. $\psi:=\left\langle n_{0}^{\alpha_{0}}\right\rangle \top \wedge \ldots \wedge\left\langle n_{k}^{\alpha_{k}}\right\rangle \top$ where

a) $\alpha_{k}=\beta_{k}$ and,

b) for all $i, 0 \leq i<k, \alpha_{i}=e^{n_{i+1}-n_{i}}\left(\beta_{i+1}\right) \cdot\left(1+\beta_{i}\right)$.

Proof. By induction on $k$, with the help of the Schmerl axiom (Axiom 6) for the inductive case. 
The proofs of these theorems actually provide operations $\mathcal{I}$ and $\mathcal{M}$ to go from a formula $\psi$ in MNF to a corresponding $\mathcal{I}(\psi)$ in INF and from an ordinal worm $A$ in INF to a corresponding formula $\mathcal{M}(A)$ in MNF.

Corollary 2.3.3. Given $\psi:=\left\langle n_{0}^{\alpha_{0}}\right\rangle \top \wedge \ldots \wedge\left\langle n_{k}^{\alpha_{k}}\right\rangle \top \in M N F$ and $A$ of the form $\left\langle n_{0}^{\beta_{0}}\right\rangle \ldots\left\langle n_{k}^{\beta_{k}}\right\rangle \top \in I N F$, we have that:

- $\mathcal{M} \circ \mathcal{I}(\psi)=\psi$

- $\mathcal{I} \circ \mathcal{M}(A)=A$;

- If $\mathcal{I}(\psi)=A$, then $\mathcal{I}\left(\left\langle n_{1}^{\alpha_{1}}\right\rangle \top \wedge \ldots \wedge\left\langle n_{k}^{\alpha_{k}}\right\rangle \top\right)=\left\langle n_{1}^{\beta_{1}}\right\rangle \ldots\left\langle n_{k}^{\beta_{k}}\right\rangle \top$.

\section{Monomial normal forms are closed under conjunctions}

In this subsection we will prove that MNF's are closed under taking conjunctions. A first step in doing so is established by the following lemma.

Lemma 2.3.4. Let $\psi:=\left\langle n_{0}^{\alpha_{0}}\right\rangle \top \wedge \ldots \wedge\left\langle n_{k}^{\alpha_{k}}\right\rangle \top \in M N F$ and $n<n_{0}$. For any $\beta<\Lambda$, there is $\psi^{\prime} \in M N F$ such that:

$$
\left\langle n^{\beta}\right\rangle \top \wedge \psi \equiv \psi^{\prime},
$$

with $N-\bmod \left(\psi^{\prime}\right) \subseteq N-\bmod \left(\left\langle n^{\beta}\right\rangle \top \wedge \psi\right)$.

Proof. If $\beta \leq e^{n_{0}-n}\left(\alpha_{0}\right)$, clearly we can take $\psi^{\prime}=\psi$. Otherwise, let $\beta_{\mathrm{CNF}}$ be the Cantor normal form of $\beta$ with base $\omega$ and we write $\beta_{\mathrm{CNF}}:=\beta_{0}+\ldots+\beta_{i}$ with each $\beta_{j}$ non-zero and additively indecomposable and $\beta_{0} \geq \ldots \geq \beta_{i}$.

If for all $j$ with $0 \leq j \leq i$ we have that $\beta_{j} \geq e^{n_{0}-n}\left(\alpha_{0}\right)$ (or equivalently, if $\left.\beta_{i} \geq e^{n_{0}-n}\left(\alpha_{0}\right)\right)$, then it easy to see that $\left\langle n^{\beta}\right\rangle \top \wedge \psi \in$ MNF. If on the contrary, there is $j$ with $0<j \leq i$ such that $\beta_{j}<e^{n_{0}-n}\left(\alpha_{0}\right)$ and $\beta_{j-1} \geq e^{n_{0}-n}\left(\alpha_{0}\right)$, then we reason as follows:

$$
\begin{aligned}
\left\langle n^{\beta}\right\rangle \top \wedge \psi & \equiv\left\langle n^{\beta}\right\rangle \top \wedge\left\langle n_{0}^{\alpha_{0}}\right\rangle \top \wedge \psi ; \\
& \equiv\left\langle n^{\beta_{0}+\ldots+\beta_{i}}\right\rangle \top \wedge\left\langle n_{0}^{\alpha_{0}}\right\rangle \top \wedge \psi ; \\
& \vdash\left\langle n^{\beta_{0}+\ldots+\beta_{j-1}+1}\right\rangle \top \wedge\left\langle n_{0}^{\alpha_{0}}\right\rangle \top \wedge \psi ; \\
& \vdash\left\langle n_{0}^{\alpha_{0}}\right\rangle\left\langle n^{\beta_{0}+\ldots+\beta_{j-1}+1}\right\rangle \top \wedge \psi ; \\
& \vdash\left\langle n^{e^{n_{0}-n}\left(\alpha_{0}\right)}\right\rangle\left\langle n^{\beta_{0}+\ldots+\beta_{j-1}+1}\right\rangle \top \wedge \psi ; \\
& \vdash\left\langle n^{\beta_{0}+\ldots+\beta_{j-1}+e^{n_{0}-n}\left(\alpha_{0}\right)}\right\rangle \top \wedge \psi .
\end{aligned}
$$

However, by the monotonicity axiom (Axiom 3) we see that

$$
\left\langle n^{\beta_{0}+\ldots+\beta_{j-1}+e^{n_{0}-n}\left(\alpha_{0}\right)}\right\rangle \top \vdash\left\langle n^{\beta}\right\rangle \top
$$


so that actually $\left\langle n^{\beta_{0}+\ldots+\beta_{j-1}+e^{n_{0}-n}\left(\alpha_{0}\right)}\right\rangle \top \wedge \psi \equiv\left\langle n^{\beta}\right\rangle \top \wedge \psi$. Clearly we have

$$
\left\langle n^{\beta_{0}+\ldots+\beta_{j-1}+e^{n_{0}-n}\left(\alpha_{0}\right)}\right\rangle \top \wedge \psi \in \mathrm{MNF}
$$

and we are done.

We can observe that, in any case, the modality bases occurring in $\psi^{\prime}$ are contained in $\mathrm{N}-\bmod \left(\left\langle n^{\beta}\right\rangle \top \wedge \psi\right)$, and so, $\mathrm{N}-\bmod \left(\psi^{\prime}\right) \subseteq \mathrm{N}-\bmod \left(\left\langle n^{\beta}\right\rangle \top \wedge\right.$ $\psi)$.

We get the following simple corollaries.

Corollary 2.3.5. Any conjunction of monomials $\chi$ is equivalent to some $\psi \in M N F$ with $N-\bmod (\psi) \subseteq N-\bmod (\chi)$.

Proof. By the length of such a conjunction of monomials written with the modality bases from small to large, applying Lemma 2.3.4 at the inductive step.

We can recast this corollary as the first inductive step that each formula is equivalent to a MNF.

Corollary 2.3.6. The monomials normal forms $-M N F^{\prime}$ - are closed under conjunctions.

In the light of this corollary, it only remains to show that MNF's are closed under putting an ordinal-diamond up front of them.

\section{Monomial normal forms are closed under ordinal diamonds}

We shall now see that the collection of monomial normal forms is, modulo provable equivalence, closed under putting ordinal diamonds up front of them. Let us recall Schmerl's axiom scheme:

$$
\left\langle n^{\alpha}\right\rangle\left(\left\langle n_{0}^{\alpha_{0}}\right\rangle \top \wedge \psi\right) \equiv\left\langle n^{e^{n_{0}-n}\left(\alpha_{0}\right) \cdot(1+\alpha)}\right\rangle \top \wedge\left\langle n_{0}^{\alpha_{0}}\right\rangle \top \wedge \psi,
$$

for $n<n_{0}$ and $\left\langle n_{0}^{\alpha_{0}}\right\rangle \top \wedge \psi \in$ MNF. We observe that this axiom tells us that putting a relatively small $\left(n<n_{0}\right)$ non-trivial $(\alpha \geq 1)$ ordinal modality up front a MNF $\left\langle n_{0}^{\alpha_{0}}\right\rangle \top \wedge \psi$ yields a new MNF. To see that putting any ordinal modality up front a MNF is equivalent to some other MNF, we need to study two cases: $n=n_{0}$ and $n>n_{0}$. Let us start with the latter.

Proposition 2.3.7. Let $n, n_{0}$ be natural numbers with $n>n_{0}$. Furthermore, let $\left\langle n_{0}^{\alpha_{0}}\right\rangle \top \wedge\left\langle n_{1}^{\alpha_{1}}\right\rangle \top \wedge \ldots \wedge\left\langle n_{k}^{\alpha_{k}}\right\rangle \top$ with $k \geq 0$ be a conjunction of monomials not necessarily in MNF. The following principle (we use PS for Pseudo Schmerl) is derivable in TSC:

$$
\begin{aligned}
\left\langle n^{\alpha}\right\rangle\left(\left\langle n_{0}^{\alpha_{0}}\right\rangle \top \wedge\right. & \left.\left.\left\langle n_{1}^{\alpha_{1}}\right\rangle \top \wedge \ldots \wedge\left\langle n_{k}^{\alpha_{k}}\right\rangle \top\right) \equiv \quad \text { (PSI }\right) \\
& \left\langle n_{0}^{\alpha_{0}+e^{n-n_{0}}(\alpha)}\right\rangle \top \wedge\left\langle n^{\alpha}\right\rangle\left(\left\langle n_{1}^{\alpha_{1}}\right\rangle \top \wedge \ldots \wedge\left\langle n_{k}^{\alpha_{k}}\right\rangle \top\right) .
\end{aligned}
$$


Note that for the case when $k=0$, the formula $\left\langle n_{1}^{\alpha_{1}}\right\rangle \top \wedge \ldots \wedge\left\langle n_{k}^{\alpha_{k}}\right\rangle \top$ is to be read as the empty conjunction which is $\top$ so that the principle becomes

$$
\left\langle n^{\alpha}\right\rangle\left\langle n_{0}^{\alpha_{0}}\right\rangle \top \equiv\left\langle n_{0}^{\alpha_{0}+e^{n-n_{0}}(\alpha)}\right\rangle \top \wedge\left\langle n^{\alpha}\right\rangle \top .
$$

Proof. For the right-to-left sequent, we apply the following reasoning:

$$
\begin{aligned}
\left\langle n_{0}^{\alpha_{0}+e^{n-n_{0}}(\alpha)}\right\rangle \top \wedge\left\langle n^{\alpha}\right\rangle & \left(\left\langle n_{1}^{\alpha_{1}}\right\rangle \top \wedge \ldots \wedge\left\langle n_{k}^{\alpha_{k}}\right\rangle \top\right) \\
& \vdash\left\langle n_{0}^{\alpha_{0}+1}\right\rangle \top \wedge\left\langle n^{\alpha}\right\rangle\left(\left\langle n_{1}^{\alpha_{1}}\right\rangle \top \wedge \ldots \wedge\left\langle n_{k}^{\alpha_{k}}\right\rangle \top\right) ; \\
& \vdash\left\langle n^{\alpha}\right\rangle\left(\left\langle n_{0}^{\alpha_{0}+1}\right\rangle \top \wedge\left\langle n_{1}^{\alpha_{1}}\right\rangle \top \wedge \ldots \wedge\left\langle n_{k}^{\alpha_{k}}\right\rangle \top\right) ; \\
& \vdash\left\langle n^{\alpha}\right\rangle\left(\left\langle n_{0}^{\alpha_{0}}\right\rangle \top \wedge\left\langle n_{1}^{\alpha_{1}}\right\rangle \top \wedge \ldots \wedge\left\langle n_{k}^{\alpha_{k}}\right\rangle \top\right) .
\end{aligned}
$$

For the left-to-right sequent we can reason as follows:

$$
\begin{aligned}
\left\langle n^{\alpha}\right\rangle\left(\left\langle n_{0}^{\alpha_{0}}\right\rangle \top \wedge\right. & \left.\ldots \wedge\left\langle n_{k}^{\alpha_{k}}\right\rangle \top\right) \\
& \vdash\left\langle n^{\alpha}\right\rangle\left\langle n_{0}^{\alpha_{0}}\right\rangle \top \wedge\left\langle n^{\alpha}\right\rangle\left(\left\langle n_{1}^{\alpha_{1}}\right\rangle \top \wedge \ldots \wedge\left\langle n_{k}^{\alpha_{k}}\right\rangle \top\right) ; \\
& \vdash\left\langle n_{0}^{e^{n-n_{0}}(\alpha)}\right\rangle\left\langle n_{0}^{\alpha_{0}}\right\rangle \top \wedge\left\langle n^{\alpha}\right\rangle\left(\left\langle n_{1}^{\alpha_{1}}\right\rangle \top \wedge \ldots \wedge\left\langle n_{k}^{\alpha_{k}}\right\rangle \top\right) ; \\
& \vdash\left\langle n_{0}^{\alpha_{0}+e^{n-n_{0}}(\alpha)}\right\rangle \top \wedge\left\langle n^{\alpha}\right\rangle\left(\left\langle n_{1}^{\alpha_{1}}\right\rangle \top \wedge \ldots \wedge\left\langle n_{k}^{\alpha_{k}}\right\rangle \top\right) .
\end{aligned}
$$

We now turn to the remaining case when $n=n_{0}$, that is, when we take a Turing progression based on $n_{0}$ consistency of a theory that is axiomatized by a MNF whose smallest modality has base $n_{0}$.

Proposition 2.3.8. Let $\left\langle n_{0}^{\alpha_{0}}\right\rangle \top \wedge \ldots \wedge\left\langle n_{k}^{\alpha_{k}}\right\rangle \top \in M N F$ for $k \geq 1$. The following principle (that we shall call Pseudo Schmerl 2) is derivable in TSC:

$$
\begin{aligned}
\left\langle n_{0}^{\alpha}\right\rangle\left(\left\langle n_{0}^{\alpha_{0}}\right\rangle \top\right. & \left.\wedge \ldots \wedge\left\langle n_{k}^{\alpha_{k}}\right\rangle \top\right) \\
& \equiv\left\langle n_{0}^{\alpha_{0}+e^{n_{1}-n_{0}}\left(\alpha_{1}\right) \cdot \alpha}\right\rangle \top \wedge\left\langle n_{0}^{\alpha}\right\rangle\left(\left\langle n_{1}^{\alpha_{1}}\right\rangle \top \wedge \ldots \wedge\left\langle n_{k}^{\alpha_{k}}\right\rangle \top\right) ; \\
& \equiv\left\langle n_{0}^{\alpha_{0}+e^{n_{1}-n_{0}}\left(\alpha_{1}\right) \cdot \alpha}\right\rangle \top \wedge\left\langle n_{1}^{\alpha_{1}}\right\rangle \top \wedge \ldots \wedge\left\langle n_{k}^{\alpha_{k}}\right\rangle \top .
\end{aligned}
$$

Proof. Note that by means of Theorem 2.3.1 together with the co-additivity axiom (Axiom 4) we have that

$$
\left\langle n_{0}^{\alpha}\right\rangle\left(\left\langle n_{0}^{\alpha_{0}}\right\rangle \top \wedge \ldots \wedge\left\langle n_{k}^{\alpha_{k}}\right\rangle \top\right) \equiv\left\langle n_{0}^{\delta_{0}+\alpha}\right\rangle\left\langle n_{1}^{\delta_{1}}\right\rangle \ldots\left\langle n_{k}^{\alpha_{k}}\right\rangle \top
$$

with $\delta_{i}=-1+\frac{\alpha_{i}}{e^{n_{i+1} n_{i}}\left(\alpha_{i+1}\right)}$ for $0 \leq i<k$. Thus, by Corollary 2.3.3 we get that:

$$
\left\langle n_{0}^{\delta_{0}+\alpha}\right\rangle\left\langle n_{1}^{\delta_{1}}\right\rangle \ldots\left\langle n_{k}^{\alpha_{k}}\right\rangle \top \equiv\left\langle n_{0}^{\delta_{0}+\alpha}\right\rangle\left(\left\langle n_{1}^{\alpha_{1}}\right\rangle \top \wedge \ldots \wedge\left\langle n_{k}^{\alpha_{k}}\right\rangle \top\right) .
$$


Now, we apply the Schmerl axiom (Axiom 6) observing that

$$
e^{n_{1}-n_{0}}\left(\alpha_{1}\right) \cdot\left(1+\delta_{0}+\alpha\right)=e^{n_{1}-n_{0}}\left(\alpha_{1}\right) \cdot\left(1+\left(-1+\frac{\alpha_{0}}{e^{n_{1}-n_{0}}\left(\alpha_{1}\right)}\right)+\alpha\right)
$$

that is,

$e^{n_{1}-n_{0}}\left(\alpha_{1}\right) \cdot\left(1+\delta_{0}+\alpha\right)=e^{n_{1}-n_{0}}\left(\alpha_{1}\right) \cdot\left(\frac{\alpha_{0}}{e^{n_{1}-n_{0}}\left(\alpha_{1}\right)}+\alpha\right)=\alpha_{0}+e^{n_{1}-n_{0}}\left(\alpha_{1}\right) \cdot \alpha$,

to conclude that

$$
\left\langle n_{0}^{\delta_{0}+\alpha}\right\rangle\left\langle n_{1}^{\delta_{1}}\right\rangle \ldots\left\langle n_{k}^{\alpha_{k}}\right\rangle \top \equiv\left\langle n_{0}^{\alpha_{0}+e^{n_{1}-n_{0}}\left(\alpha_{1}\right) \cdot \alpha}\right\rangle \top \wedge\left\langle n_{1}^{\alpha_{1}}\right\rangle \top \wedge \ldots \wedge\left\langle n_{k}^{\alpha_{k}}\right\rangle \top .
$$

Thus,

$$
\begin{aligned}
\left\langle n_{0}^{\alpha}\right\rangle\left(\left\langle n_{0}^{\alpha_{0}}\right\rangle \top \wedge \ldots \wedge\right. & \left.\left\langle n_{k}^{\alpha_{k}}\right\rangle \top\right) \equiv \\
& \left\langle n_{0}^{\alpha_{0}+e^{n_{1}-n_{0}}\left(\alpha_{1}\right) \cdot \alpha}\right\rangle \top \wedge\left\langle n_{1}^{\alpha_{1}}\right\rangle \top \wedge \ldots \wedge\left\langle n_{k}^{\alpha_{k}}\right\rangle \top .
\end{aligned}
$$

Since we have that $\left\langle n_{0}^{\alpha}\right\rangle\left(\left\langle n_{0}^{\alpha_{0}}\right\rangle \top \wedge \ldots \wedge\left\langle n_{k}^{\alpha_{k}}\right\rangle \top\right) \vdash\left\langle n_{0}^{\alpha}\right\rangle\left(\left\langle n_{1}^{\alpha_{1}}\right\rangle \top \wedge \ldots \wedge\right.$ $\left.\left\langle n_{k}^{\alpha_{k}}\right\rangle \top\right)$ and $\left\langle n_{0}^{\alpha}\right\rangle\left(\left\langle n_{1}^{\alpha_{1}}\right\rangle \top \wedge \ldots \wedge\left\langle n_{k}^{\alpha_{k}}\right\rangle \top\right) \vdash\left\langle n_{1}^{\alpha_{1}}\right\rangle \top \wedge \ldots \wedge\left\langle n_{k}^{\alpha_{k}}\right\rangle \top$, we also get that

$$
\begin{aligned}
\left\langle n_{0}^{\alpha}\right\rangle\left(\left\langle n_{0}^{\alpha_{0}}\right\rangle \top\right. & \left.\wedge \ldots \wedge\left\langle n_{k}^{\alpha_{k}}\right\rangle \top\right) \\
& \vdash\left\langle n_{0}^{\alpha_{0}+e^{n_{1}-n_{0}}\left(\alpha_{1}\right) \cdot \alpha}\right\rangle \top \wedge\left\langle n_{0}^{\alpha}\right\rangle\left(\left\langle n_{1}^{\alpha_{1}}\right\rangle \top \wedge \ldots \wedge\left\langle n_{k}^{\alpha_{k}}\right\rangle \top\right) ; \\
& \vdash\left\langle n_{0}^{\alpha_{0}+e^{n_{1}-n_{0}}\left(\alpha_{1}\right) \cdot \alpha}\right\rangle \top \wedge\left\langle n_{1}^{\alpha_{1}}\right\rangle \top \wedge \ldots \wedge\left\langle n_{k}^{\alpha_{k}}\right\rangle \top,
\end{aligned}
$$

which concludes the proof.

This proposition allows us to incorporate a small modality into a MNF that starts with the same modality. We note that the degenerate version of the proposition would be with $k=0$ which is covered by the co-additivity axiom (Axiom 4): $\left\langle n_{0}^{\alpha}\right\rangle\left\langle n_{0}^{\alpha_{0}}\right\rangle \top \equiv\left\langle n_{0}^{\alpha_{0}+\alpha}\right\rangle \top$.

Theorem 2.3.9. The set of MNF's is, modulo provable equivalence, closed under putting ordinal modalities up front.

Proof. For an arbitrary MNF $\left\langle n_{0}^{\alpha_{0}}\right\rangle \top \wedge \ldots \wedge\left\langle n_{k}^{\alpha_{k}}\right\rangle \top$ and arbitrary ordinal modality $\left\langle n^{\alpha}\right\rangle$ we have to see that $\left\langle n^{\alpha}\right\rangle\left(\left\langle n_{0}^{\alpha_{0}}\right\rangle \top \wedge \ldots \wedge\left\langle n_{k}^{\alpha_{k}}\right\rangle \top\right)$ is equivalent to some MNF. The proof follows directly from our earlier results.

By Pseudo Schmerl 1, Proposition 2.3.7, we can 'pull' the ordinal modality $\left\langle n^{\alpha}\right\rangle$ over the smaller ordinal modalities of $\left\langle n_{0}^{\alpha_{0}}\right\rangle \top \wedge \ldots \wedge\left\langle n_{k}^{\alpha_{k}}\right\rangle \top$ until $n \leq n_{i}$ for some $0 \leq i \leq k$.

In case $n=n_{i}$ we can apply (PS2) (or the co-additivity axiom (Axiom 4) for the degenerate case) and we can apply directly Schmerl's axiom when $n<n_{i}$. The result yields us a conjunction of monomials which by Corollary 2.3.5 we know is equivalent to some MNF. 


\section{Each formula is equivalent to a normal form}

In this subsection, we shall combine all the earlier results of this section to obtain the, by now, simple corollary that every formula $\varphi$ is equivalent to a MNF formula. As a corollary we will obtain that $\varphi$ is also equivalent to an ordinal worm $A$ in INF. However, to establish the uniqueness of MNF's and INF's we need non-derivability results. And, without modal semantics, the arithmetical soundness of the system is required. Hence, the uniqueness will be discussed later.

Theorem 2.3.10. For every formula $\varphi$, there is $\psi \in M N F$ such that $\varphi \equiv \psi$.

Proof. By induction on $\varphi$ applying Corollary 2.3.6 and Theorem 2.3.9 for the conjunctive and ordinal modality cases, respectively.

Consequently, we see that each formula also has an INF.

Corollary 2.3.11. For every formula $\varphi$, there is $A \in I N F$ such that $\varphi \equiv A$.

Proof. By combining Theorems 2.3.1 and 2.3.10. 



\section{CHAPTER}

\section{Arithmetical Semantics}

We dedicate this chapter to introduce the arithmetical semantics of TSC. First we shall make the link between our logic and graded Turing progressions more precise by introducing the Formalized Turing Progression (FTP) Interpretation that defines an arithmetical translation between modal formulas and arithmetical formulas. This allows us to fix an interpretation for the modal sequents within $\mathrm{EA}^{+}$.

In Section 3.2 we prove the soundness of TSC with respect to this interpretation. In Section 3.3, we prove some conservativity results and a characterization for the derivability between MNF. With these results we prove the arithmetical completeness of the system.

The content of this chapter can be found in [27].

\subsection{Arithmetical interpretation of modal formulas}

As we already mentioned in the introduction, we want our modal formulas to directly denote arithmetical theories rather than individual arithmetical sentences. Therefore, we interpret our modal formulae in terms of the $\tau$-formulae presented in Section 1.1 of Chapter 1. Moreover, the conjunction of modal formulas is intended to mean the union of theories. Hence, we map the interpretation of modal conjunctions to the disjunction of the respective interpretations.

Definition 3.1.1. An arithmetical interpretation for $\mathbb{F}_{T S C}$ is a map $*: \mathbb{F}_{T S C} \longrightarrow$ Form $_{\mathcal{L}_{\mathbb{N}}}$ inductively defined as follows:

1. $(\top)^{*}(x)=\epsilon(x)$; 
2. $(\varphi \wedge \psi)^{*}(x)=(\varphi)^{*}(x) \vee(\psi)^{*}(x)$;

3. $\left(\left\langle n^{\alpha}\right\rangle \varphi\right)^{*}(x)=\tau_{n}^{\varphi^{*}}(\alpha, x)$.

Note that since $\mathbb{F}_{\text {TSC }}$ has no propositional variables, we can identify a modal formula with its arithmetical interpretation unambiguously and so we will do in the remainder of this dissertation.

Definition 3.1.2. Given a numeration $\sigma$, by $T h_{\sigma}$ we denote the theory of $\sigma$. We say that $\chi \in T h_{\sigma}$ iff $\mathbb{N} \models \sigma(\ulcorner\chi\urcorner)$.

For the sake of clarity, and since we are working in the closed fragment, we will use the following notation: given $\varphi \in \mathbb{F}_{\text {TSC }}$ by $\operatorname{Th}_{\varphi}$ we denote $\operatorname{Th}_{\sigma}$ where $\varphi^{*}(x)=\sigma(x)$, following Definition 3.1.1. If $\varphi^{*}(x)=\epsilon(x)$ we use just $\mathrm{EA}^{+}$instead of $\mathrm{Th}_{\epsilon}$. Also, if $\varphi:=\left\langle n^{\alpha}\right\rangle \psi$ we write $\left(\mathrm{Th}_{\psi}\right)_{n}^{\alpha}$ for the theory of $\left(\left\langle n^{\alpha}\right\rangle \psi\right)^{*}$.

The Formalized Turing Progression Interpretation (FTP) is the representation of provable TSC sequents as the entailment between the corresponding first order theories within $\mathrm{EA}^{+}$via the $\Pi_{2}^{0}$-sentence expressing such derivability. Thus, given $\varphi, \psi \in \mathbb{F}_{\text {TSC }}$ the intended interpretation of $\varphi \vdash \psi$ is the arithmetical statement:

$$
\mathrm{EA}^{+} \vdash \forall x\left(\square \mathrm{Th}_{\psi}(x) \rightarrow \square_{\mathrm{Th}_{\varphi}}(x)\right) .
$$

Hereinafter, we will adopt the following notation: by $T \subseteq U$ we denote the arithmetical formula $\forall x\left(\square_{T}(x) \rightarrow \square_{U}(x)\right)$. Likewise, by $T \equiv U$, we denote the formula $\forall x\left(\square_{T}(x) \leftrightarrow \square_{U}(x)\right)$ and $T \equiv_{n} U$ stands for the formula $\forall x \in \Pi_{n+1}^{0}\left(\square_{T}(x) \leftrightarrow \square_{U}(x)\right)$.

\subsection{Arithmetical Soundness of TSC}

In this section we shall prove that the logic TSC adequately describes graded Turing progressions.

Before proving soundness of TSC under the FTP interpretation we will present some useful facts and tools in this subsection. In Lemma 2.2 of [2] a very simple yet very useful fact is shown: if a formula is provable in some Turing progression, then it is provable in the base theory together with a single consistency statement.

Lemma 3.2.1. Provably in $\mathrm{EA}^{+}$we have that

$$
\forall \alpha \succ 0 \forall x\left(\square_{T_{n}^{\alpha}}(x) \rightarrow \exists \gamma \prec \alpha \square_{T}\left(\operatorname{Con}_{n}\left((T)_{n}^{\dot{\gamma}}\right) \rightarrow x\right)\right) .
$$

The proof of this lemma is elementary as holds for the following. 
Lemma 3.2.2. Let $T$ and $U$ be theories extending EA. For any $n, m<\omega$ and $\delta \preceq \alpha \prec \Lambda$, provably in $\mathrm{EA}^{+}$:

1. $T \subseteq U \rightarrow\left(\operatorname{Con}_{n}(U) \rightarrow \operatorname{Con}_{n}(T)\right) ;$

2. $U \equiv_{n} T \rightarrow\left(\operatorname{Con}_{n}(T) \leftrightarrow \operatorname{Con}_{n}(U)\right)$;

3. $\beta \preceq \gamma \rightarrow(T)_{n}^{\beta} \subseteq(T)_{n}^{\gamma}$;

4. $\operatorname{Con}_{n}\left((T)_{m}^{\alpha}\right) \rightarrow \operatorname{Con}_{n}\left((T)_{m}^{\delta}\right)$.

Proof. All items allow for elementary proofs. For example, reasoning informally in $\mathrm{EA}^{+}$, for Item 1 , assume $T \subseteq U$ and $\operatorname{Con}_{n}(U)$. Now, let $\pi \in \Pi_{n+1}^{0}$ such that $\square_{T} \pi$. By assumption, $\square_{U} \pi$ and by $\operatorname{Con}_{n}(U)$ we get that $\operatorname{Tr}_{n+1}(\pi)$ as was to be shown. Item 2 goes analogously. Item 3 follows directly from Lemma 3.2.1 and Item 4 follows from Item 1 together with the fact that $(T)_{m}^{\delta} \subseteq(T)_{m}^{\alpha}$.

In Chapter 1, Section 1.3 we presented Schmerl's reflexive induction. With this tool at hand, we have access to some form of transfinite induction even if we cannot prove well-foundedness of the corresponding orderings within the base theory. Let us, by way of example, proof with detail that stronger theories yield stronger Turing progressions.

Lemma 3.2.3. Let $T$ and $U$ be theories extending $\mathrm{EA}^{+}, n<\omega$ and $\alpha \prec \Lambda$. Provably in $\mathrm{EA}^{+}$:

$$
T \subseteq U \rightarrow(T)_{n}^{\alpha} \subseteq(U)_{n}^{\alpha} .
$$

Proof. We reason informally in $\mathrm{EA}^{+}$, assume $T \subseteq U$ and use reflexive transfinite induction on $\alpha$ with the base case being trivial. Thus, for $\alpha \succ 0$, consider an arbitrary formula $\chi$ such that $\square_{(T)_{n}^{\alpha}} \chi$. Then, there is some $\delta \prec \alpha$ such that $\square_{T}\left(\operatorname{Con}_{n}\left((T)_{n}^{\delta}\right) \rightarrow \chi\right)$. By the assumption that $T \subseteq U$ together with provable monotonicity of the provability operator, we get that $\square_{(U)_{n}^{\alpha}}\left(\operatorname{Con}_{n}\left((T)_{n}^{\delta}\right) \rightarrow \chi\right)$. Moreover, we have that $\square_{(U)_{n}^{\alpha}} \operatorname{Con}_{n}\left((U)_{n}^{\delta}\right)$. Now, by the RIH we know that $\square_{\mathrm{EA}^{+}}\left((T)_{n}^{\delta} \subseteq(U)_{n}^{\delta}\right)$ so that along with Item 1 of Lemma 3.2.2 under the $\square_{\mathrm{EA}^{+}}$we obtain $\square_{(U)_{n}{ }_{n}} \operatorname{Con}_{n}\left((T)_{n}^{\delta}\right)$ and consequently $\square_{(U)_{n}^{\alpha}} \chi$.

From now on we shall include less details in our proofs that employ reflexive induction.

Lemma 3.2.4. Let $T$ be any theory extending $\mathrm{EA}^{+}$and $\sigma \in \Sigma_{n+1}^{0}$. The following principles are provable in $\mathrm{EA}^{+}$:

1. $\operatorname{Con}_{n}(T) \wedge \sigma \rightarrow \operatorname{Con}_{n}(T+\sigma)$;

2. $(T+\sigma)_{n}^{\alpha} \equiv(T)_{n}^{\alpha}+\sigma ;$ 
3. $T+\operatorname{Con}_{n}\left((T)_{n}^{\alpha}\right) \equiv(T)_{n}^{\alpha+1}$;

4. $\operatorname{Con}_{n+1}(T) \rightarrow \operatorname{Con}_{n}(T)$;

5. $(T)_{n}^{\alpha} \subseteq(T)_{n+m}^{\alpha}$.

Proof. Items 1, 3 and 4 are easy to check. The right-to-left implication of Item 2 is straightforward.

For the left-to-right implication we proceed by transfinite reflexive induction with the base case being trivial. Thus, we reason in $\mathrm{EA}^{+}$, assume as the RIH that $\square_{\mathrm{EA}^{+}} \forall \beta \prec \dot{\alpha}\left((T+\sigma)_{n}^{\beta} \subseteq(T)_{n}^{\beta}+\sigma\right)$, and let $\square_{(T+\sigma)_{n}^{\alpha}} \chi$ for some arbitrary $\chi$. Thus, $\exists \delta \prec \alpha \square_{T+\sigma}\left(\operatorname{Con}_{n}\left((T+\sigma)_{n}^{\delta}\right) \rightarrow \chi\right)$, and by monotonicity

$$
\exists \delta \prec \alpha \square_{(T)_{n}^{\alpha}+\sigma}\left(\operatorname{Con}_{n}\left((T+\sigma)_{n}^{\delta}\right) \rightarrow \chi\right) .
$$

Notice that $\square_{(T)_{n}^{\alpha}+\sigma}\left(\operatorname{Con}_{n}\left((T)_{n}^{\delta}\right) \wedge \sigma\right)$ so with the help of Item I we obtain that $\square_{(T)_{n}^{\alpha}+\sigma} \operatorname{Con}_{n}\left((T)_{n}^{\delta}+\sigma\right)$. By the RIH together with monotonicity, we get that $\square_{(T)_{n}^{\alpha}+\sigma}\left((T+\sigma)_{n}^{\delta} \subseteq(T)_{n}^{\delta}+\sigma\right)$. Combining this with Item 1 of Lemma 3.2.2 (under a box) we conclude that $\square_{(T)_{n}^{\alpha}+\sigma} \operatorname{Con}_{n}\left((T+\sigma)_{n}^{\delta}\right)$. With the help of 3.1 we finally get that $\square_{(T)_{n}^{\alpha}+\sigma} \chi$.

Item 5 tells us that Turing progressions are monotone in the consistency notion and follows from Item 4 from a straightforward reflexive transfinite induction.

Our calculus will prove all the provable relations between the Turing progressions that are considered in this dissertation. A first step and cornerstone in this study is Proposition 3.2.5 below as was proven by Schmerl in [34] for the base theory of primitive recursive arithmetic. Beklemishev generalized this to the setting of $\mathrm{EA}^{+}$with variable base theories. We cite Beklemishev's formulation here (Theorem 3 and Theorem 4 of [4]).

Proposition 3.2.5 (Fine-structure formula). Let $T$ be an axiomatizable theory which is a $\Pi_{n+2}^{0}$-axiomatized extension of $\mathrm{EA}$, then provably in $\mathrm{EA}^{+}$:

$$
\begin{array}{ll}
S_{1} \quad \forall \alpha \succ 0\left((T)_{n+m}^{\alpha} \equiv_{n}(T)_{n}^{e^{m}(\alpha)}\right) ; \\
\text { S2 } \quad \forall \beta \succ 0\left(\left((T)_{n+m}^{\beta}\right)_{n}^{\alpha} \equiv_{n}(T)_{n}^{e^{m}(\beta) \cdot(1+\alpha)}\right) .
\end{array}
$$

\section{Soundness}

In this subsection we shall show that TSC is sound for the FTP interpretation. We first present the main simple argument and will then fill out the missing details in the remainder of this subsection. 
Theorem 3.2.6 (Soundness). Given $\varphi, \psi \in \mathbb{F}_{T S C}$, if $\varphi \vdash \psi$ then

$$
\mathrm{EA}^{+} \vdash \forall x\left(\square_{T h_{\psi}}(x) \rightarrow \square_{T h_{\varphi}}(x)\right) .
$$

Proof. By induction on the length of a TSC proof of $\varphi \vdash \psi$. Thus, we see that all axioms are arithmetically sound and that all rules preserve provability.

The first three axioms and first two rules are easily seen to be arithmetically sound. In particular, Axiom $3-\left\langle n^{\alpha}\right\rangle \varphi \vdash\left\langle n^{\beta}\right\rangle \varphi$ for $\alpha \geq \beta$ - expresses the property that smooth Turing progressions are monotone in the ordinal as expressed by Item 3 of Lemma 3.2.2. On the other hand, Rule 3 -if $\varphi \vdash \psi$, then $\left\langle n^{\alpha}\right\rangle \varphi \vdash\left\langle n^{\alpha}\right\rangle \psi$ - expresses the monotonicity of smooth Turing progressions in the base theory as reflected in Lemma 3.2.3. For a proof of the soundness of the co-additivity axiom, Axiom 4, we refer to Lemma 2.6 of [2]. The remaining rule and the remaining two axioms are separately proven to be sound in the remainder of this subsection.

We will start by looking at the remaining rule. Note that in proving the soundness we may use the arithmetical counterpart of the modal axioms and rules that we already have proven to be sound. Instead of talking every time about the arithmetical counterpart of such rules and axioms, we will for the sake of presentation simply speak about the modal rules and axioms. As such, our proofs below will seem an amalgamate of modal and arithmetical reasoning.

Lemma 3.2.7. Rule 4 is sound w.r.t. the FTP interpretation.

Proof. Let us recall Rule 4:

$$
\text { If } \varphi \vdash \psi \text {, then }\left\langle n^{\alpha}\right\rangle \varphi \wedge\left\langle m^{\beta+1}\right\rangle \psi \vdash\left\langle n^{\alpha}\right\rangle\left(\varphi \wedge\left\langle m^{\beta+1}\right\rangle \psi\right) \text { for } m<n \text {. }
$$

To prove the arithmetical soundness, we assume that provably in $\mathrm{EA}^{+}$we have $T h_{\psi} \subseteq T h_{\varphi}$. Consequently $\left(T h_{\psi}+T h_{\varphi}\right) \subseteq T h_{\varphi}$ so that by Rule 3 we obtain

$$
\left(\mathrm{Th}_{\psi}+\mathrm{Th}_{\varphi}\right)_{n}^{\alpha} \subseteq\left(\mathrm{Th} \mathrm{h}_{n}^{\alpha} .\right.
$$

Also, we have the following provably in $\mathrm{EA}^{+}$:

$$
\left(\mathrm{Th}_{\varphi}+\left(\mathrm{Th}_{\psi}\right)_{m}^{\beta+1}\right)_{n}^{\alpha} \subseteq\left(\mathrm{Th}_{\varphi}+\mathrm{Th}_{\psi}+\mathrm{Con}_{m}\left(\left(\mathrm{Th}_{\psi}\right)_{m}^{\beta}\right)\right)_{n}^{\alpha},
$$

and since $\operatorname{Con}_{m}\left(\left(\mathrm{Th}_{\psi}\right)_{m}^{\beta}\right) \in \Pi_{m+1}^{0}$ and $\Pi_{m+1}^{0} \subset \Sigma_{n+1}^{0}$ we also have

$$
\left(\mathrm{Th}_{\varphi}+\mathrm{Th}_{\psi}+\operatorname{Con}_{m}\left(\left(\mathrm{Th}_{\psi}\right)_{m}^{\beta}\right)\right)_{n}^{\alpha} \subseteq\left(\mathrm{Th}_{\varphi}+\mathrm{Th}_{\psi}\right)_{n}^{\alpha}+\operatorname{Con}_{m}\left(\left(\mathrm{Th}_{\psi}\right)_{m}^{\beta}\right) \text {. }
$$

Consequently, for any formula $\chi$ we have provably in $\mathrm{EA}^{+}$that

$$
\square\left(\operatorname{Th}_{\varphi}+\left(\operatorname{Th}_{\psi}\right)_{m}^{\beta+1}\right)_{n}^{\alpha} \chi \longrightarrow \square_{\left(\operatorname{Th}_{\varphi}+\operatorname{Th}_{\psi}\right)_{n}^{\alpha}+\operatorname{Con}_{m}\left(\left(\operatorname{Th}_{\psi}\right)_{m}^{\beta}\right)} \chi
$$


Now, by the formalized deduction theorem together with 3.2 we have that for any formula $\chi$ that if $\square_{\left(\operatorname{Th}_{\varphi}+\operatorname{Th}_{\psi}\right)_{n}^{\alpha}+\operatorname{Con}_{m}\left(\left(\operatorname{Th}_{\psi}\right)_{m}^{\beta}\right)} \chi$, then

$$
\square\left(\operatorname{Th}_{\varphi}\right)_{n}^{\alpha}\left(\operatorname{Con}_{m}\left(\left(\mathrm{Th}_{\psi}\right)_{m}^{\beta}\right) \rightarrow \chi\right)
$$

whence by monotonicity $\square\left(\operatorname{Th}_{\psi}\right)_{n}^{\alpha}+\left(\mathrm{Th}_{\psi}\right)_{m}^{\beta+1}\left(\operatorname{Con}_{m}\left(\left(\mathrm{Th}_{\psi}\right)_{m}^{\beta}\right) \rightarrow \chi\right)$. In particular we get $\square\left(\mathrm{Th}_{\varphi}\right)_{n}^{\alpha}+\left(\mathrm{Th}_{\psi}\right)_{m}^{\beta+1} \chi$ as was to be shown.

We will now consider Axiom 5: $\left\langle(m+n)^{\alpha}\right\rangle \varphi \vdash\left\langle m^{e^{n}(\alpha)}\right\rangle \varphi$. It is easy to see that we can reduce the arithmetical soundness of this axiom to the arithmetical soundness of $\left\langle(n+1)^{\alpha}\right\rangle \varphi \vdash\left\langle n^{e(\alpha)}\right\rangle \varphi$. Thus, we just check the soundness of this principle with the help of the following proposition:

Proposition 3.2.8. Let $\varphi(x)$ be any arithmetical formula. By $I_{\varphi}(\bar{n})$ we denote the formula $\varphi(0) \wedge \forall x(\varphi(x) \rightarrow \varphi(s(x))) \rightarrow \varphi(\bar{n})$. For any extension $T$ of $\mathrm{EA}$, provably in $\mathrm{EA}^{+}$:

$$
\forall n \square_{T} I_{\varphi}(\bar{n}) .
$$

Proof. Sketch: We consider the proof that combines $\varphi(0)$ with $\varphi(0) \rightarrow \varphi(1)$ to obtain $\varphi(1)$ and so forth till arriving at $\varphi(\bar{n})$ and observe that the Gödel number of this proof can be exponentially bounded in the Gödel number of $\varphi$.

The following lemma states a slightly stronger principle than the one captured by Axiom 5 and thus suits the purpose of proving soundness.

Lemma 3.2.9. For any extension $\mathrm{T}$ of $\mathrm{EA}^{+}$, any ordinals $\alpha, \beta \prec \Lambda$ and natural number $n$, provably in $\mathrm{EA}^{+}$we have:

$$
\left((T)_{n}^{\beta}\right)_{n}^{e(\alpha)} \subseteq\left((T)_{n}^{\beta}\right)_{n+1}^{\alpha} .
$$

Proof. By reflexive transfinite induction on $\alpha$ with the base case being trivial. Thus, reasoning in $\mathrm{EA}^{+}$, we assume

$$
\square_{\mathrm{EA}^{+}} \forall \gamma \prec \dot{\alpha} \forall \beta\left(\left((T)_{n}^{\beta}\right)_{n}^{e(\gamma)} \subseteq\left((T)_{n}^{\beta}\right)_{n+1}^{\gamma}\right)
$$

as the RIH. We proceed by a case distinction on $\alpha$.

If $\alpha \in$ Succ, i.e., $\alpha:=\delta+1$, let $\chi$ be some arbitrary formula such that $\square\left((T)_{n}^{\beta}\right)_{n}^{e(\delta+1)} \chi$. Note that $e(\delta+1)=e(\delta) \cdot \omega$. Therefore we have

$$
\square\left((T)_{n}^{\beta}\right)_{n}^{e(\delta+1)} \chi \longrightarrow \exists m \prec \omega \square_{T}\left(\operatorname{Con}_{n}\left(\left((T)_{n}^{\beta}\right)_{n}^{e(\delta) \cdot m}\right) \rightarrow \chi\right),
$$


and by monotonicity of provability we obtain

$$
\exists m \prec \omega \square\left((T)_{n}^{\beta}\right)_{n+1}^{\delta+1}\left(\operatorname{Con}_{n}\left(\left((T)_{n}^{\beta}\right)_{n}^{e(\delta) \cdot m}\right) \rightarrow \chi\right) .
$$

Let $\theta(x):=\operatorname{Con}_{n}\left(\left((T)_{n}^{\beta}\right)_{n}^{e(\delta) \cdot \dot{x}}\right)$. We prove

$$
\forall m \prec \omega \square{ }_{\left((T)_{n}^{\beta}\right)_{n+1}^{\delta+1}} \theta(\dot{m})
$$

by applying Proposition 3.2.8. Therefore, it suffices to show

$$
\square\left((T)_{n}^{\beta}\right)_{n+1}^{\delta+1}(\theta(0) \wedge \forall x(\theta(x) \rightarrow \theta(s(x)))) .
$$

Clearly $\square\left((T)_{n}^{\beta}\right)_{n+1}^{\delta+1} \operatorname{Con}_{n}(T)$, that is, $\square\left({ }_{\left.(T)_{n}^{\beta}\right)_{n+1}^{\delta+1}} \theta(0)\right.$. We only need to check that

$$
\square\left((T)_{n}^{\beta}\right)_{n+1}^{\delta+1} \forall x(\theta(x) \rightarrow \theta(s(x))) .
$$

For this purpose, assume $\square\left((T)_{n}^{\beta}\right)_{n+1}^{\delta+1} \operatorname{Con}_{n}\left(\left((T)_{n}^{\beta}\right)_{n}^{e(\delta) \cdot m}\right)$ and set out to prove $\square\left((T)_{n}^{\beta}\right)_{n+1}^{\delta+1} \operatorname{Con}_{n}\left(\left((T)_{n}^{\beta}\right)_{n}^{e(\delta) \cdot(m+1)}\right)$. Hence, we have that

$$
\square\left((T)_{n}^{\beta}\right)_{n+1}^{\delta+1}\left(\operatorname{Con}_{n}\left(\left((T)_{n}^{\beta}\right)_{n}^{e(\delta) \cdot m}\right) \wedge \operatorname{Con}_{n+1}\left(\left((T)_{n}^{\beta}\right)_{n+1}^{\delta}\right)\right) .
$$

By Lemmas 3.2.4 and 3.2.2 we can push the small consistency inside the bigger ones and obtain

$$
\square\left((T)_{n}^{\beta}\right)_{n+1}^{\delta+1} \operatorname{Con}_{n+1}\left(\left(\left((T)_{n}^{\beta}\right)_{n}^{e(\delta) \cdot m}\right)_{n+1}^{\delta}\right) .
$$

We note that $\left((T)_{n}^{\beta}\right)_{n}^{e(\delta) \cdot m} \equiv(T)_{n}^{\beta+e(\delta) \cdot m}$ and by the RIH we have that

$$
\square\left((T)_{n}^{\beta}\right)_{n+1}^{\delta+1}\left(\left((T)_{n}^{\beta+e(\delta) \cdot m}\right)_{n}^{e(\delta)} \subseteq\left((T)_{n}^{\beta+e(\delta) \cdot m}\right)_{n+1}^{\delta}\right) .
$$

Thus, $\square\left((T)_{n}^{\beta}\right)_{n+1}^{\delta+1} \operatorname{Con}_{n+1}\left(\left((T)_{n}^{\beta+e(\delta) \cdot m}\right)_{n}^{e(\delta)}\right)$, and by monotonicity

$$
\square\left((T)_{n}^{\beta}\right)_{n+1}^{\delta+1} \operatorname{Con}_{n}\left(\left((T)_{n}^{\beta+e(\delta) \cdot m}\right)_{n}^{e(\delta)}\right) .
$$

We can observe that $\left((T)_{n}^{\beta+e(\delta) \cdot m}\right)_{n}^{e(\delta)} \equiv\left((T)_{n}^{\beta}\right)_{n}^{e(\delta) \cdot(m+1)}$. Therefore, we have that $\square\left((T)_{n}^{\beta}\right)_{n+1}^{\delta+1} \operatorname{Con}_{n}\left(\left((T)_{n}^{\beta}\right)_{n}^{e(\delta) \cdot(m+1)}\right)$ as required. 
In the case that $\alpha \in \operatorname{Lim}$ we assume $\square\left(\left((T)_{n}^{\beta}\right)_{n}^{e(\alpha)} \chi\right.$. Since $e$ is a continuous function, as before we have that

$$
\exists \delta \prec \alpha \square_{T}\left(\operatorname{Con}_{n}\left(\left((T)_{n}^{\beta}\right)_{n}^{e(\delta)}\right) \rightarrow \chi\right) .
$$

By monotonicity we obtain for this $\delta$ that

$$
\square\left((T)_{n}^{\beta}\right)_{n+1}^{\alpha}\left(\operatorname{Con}_{n}\left(\left((T)_{n}^{\beta}\right)_{n}^{e(\delta)}\right) \rightarrow \chi\right) .
$$

Since $\square\left((T)_{n}^{\beta}\right)_{n+1}^{\alpha} \operatorname{Con}_{n}\left(\left((T)_{n}^{\beta}\right)_{n+1}^{\delta}\right)$ by the RIH we get

$$
\square\left((T)_{n}^{\beta}\right)_{n+1}^{\alpha} \operatorname{Con}_{n}\left(\left((T)_{n}^{\beta}\right)_{n}^{e(\delta)}\right)
$$

so that $\square\left((T)_{n}^{\beta}\right)_{n+1}^{\alpha} \chi$.

Corollary 3.2.10. Axiom 5 is sound.

Proof. This follows directly from the previous lemma by taking $\beta=0$.

Before we are going to prove the soundness of the Schmerl axioms, we first need some observations to the extent that for sufficiently similar base theories, their corresponding Turing progressions will be similar as well.

Lemma 3.2.11. For any theories $T$ and $U$ extending EA, $0<m$ and $\alpha \prec \Lambda$, if, provably in $\mathrm{EA}^{+}, T \equiv_{n+m} U$, then

$$
\mathrm{EA}^{+} \vdash \operatorname{Con}_{n}\left((T)_{n}^{\alpha}\right) \leftrightarrow \operatorname{Con}_{n}\left((U)_{n}^{\alpha}\right) .
$$

Proof. For reasoning in $\mathrm{EA}^{+}$, suppose that $T \equiv_{n+m} U$. By reflexive transfinite induction on $\alpha$. Since base case is trivial, we check the Lemma for $\alpha \succ 0$. Thus, assume as RIH that $\square_{\mathrm{EA}^{+}} \forall \beta \prec \dot{\alpha}\left(\operatorname{Con}_{n}\left((T)_{n}^{\beta}\right) \leftrightarrow \operatorname{Con}_{n}\left((U)_{n}^{\beta}\right)\right)$.

For the left-to-right implication, assume $\operatorname{Con}_{n}\left((T)_{n}^{\alpha}\right)$ and assume for $\pi \in$ $\Pi_{n+1}^{0}, \square_{(U)_{n}^{\alpha}} \pi$. Thus, $\square_{U}\left(\operatorname{Con}_{n}\left((U)_{n}^{\delta}\right) \rightarrow \pi\right)$ for $\delta \prec \alpha$. Since $\pi \in \Pi_{n+1}^{0}$ and $n<n+m$, by assumption, $\square_{T}\left(\operatorname{Con}_{n}\left((U)_{n}^{\delta}\right) \rightarrow \pi\right)$ and by monotonicity,

$$
\square_{(T)_{n}^{\alpha}}\left(\operatorname{Con}_{n}\left((U)_{n}^{\delta}\right) \rightarrow \pi\right) .
$$

Observe that $\square_{(T)_{n}^{\alpha}} \operatorname{Con}_{n}\left((T)_{n}^{\delta}\right)$, and since by monotonicity the RIH holds provably in $(T)_{n}^{\alpha}$, we conclude that $\square_{(T)_{n}^{\alpha}} \pi$. For the other other implication we reason analogously. 
With this previous result, we can establish the following corollary about the conservativity between Turing progressions built up from sufficiently similar base theories.

Corollary 3.2.12. For any theories $T$ and $U$ extending $\mathrm{EA}$, any $m>0$ and any ordinal $\alpha<\Lambda$,

$$
\mathrm{EA}^{+} \vdash T \equiv_{n+m} U \Longrightarrow \mathrm{EA}^{+} \vdash(T)_{n}^{\alpha} \equiv_{n}(U)_{n}^{\alpha} .
$$

Proof. For reasoning in $\mathrm{EA}^{+}$, suppose that $T \equiv_{n+m} U$. Let $\pi \in \Pi_{n+1}^{0}$ and assume $\square_{(T)_{n}^{\alpha}} \pi$ for some arbitrary $\alpha$. Thus,

$$
\exists \delta \prec \alpha \square_{T}\left(\operatorname{Con}_{n}\left((T)_{n}^{\delta}\right) \rightarrow \pi\right) .
$$

By assumption together with monotonicity, we have that

$$
\square_{(U)_{n}^{\alpha}}\left(\left(\operatorname{Con}_{n}\left((T)_{n}^{\delta}\right) \rightarrow \pi\right) \wedge \operatorname{Con}_{n}\left((U)_{n}^{\delta}\right)\right) .
$$

Therefore, by previous lemma, $\square_{(U)_{n}^{\alpha}} \operatorname{Con}_{n}\left((T)_{n}^{\delta}\right)$ and so $\square_{(U)_{n}^{\alpha}} \pi$. For the other inclusion we reason analogously.

We can now prove that the smallest term of an MNF captures all the small consistency strength that is implied by the entire MNF.

Lemma 3.2.13. Let $\psi:=\left\langle n_{0}^{\alpha_{0}}\right\rangle \top \wedge \ldots \wedge\left\langle n_{k}^{\alpha_{k}}\right\rangle \top \in M N F$. We then have that provably in $\mathrm{EA}^{+}$:

$$
T h_{\psi} \equiv_{n_{0}}\left(\mathrm{EA}^{+}\right)_{n_{0}}^{\alpha_{0}} .
$$

Proof. By induction on $k$ with base case being trivial. For the inductive step, let $\psi^{\prime}:=\left\langle n_{1}^{\alpha_{1}}\right\rangle \top \wedge \ldots \wedge\left\langle n_{k}^{\alpha_{k}}\right\rangle \top \in \mathrm{MNF}$ and $\psi:=\left\langle n_{0}^{\alpha_{0}}\right\rangle \top \wedge \psi^{\prime} \in \mathrm{MNF}$. Notice that the right-to-left inclusion is straightforward so we just check the left-to-right direction. Reasoning in $\mathrm{EA}^{+}$, suppose that $\square_{\mathrm{Th}_{\psi}} \pi$ for some $\pi \in \Pi_{n_{0}+1}^{0}$. Thus, $\square_{\mathrm{Th}_{\psi^{\prime}}}\left(\operatorname{Con}_{n_{0}}\left(\left(\mathrm{EA}^{+}\right)_{n_{0}}^{\delta}\right) \rightarrow \pi\right)$ for some $\delta \prec \alpha_{0}$. Notice that

$$
\left(\mathrm{Con}_{n_{0}}\left(\left(\mathrm{EA}^{+}\right)_{n_{0}}^{\delta}\right) \rightarrow \pi\right) \in \Pi_{n_{0}+2}^{0}
$$

whence also in $\Pi_{n_{1}+1}^{0}$ so that by the external induction on $k$ we get that $\square_{\left(\mathrm{EA}^{+}\right)_{n_{1}}^{\alpha_{1}}}\left(\mathrm{Con}_{n_{0}}\left(\left(\mathrm{EA}^{+}\right)_{n_{0}}^{\delta}\right) \rightarrow \pi\right)$. By monotonicity it follows that

$$
\square_{\left(\left(\mathrm{EA}^{+}\right)_{n_{1}}^{\alpha_{1}}\right)_{n_{0}}^{\beta_{0}}}\left(\operatorname{Con}_{n_{0}}\left(\left(\mathrm{EA}^{+}\right)_{n_{0}}^{\delta}\right) \rightarrow \pi\right)
$$

for $\beta_{0}$ so that $\alpha_{0}=e^{n_{1}-n_{0}}\left(\alpha_{1}\right) \cdot\left(1+\beta_{0}\right)$. Notice that by Schmerl's finestructure formula (Item 3.2.5 of Proposition 3.2.5) together with the facts that 
$\delta \prec \alpha_{0}$ and $\operatorname{Con}_{n_{0}}\left(\left(\mathrm{EA}^{+}\right)_{n_{0}}^{\delta}\right) \in \Pi_{n_{0}+1}^{0}$, we get that $\square\left(\left(\mathrm{EA}^{+}\right)_{n_{1}}^{\alpha_{1}}\right)_{n_{0}}^{\beta_{0}} \operatorname{Con}_{n_{0}}\left(\left(\mathrm{EA}^{+}\right)_{n_{0}}^{\delta}\right)$ and so, $\square\left(\left(\mathrm{EA}^{+}\right)_{n_{1}}^{\alpha_{1}}\right)_{n_{0}}^{\beta_{0}} \pi$. Applying Schmerl's fine-structure formula again we get that $\square\left(\mathrm{EA}^{+}\right)_{n_{0}}^{\alpha_{0}} \pi$.

The lemma can be easily generalized to conclude that initial truncations of an MNF provide optimal approximations of the entire MNF.

Lemma 3.2.14. Let $\psi:=\left\langle n_{0}^{\alpha_{0}}\right\rangle \top \wedge \ldots \wedge\left\langle n_{k}^{\alpha_{k}}\right\rangle \top \in M N F$. We then have for any $j \leq k$ that provably in $\mathrm{EA}^{+}$:

$$
T h_{\psi} \equiv_{n_{j}}\left(\mathrm{EA}^{+}\right)_{n_{0}}^{\alpha_{0}} \wedge \ldots \wedge\left(\mathrm{EA}^{+}\right)_{n_{j}}^{\alpha_{j}}
$$

Proof. The right-to-left implication is straightforward. For the other implication, we make the following case distinction on $j$. If $j=0$, then it follows from Lemma 3.2.13. If $j \neq 0$, for reasoning within $\mathrm{EA}^{+}$, let $\pi \in \Pi_{n_{j}+1}^{0}$ such that $\square \mathrm{Th}_{\psi} \pi$. By the formalized deduction theorem we get that

$$
\square_{\left(\mathrm{EA}^{+}\right)_{n_{j}}^{\alpha_{j}}+\ldots+\left(\mathrm{EA}^{+}\right)_{n_{k}}^{\alpha_{k}}}\left(\bigwedge_{0 \leq i<j} \operatorname{Con}_{n_{i}}\left(\left(\mathrm{EA}^{+}\right)_{n_{i}}^{\beta_{i}}\right) \rightarrow \pi\right)
$$

for some sequence of $\beta_{i} \prec \alpha_{i}$. Notice that each $\operatorname{Con}_{n_{i}}\left(\left(\mathrm{EA}^{+}\right)_{n_{i}}^{\beta_{i}}\right) \in \Pi_{n_{i}+1}^{0}$ with $n_{i}<n_{j}$. Therefore, $\left(\Lambda_{0 \leq i<j^{\prime}} \operatorname{Con}_{n_{i}}\left(\left(\mathrm{EA}^{+}\right)_{n_{i}}^{\beta_{i}}\right) \rightarrow \pi\right) \in \Pi_{n_{j}+1}^{0}$.

Also, notice that $\left\langle n_{j}^{\alpha_{j}}\right\rangle \top \wedge \ldots \wedge\left\langle n_{k}^{\alpha_{k}}\right\rangle \top \in$ MNF. Thus, applying Lemma 3.2.13, we obtain that

$$
\square_{\left(\mathrm{EA}^{+}\right)_{n_{j}}^{\alpha_{j}}}\left(\bigwedge_{0 \leq i<j} \operatorname{Con}_{n_{i}}\left(\left(\mathrm{EA}^{+}\right)_{n_{i}}^{\beta_{i}}\right) \rightarrow \pi\right)
$$

and by monotonicity,

$$
\square_{\left(\mathrm{EA}^{+}\right)_{n_{0}}^{\alpha_{0}}+\ldots+\left(\mathrm{EA}^{+}\right)_{n_{j}}^{\alpha_{j}}}\left(\bigwedge_{0 \leq i<j} \operatorname{Con}_{n_{i}}\left(\left(\mathrm{EA}^{+}\right)_{n_{i}}^{\beta_{i}}\right) \rightarrow \pi\right) .
$$

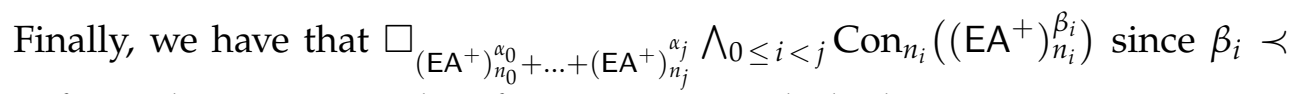
$\alpha_{i}$ for each $i, 0 \leq i<j$. Therefore, we can conclude that

$$
\square\left(\mathrm{EA}^{+}\right)_{n_{0}}^{\alpha_{0}}+\ldots+\left(\mathrm{EA}^{+}\right)_{n_{j}}^{\alpha_{j}} \pi
$$

We are now ready to prove the soundness of our more complicated axiom. 
Lemma 3.2.15. Schmerl's axioms-Axiom 6-is sound. That is,

$$
\left\langle n^{\alpha}\right\rangle\left(\left\langle n_{0}^{\alpha_{0}}\right\rangle \top \wedge \psi\right) \equiv\left\langle n^{e^{n_{0}-n}\left(\alpha_{0}\right) \cdot(1+\alpha)}\right\rangle \top \wedge\left\langle n_{0}^{\alpha_{0}}\right\rangle \top \wedge \psi
$$

for $n<n_{0}$ and $\left\langle n_{0}^{\alpha_{0}}\right\rangle \top \wedge \psi \in M N F$.

Proof. Let $\psi^{\prime}:=\left\langle n_{0}^{\alpha_{0}}\right\rangle \top \wedge \psi$. Thus, by the previous lemma we have that

$$
\mathrm{EA}^{+} \vdash \mathrm{Th}_{\psi^{\prime}} \equiv_{n_{0}}\left(\mathrm{EA}^{+}\right)_{n_{0}}^{\alpha_{0}}
$$

and then, since $n<n_{0}$, by Corollary 3.2.12 we get that

$$
\mathrm{EA}^{+} \vdash\left(\mathrm{Th}_{\psi^{\prime}}\right)_{n}^{\alpha} \equiv_{n}\left(\left(\mathrm{EA}^{+}\right)_{n_{0}}^{\alpha_{0}}\right)_{n}^{\alpha} .
$$

Thus, applying Schmerl's fine-structure formula (Proposition 3.2.5, Item 3.2.5), we have that $\mathrm{EA}^{+} \vdash\left(\mathrm{Th}_{\psi^{\prime}}\right)_{n}^{\alpha} \equiv_{n}\left(\mathrm{EA}^{+}\right)_{n}^{e^{n_{0}-n}\left(\alpha_{0}\right) \cdot(1+\alpha)}$.

To check the soundness of Axioms 6, we need to see that provably in $\mathrm{EA}^{+}:\left(\mathrm{Th}_{\psi^{\prime}}\right)_{n}^{\alpha} \equiv\left(\mathrm{EA}^{+}\right)_{n}^{e^{n_{0}-n}\left(\alpha_{0}\right) \cdot(1+\alpha)}+\mathrm{Th}_{\psi^{\prime}}$. For the right-to-left inclusion, suppose that $\square\left(\mathrm{EA}^{+}\right)_{n}^{e^{n_{0}-n}\left(\alpha_{0}\right) \cdot(1+\alpha)}+\mathrm{Th}_{\psi^{\prime}} \varphi$ for some formula $\varphi$ of arbitrary complexity. Therefore, by the formalized deduction theorem,

$$
\exists \delta \prec e^{n_{0}-n}\left(\alpha_{0}\right) \cdot(1+\alpha) \square_{\mathrm{Th}_{\psi^{\prime}}}\left(\operatorname{Con}_{n}\left(\left(\mathrm{EA}^{+}\right)_{n}^{\delta}\right) \rightarrow \varphi\right) .
$$

Hence, by monotonicity, $\square\left(\mathrm{Th}_{\psi^{\prime}}\right)_{n}^{\alpha}\left(\operatorname{Con}_{n}\left(\left(\mathrm{EA}^{+}\right)_{n}^{\delta}\right) \rightarrow \varphi\right)$. Since we have that provably in $\mathrm{EA}^{+},\left(\mathrm{Th}_{\psi^{\prime}}\right)_{n}^{\alpha} \equiv_{n}\left(\mathrm{EA}^{+}\right)_{n}^{e^{n_{0}-n}\left(\alpha_{0}\right) \cdot(1+\alpha)}$, that $\operatorname{Con}_{n}\left(\left(\mathrm{EA}^{+}\right)_{n}^{\delta}\right) \in$ $\Pi_{n+1}^{0}$ and that $\delta \prec e^{n_{0}-n}\left(\alpha_{0}\right) \cdot(1+\alpha)$, we get that $\square\left(\operatorname{Th}_{\psi^{\prime}}\right)_{n}^{\alpha} \operatorname{Con}_{n}\left(\left(\mathrm{EA}^{+}\right)_{n}^{\delta}\right)$ and so, $\square\left(\mathrm{Th}_{\psi^{\prime}}\right)_{n}^{\alpha} \varphi$.

For the left-to-right inclusion, assume $\square\left(\mathrm{Th}_{\psi^{\prime}}\right)_{n}{ }_{n} \varphi$ for some $\varphi$. Then, by the formalized deduction theorem, $\square_{\mathrm{Th}_{\psi^{\prime}}}\left(\operatorname{Con}_{n}\left(\left(\mathrm{Th}_{\psi^{\prime}}\right)_{n}^{\delta}\right) \rightarrow \varphi\right)$ for some $\delta \prec \alpha$ and by monotonicity, $\square\left(\mathrm{EA}^{+}\right)_{n}^{e^{n_{0}-n}\left(\alpha_{0}\right) \cdot(1+\alpha)}+\mathrm{Th}_{\psi^{\prime}}\left(\operatorname{Con}_{n}\left(\left(\mathrm{Th}_{\psi^{\prime}}\right)_{n}^{\delta}\right) \rightarrow \varphi\right)$. Since we have that $\operatorname{Con}_{n}\left(\left(\operatorname{Th}_{\psi^{\prime}}\right)_{n}^{\delta}\right) \in \Pi_{n+1}^{0}$, that $\square_{\left(\mathrm{Th}_{\psi^{\prime}}\right)_{n}} \operatorname{Con}_{n}\left(\left(\mathrm{Th}_{\psi^{\prime}}\right)_{n}^{\delta}\right)$ and that provably in $\mathrm{EA}^{+}$,

$$
\left(\mathrm{Th}_{\psi^{\prime}}\right)_{n}^{\alpha} \equiv_{n}\left(\mathrm{EA}^{+}\right)_{n}^{e^{n_{0}-n}\left(\alpha_{0}\right) \cdot(1+\alpha)}
$$

and

$$
\left(\mathrm{EA}^{+}\right)_{n}^{e^{n_{0}-n}\left(\alpha_{0}\right) \cdot(1+\alpha)} \subseteq\left(\mathrm{EA}^{+}\right)_{n}^{e^{n_{0}-n}\left(\alpha_{0}\right) \cdot(1+\alpha)}+\mathrm{Th}_{\psi^{\prime}},
$$

then, we have that $\square\left(\mathrm{EA}^{+}\right)_{n}^{e^{n_{0}-n}\left(\alpha_{0}\right) \cdot(1+\alpha)}+\operatorname{Th}_{\psi^{\prime}} \operatorname{Con}_{n}\left(\left(\mathrm{Th}_{\psi^{\prime}}\right)_{n}^{\delta}\right)$ and this way, we get that $\square\left(\mathrm{EA}^{+}\right)_{n}^{e^{n_{0}-n}\left(\alpha_{0}\right) \cdot(1+\alpha)}+\mathrm{Th}_{\psi^{\prime}} \varphi$. This concludes the proof of the lemma. 


\subsection{Completeness of TSC}

In this section, we shall prove the uniqueness of MNF's (Theorem 3.3.6) together with some important facts that characterize the derivability in TSC. With these results we shall show the completeness of our system in Theorem $3 \cdot 3 \cdot 13$.

\section{Conservativity and uniqueness of MNF's}

The following corollary is a result of combining arithmetical soundness of TSC together with Gödel's second incompleteness theorem.

Corollary 3.3.1. For any formula $\varphi \in \mathbb{F}_{T S C}, n<\omega$ and $0<\alpha<\Lambda$, the sequent $\varphi \vdash\left\langle n^{\alpha}\right\rangle \varphi$ is not derivable in TSC.

As we shall see, this corollary is the only tool needed to obtain the required results on non-derivability. Many of the results that we will prove below can be proven in a shorter fashion using the soundness theorem. Moreover, Corollary 3.3.1 can also be obtained via relational semantics as we will show in the following chapter.

The following result is an application of Corollary 3.3.1.

Proposition 3.3.2. Let $\psi:=\left\langle n_{0}^{\alpha_{0}}\right\rangle \top \wedge \ldots \wedge\left\langle n_{k}^{\alpha_{k}}\right\rangle \top \in M N F$. For any $i$ with $0 \leq i \leq k$ the sequent $\psi \vdash\left\langle n_{i}^{\alpha_{i}+1}\right\rangle \top$ is not derivable.

Proof. If $k=0$ then the result follows directly from a combination of Axiom 4 and Corollary 3.3.1. For $k>0$, assume $\psi \vdash\left\langle n_{i}^{\alpha_{i}+1}\right\rangle \top$ and reason as follows:

$$
\begin{aligned}
\left\langle n_{i}^{1}\right\rangle \psi & \vdash\left\langle n_{0}^{\alpha_{0}+1}\right\rangle \top \wedge \ldots \wedge\left\langle n_{i}^{1}\right\rangle\left\langle n_{i}^{\alpha_{i}+1}\right\rangle \top \text { by (PSI) and monotonicity; } \\
& \vdash\left\langle n_{i}^{\alpha_{i}+2}\right\rangle\left\langle n_{0}^{\alpha_{0}+1}\right\rangle \top \wedge \ldots \wedge\left\langle n_{i}^{2}\right\rangle\left\langle n_{i}^{\alpha_{i}}\right\rangle \top \text { by Axiom } 4 \text { and } \\
& \text { Proposition } 2.2 .2 \text {, Item } 4 ; \\
& \vdash\left\langle n_{i}^{2}\right\rangle\left\langle n_{0}^{\alpha_{0}+1}\right\rangle \top \wedge \ldots \wedge\left\langle n_{i}^{2}\right\rangle\left\langle n_{i}^{\alpha_{i}}\right\rangle \top \text { by monotonicity; } \\
& \vdash\left\langle n_{0}^{\alpha_{0}+e^{n_{i}-n_{0}}(2)}\right\rangle \top \wedge \ldots \wedge\left\langle n_{i}^{2}\right\rangle\left\langle n_{i}^{\alpha_{i}}\right\rangle \top \text { by Axioms } 5 \text { and } 4 .
\end{aligned}
$$

Therefore, in case $i=k$, by (PSI) we get that $\left\langle n_{i}^{1}\right\rangle \psi \vdash\left\langle n_{i}^{2}\right\rangle \psi$ which by means of Corollary 3.3.1 makes the sequent $\psi \vdash\left\langle n_{k}^{\alpha_{k}+1}\right\rangle \top$ not derivable. If 
$i<k$, we can make the following observation:

$$
\begin{aligned}
\left\langle n_{i}^{1}\right\rangle \psi & \vdash\left\langle n_{i}^{1}\right\rangle\left(\left\langle n_{i}^{\alpha_{i}+1}\right\rangle \top \wedge\left\langle n_{i+1}^{\alpha_{i+1}}\right\rangle \top\right) ; \\
& \vdash\left\langle n_{i}^{1}\right\rangle\left(\left\langle n_{i+1}^{\alpha_{i+1}}\right\rangle\left\langle n_{i}^{\alpha_{i}+1}\right\rangle \top \wedge\left\langle n_{i+1}^{\alpha_{i+1}}\right\rangle \top\right) \\
& \\
& \vdash\left\langle n_{i}^{1}\right\rangle\left(\left\langle n_{i}^{\alpha_{i}+e^{n_{i+1}-n_{i}}\left(\alpha_{i+1}\right)}\right\rangle \top \wedge\left\langle n_{i+1}^{\alpha_{i+1}}\right\rangle \top\right) \\
& \quad \text { by Pxiom } 5 \text { and Axiom 4; } \\
& \vdash\left\langle n_{i}^{\alpha_{i}+e^{n_{i+1}-n_{i}}\left(\alpha_{i+1}\right)+1}\right\rangle \top \wedge\left\langle n_{i+1}^{\alpha_{i+1}}\right\rangle \top \text { by Axiom 4; } \\
& \vdash\left\langle n_{i+1}^{\alpha_{i+1}}\right\rangle\left\langle n_{i}^{\alpha_{i}+e^{n_{i+1}-n_{i}}\left(\alpha_{i+1}\right)+1}\right\rangle \top \wedge\left\langle n_{i+1}^{\alpha_{i+1}}\right\rangle \top \\
& \vdash\left\langle n_{i}^{\alpha_{i}+e^{n_{i+1}-n_{i}}\left(\alpha_{i+1}\right) \cdot 2}\right\rangle \top \wedge\left\langle n_{i+1}^{\alpha_{i+1}}\right\rangle \top \text { by Axiom } 5 \text { and Axiom 4; } \\
& \vdash\left\langle n_{i}^{\alpha_{i}+e^{n_{i+1}-n_{i}}\left(\alpha_{i+1}\right) \cdot 2}\right\rangle \top \wedge\left\langle n_{i+1}^{\alpha_{i+1}}\right\rangle \top \wedge \ldots \wedge\left\langle n_{k}^{\alpha_{k}}\right\rangle \top \\
& \vdash\left\langle n_{i}^{2}\right\rangle\left(\left\langle n_{i}^{\alpha_{i}}\right\rangle \top \wedge \ldots \wedge\left\langle n_{k}^{\alpha_{k}}\right\rangle \top\right) \text { by (PS2). }
\end{aligned}
$$

Combining this result with previous reasoning we have that

$$
\left\langle n_{i}^{1}\right\rangle \psi \vdash\left\langle n_{0}^{\alpha_{0}+e^{n_{i}-n}(2)}\right\rangle \top \wedge \ldots \wedge\left\langle n_{i}^{2}\right\rangle\left(\left\langle n_{i}^{\alpha_{i}}\right\rangle \top \wedge \ldots \wedge\left\langle n_{k}^{\alpha_{k}}\right\rangle \top\right)
$$

and then, by (PSI) we get that $\left\langle n_{i}^{1}\right\rangle \psi \vdash\left\langle n_{i}^{2}\right\rangle \psi$. Hence, by Corollary 3.3.1 we obtain that the sequent $\psi \vdash\left\langle n_{i}^{\alpha_{i}+1}\right\rangle \top$ is not derivable.

The next proposition tells us in a sense that we are justified to not necessarily enlist consecutive modalities in MNF's. For the sake of clarity let us introduce the following definition.

Definition 3.3.3. Let $\psi:=\left\langle n_{0}^{\alpha_{0}}\right\rangle \top \wedge \ldots \wedge\left\langle n_{k}^{\alpha_{k}}\right\rangle \top \in M N F$ and $n \leq n_{k}$. By $\mu(n, \psi)$ we denote the least upper bound of $n$ in $N-\bmod (\psi)$ defined as:

$$
\mu(n, \psi)=\min \{m \in N-\bmod (\psi): m \geq n\} .
$$

Proposition 3.3.4. Let $\psi:=\left\langle n_{0}^{\alpha_{0}}\right\rangle \top \wedge \ldots \wedge\left\langle n_{k}^{\alpha_{k}}\right\rangle \top \in M N F$. For any $n \leq n_{k}$ and $\mu(n, \psi)=n_{J}$, the following rules are derivable in TSC:

(B1) If $\psi \vdash\left\langle m^{\beta}\right\rangle \top$ for $m>n_{k}$ and $\beta>0$, then $\left\langle n_{k}^{1}\right\rangle \psi \vdash\left\langle n_{k}^{e^{m-n_{k}}(\beta)}\right\rangle \psi$;

(B2) If $\psi \vdash\left\langle n^{\gamma}\right\rangle \top$ for $\gamma>e^{n_{J}-n}\left(\alpha_{J}\right)$, then $\left\langle n_{J}^{1}\right\rangle \psi \vdash\left\langle n_{J}^{2}\right\rangle \psi$;

Proof. For a proof of (B1), suppose that $\psi \vdash\left\langle m^{\beta}\right\rangle \top$ for some $m>n_{k}$. Thus, we reason as follows: 


$$
\begin{aligned}
\left\langle n_{k}^{1}\right\rangle \psi & \vdash\left\langle n_{k}^{1}\right\rangle\left(\left\langle n_{0}^{\alpha_{0}}\right\rangle \top \wedge \ldots \wedge\left\langle n_{k}^{\alpha_{k}}\right\rangle \top\right) \wedge\left\langle m^{\beta}\right\rangle \top ; \\
& \vdash\left\langle n_{0}^{\alpha_{0}+e^{n_{k}-n_{0}}(1)}\right\rangle \top \wedge \ldots \wedge\left\langle n_{k}^{1}\right\rangle\left\langle n_{k}^{\alpha_{k}}\right\rangle \top \wedge\left\langle m^{\beta}\right\rangle \top \text { by (PSI) } ; \\
& \vdash\left\langle n_{0}^{\alpha_{0}+e^{n_{k}-n_{0}}(1)}\right\rangle \top \wedge \ldots \wedge\left\langle n_{k}^{\alpha_{k}+1}\right\rangle \top \wedge\left\langle m^{\beta}\right\rangle \top \text { by Axiom 4; } \\
& \vdash\left\langle n_{0}^{\alpha_{0}+1}\right\rangle \top \wedge \ldots \wedge\left\langle n_{k}^{\alpha_{k}+1}\right\rangle \top \wedge\left\langle m^{\beta}\right\rangle \top \text { by Axiom 3; }
\end{aligned}
$$

Notice that by Proposition 2.2.2, Item 4:

$$
\begin{aligned}
\left\langle n_{0}^{\alpha_{0}+1}\right\rangle \top \wedge \ldots \wedge\left\langle n_{k}^{\alpha_{k}+1}\right\rangle \top & \wedge\left\langle m^{\beta}\right\rangle \top \vdash \\
& \left\langle n_{0}^{\alpha_{0}+1}\right\rangle \top \wedge \ldots \wedge\left\langle m^{\beta}\right\rangle\left\langle n_{k}^{\alpha_{k}+1}\right\rangle \top \wedge\left\langle m^{\beta}\right\rangle \top .
\end{aligned}
$$

Thus, by a combination of Axioms 5 and 4 together with the previous fact we obtain that $\left\langle n_{k}^{1}\right\rangle \psi \vdash\left\langle n_{0}^{\alpha_{0}+1}\right\rangle \top \wedge \ldots \wedge\left\langle n_{k}^{\alpha_{k}+e^{m-n_{k}}(\beta)}\right\rangle \top \wedge\left\langle m^{\beta}\right\rangle \top$. Applying successively Proposition 2.2.2, Item 4 we get that

$$
\left\langle n_{k}^{1}\right\rangle \psi \vdash\left\langle n_{k}^{\alpha_{k}+e^{m-n_{k}}(\beta)}\right\rangle\left\langle n_{0}^{\alpha_{0}+1}\right\rangle \top \wedge \ldots \wedge\left\langle n_{k}^{\alpha_{k}+e^{m-n_{k}}(\beta)}\right\rangle \top \wedge\left\langle m^{\beta}\right\rangle \top,
$$

and combining Axioms 5 and 4 :

$$
\left\langle n_{k}^{1}\right\rangle \psi \vdash\left\langle n_{0}^{\alpha_{0}+e^{n_{k}-n_{0}}\left(\alpha_{k}+e^{m-n_{k}}(\beta)\right)}\right\rangle \top \wedge \ldots \wedge\left\langle n_{k}^{\alpha_{k}+e^{m-n_{k}}(\beta)}\right\rangle \top
$$

and so, by monotonicity

$$
\left\langle n_{k}^{1}\right\rangle \psi \vdash\left\langle n_{0}^{\alpha_{0}+e^{n_{k}-n_{0}}\left(e^{m-n_{k}}(\beta)\right)}\right\rangle \top \wedge \ldots \wedge\left\langle n_{k}^{\alpha_{k}+e^{m-n_{k}}(\beta)}\right\rangle \top .
$$

Finally, by successively applying (PSI) we get that $\left\langle n_{k}^{1}\right\rangle \psi \vdash\left\langle n_{k}^{e^{m-n_{k}}(\beta)}\right\rangle \psi$. Since $\beta>0$ and $m>n_{k}$ we see that $e^{m-n_{k}}(\beta) \in \lim$ so that $1+e^{m-n_{k}}(\beta)=$ $e^{m-n_{k}}(\beta)$ whence, by Axiom $4,\left\langle n_{k}^{e^{m-n_{k}}(\beta)}\right\rangle \psi \equiv\left\langle n_{k}^{e^{m-n_{k}}(\beta)}\right\rangle\left\langle n_{k}^{1}\right\rangle \psi$ and so,

$$
\left\langle n_{k}^{1}\right\rangle \psi \vdash\left\langle n_{k}^{e^{m-n_{k}}(\beta)}\right\rangle\left\langle n_{k}^{1}\right\rangle \psi
$$

As pointed out, this yields a contradiction with Gödel II as reflected in Corollary 3.3.1.

As for (B2), for $n=n_{J}$ the rule is trivially admissible since the antecedent is not derivable because of Proposition 3.3.2. Thus, we just check the case where $n<n_{J}$.

Assume $\psi \vdash\left\langle n^{\gamma}\right\rangle \top$ for some $\gamma>e^{n_{J}-n}\left(\alpha_{J}\right)$. Therefore, by Axiom 3 we get that $\psi \vdash\left\langle n^{e^{n_{J}-n}\left(\alpha_{J}\right)+1}\right\rangle \top \wedge\left\langle n_{J}^{\alpha_{J}}\right\rangle \top$ and so, by Proposition 2.2.2, Item 4,

$$
\psi \vdash\left\langle n_{J}^{\alpha_{J}}\right\rangle\left\langle n^{e^{n} J^{-n}\left(\alpha_{J}\right)+1}\right\rangle \top .
$$


By a combination of Axioms 5 and $4, \psi \vdash\left\langle n^{e^{n} J^{-n}\left(\alpha_{J}\right) \cdot 2}\right\rangle \top$ and hence,

$$
\left\langle n^{1}\right\rangle \psi \vdash\left\langle n^{e^{n_{J}-n}}\left(\alpha_{J}\right) \cdot 2+1\right\rangle \top \wedge\left\langle n_{J}^{\alpha_{J}}\right\rangle \top .
$$

By Proposition 2.2.2, Item 4, combined with Axioms 5 and 4,

$$
\left\langle n^{1}\right\rangle \psi \vdash\left\langle n^{e^{n-n}\left(\alpha_{J}\right) \cdot 3}\right\rangle \top .
$$

By (PS1) and Axiom 6, we have that

$$
\left\langle n^{1}\right\rangle \psi \vdash\left\langle n_{0}^{\alpha_{0}+e^{n-n_{0}}(1)}\right\rangle \top \wedge \ldots \wedge\left\langle n^{e^{n_{J}-n}\left(\alpha_{J}\right) \cdot 2}\right\rangle \top \wedge\left\langle n_{J}^{\alpha_{J}}\right\rangle \top \wedge \ldots \wedge\left\langle n_{k}^{\alpha_{k}}\right\rangle \top .
$$

This combined with (3.5) give us that

$$
\left\langle n^{1}\right\rangle \psi \vdash\left\langle n_{0}^{\alpha_{0}+e^{n-n_{0}}(1)}\right\rangle \top \wedge \ldots \wedge\left\langle n^{e^{n_{J}-n}\left(\alpha_{J}\right) \cdot 3}\right\rangle \top \wedge\left\langle n_{J}^{\alpha_{J}}\right\rangle \top \wedge \ldots \wedge\left\langle n_{k}^{\alpha_{k}}\right\rangle \top .
$$

Now we can reason as follows:

$$
\begin{aligned}
& \left.\left\langle n^{1}\right\rangle \psi \vdash\left\langle n_{0}^{\alpha_{0}+e^{n-n_{0}}(1)}\right\rangle \top \wedge \ldots \wedge n^{e^{n} J-n}\left(\alpha_{J}\right) \cdot 3\right\rangle \top \wedge \\
& \left\langle n_{J}^{\alpha_{J}}\right\rangle \top \wedge \ldots \wedge\left\langle n_{k}^{\alpha_{k}}\right\rangle \top \text {; } \\
& \vdash\left\langle n_{0}^{\alpha_{0}+1}\right\rangle \top \wedge \ldots \wedge\left\langle n^{e^{n_{J}-n}\left(\alpha_{J}\right) \cdot 3}\right\rangle \top \wedge \\
& \left\langle n_{J}^{\alpha_{J}}\right\rangle \top \wedge \ldots \wedge\left\langle n_{k}^{\alpha_{k}}\right\rangle \top \text { by monotonicity; } \\
& \vdash\left\langle n^{e^{n} J-n}\left(\alpha_{J}\right) \cdot 3\right\rangle\left\langle n_{0}^{\alpha_{0}+1}\right\rangle \top \wedge \ldots \wedge\left\langle n^{e^{n-n}\left(\alpha_{J}\right) \cdot 3}\right\rangle \top \wedge \\
& \left\langle n_{J}^{\alpha_{J}}\right\rangle \top \wedge \ldots \wedge\left\langle n_{k}^{\alpha_{k}}\right\rangle \top \text { by Proposition 2.2.2, Item 4; } \\
& \vdash\left\langle n^{2}\right\rangle\left\langle n_{0}^{\alpha_{0}+1}\right\rangle \top \wedge \ldots \wedge\left\langle n^{e^{n} J^{-n}\left(\alpha_{J}\right) \cdot 3}\right\rangle \top \wedge \\
& \left\langle n_{J}^{\alpha_{J}}\right\rangle \top \wedge \ldots \wedge\left\langle n_{k}^{\alpha_{k}}\right\rangle \top \text { by monotonicity; } \\
& \vdash\left\langle n^{2}\right\rangle\left\langle n_{0}^{\alpha_{0}+1}\right\rangle \top \wedge \ldots \wedge \\
& \left\langle n^{2}\right\rangle\left(\left\langle n_{J}^{\alpha_{J}}\right\rangle \top \wedge \ldots \wedge\left\langle n_{k}^{\alpha_{k}}\right\rangle \top\right) \text { by Axiom 6; } \\
& \vdash\left\langle n_{0}^{\alpha_{0}+e^{n-n_{0}}(2)}\right\rangle \top \wedge \ldots \wedge \\
& \left\langle n^{2}\right\rangle\left(\left\langle n_{J}^{\alpha_{J}}\right\rangle \top \wedge \ldots \wedge\left\langle n_{k}^{\alpha_{k}}\right\rangle \top\right) \text { by Axioms } 5 \text { and } 4 ; \\
& \vdash\left\langle n^{2}\right\rangle\left(\left\langle n_{0}^{\alpha_{0}}\right\rangle \top \wedge \ldots \wedge\left\langle n_{J}^{\alpha_{J}}\right\rangle \top \wedge \ldots \wedge\left\langle n_{k}^{\alpha_{k}}\right\rangle \top\right) \text { by (PSI); } \\
& \vdash\left\langle n^{2}\right\rangle \psi \text {. }
\end{aligned}
$$

We may observe that this result yields a contradiction with Gödel II, that is, Corollary 3.3.1. Hence, we obtain that if $\psi \vdash\left\langle n^{\gamma}\right\rangle \top$, then $\gamma \leq e^{n_{J}-n}\left(\alpha_{J}\right)$.

The following corollary gathers some immediate consequences of Rules (B1) and (B2) together with Corollary 3.3.1. 
Corollary 3.3.5. Let $\psi:=\left\langle n_{0}^{\alpha_{0}}\right\rangle \top \wedge \ldots \wedge\left\langle n_{k}^{\alpha_{k}}\right\rangle \top \in M N F, n \leq n_{k}$ and $\mu(n, \psi)=$ $n_{J}$

1. If $\psi \vdash\left\langle m^{\beta}\right\rangle \top$ for some $\beta>0$, then $m \leq n_{k}$;

2. If $\psi \vdash\left\langle n_{i}^{\beta}\right\rangle \top$ for $n_{i} \in N-\bmod (\psi)$, then $\beta \leq \alpha_{i}$;

3. If $\psi \vdash\left\langle n_{i}^{\delta}\right\rangle \top$ for $n_{i} \in N-\bmod (\psi)$, then $\left\langle n_{i}^{\alpha_{i}}\right\rangle \top \vdash\left\langle n_{i}^{\delta}\right\rangle \top$;

4. If $\psi \vdash\left\langle n^{\gamma}\right\rangle \top$ for $n \notin N-\bmod (\psi)$, then $\left\langle n_{J}^{\alpha_{J}}\right\rangle \top \vdash\left\langle n^{\gamma}\right\rangle \top$.

With these results, we can now obtain uniqueness of our MNF's.

Theorem 3.3.6. Given $\psi_{1}, \psi_{2} \in M N F$, if $\psi_{1} \equiv \psi_{2}$ then $\psi_{1}=\psi_{2}$.

Proof. This now follows by an easy induction on the number of monomials in an MNF by the previous results.

This last theorem can be combined with Theorem 2.3.10 obtaining the next corollary.

Corollary 3.3.7. For every formula $\varphi$ there is a unique $\psi \in M N F$ such that $\varphi \equiv \psi$.

The uniqueness of MNF's will be key in proving completeness. It also has a couple of other nice corollaries.

Corollary 3.3.8. Each formula has a unique INF.

Corollary 3.3.9. Let $\varphi \in \mathbb{F}_{<n}$ for some $n$, and $\psi \in M N F$ such that $\varphi \equiv \psi$. Then $\psi \in \mathbb{F}_{<n}$.

Proof. As we have seen in Section 2.3, there is a procedure by which any formula $\varphi$ can be reduced to an equivalent $\psi_{\varphi} \in$ MNF that only contains modality bases occurring in $\varphi$, and so $\psi_{\varphi} \in \mathbb{F}_{<n}$. Moreover, by uniqueness, for every $\psi \in$ MNF such that $\varphi \equiv \psi$, we obtain that $\psi=\psi_{\varphi}$ an thus $\psi \in$ $\mathbb{F}_{<n}$.

In a next proposition we will prove that in a sense, initial truncations of MNF's are modally optimal approximations of the entire MNF. In order to precisely formulate what this means, we first need to recall that $\varphi \equiv_{n} \psi$ is defined as $\varphi \vdash \chi$ iff $\psi \vdash \chi$, for any $\chi \in \mathbb{F}_{<n+1}$.

We point out that the following proposition is a direct consequence of Lemma 3.2.14 through the arithmetical soundness and completeness of our calculus. However, since the current proof only hinges on purely modal facts together with 3.3.1 it is amenable to a setting where arithmetical completeness or soundness is not (yet) available. 
Proposition 3.3.10. Given $\psi:=\left\langle n_{0}^{\alpha_{0}}\right\rangle \top \wedge \ldots \wedge\left\langle n_{k}^{\alpha_{k}}\right\rangle \top \in M N F$ and $n \leq n_{k}$, provably in TSC:

$$
\left\langle n_{0}^{\alpha_{0}}\right\rangle \top \wedge \ldots \wedge\left\langle n_{k}^{\alpha_{k}}\right\rangle \top \equiv_{n} \bigwedge_{0 \leq i \leq J}\left\langle n_{i}^{\alpha_{i}}\right\rangle \top
$$

where $n_{J}=\mu(n, \psi)$.

Proof. This follows from Corollary 3.3.9 together with Items 3 and 4 of Corollary $3 \cdot 3 \cdot 5$.

\section{Arithmetical Completeness}

With the help of some of the results established in the previous subsection, we can state the following characterization of the derivability between formulas in MNF.

Proposition 3.3.11. For any $\psi_{0}, \psi_{1} \in M N F$, where $\psi_{0}:=\left\langle n_{0}^{\alpha_{0}}\right\rangle \top \wedge \ldots \wedge$ $\left\langle n_{k}^{\alpha_{k}}\right\rangle \top$ and $\psi_{1}:=\left\langle m_{0}^{\beta_{0}}\right\rangle \top \wedge \ldots \wedge\left\langle m_{j}^{\beta_{j}}\right\rangle \top, \psi_{0} \vdash \psi_{1}$ iff the following holds:

1. $m_{j} \leq n_{k} ;$

2. For any $m_{l} \in N-\bmod \left(\psi_{1}\right)$ and $n_{I}=\mu\left(m_{l}, \psi_{0}\right)$, we have $\beta_{l} \leq e^{n_{I}-m_{l}}\left(\alpha_{I}\right)$.

Proof. Assume $\psi_{0} \vdash \psi_{1}$. For a proof of Item 2, consider some $m_{l} \in \mathrm{N}-\bmod \left(\psi_{1}\right)$. Since $\psi_{0} \vdash \psi_{1}$ then, in particular, $\psi_{0} \vdash\left\langle m_{l}^{\beta_{l}}\right\rangle \top$ and so, by Corollary 3.3.5, Items 2 and 4, together with Proposition 3.3.2, we have that $\beta_{l} \leq e^{n_{I}-m_{l}}\left(\alpha_{I}\right)$. Moreover, since if $\psi_{0} \vdash \psi_{1}$ then $\psi_{0} \vdash\left\langle m_{j}^{\beta_{j}}\right\rangle \top$. Thus, Item 1 follows from Corollary $3 \cdot 3 \cdot 5$, Item 1.

For the other direction, assume Items 1 and 2 and consider any monomial $\left\langle m_{l}^{\beta_{l}}\right\rangle \top$ occurring in $\psi_{1}$. We can distinguish two cases: either $m_{l} \in$ $\mathrm{N}-\bmod \left(\psi_{0}\right)$ and then by Axiom $3 \psi_{0} \vdash\left\langle m_{l}^{\beta_{l}}\right\rangle \top$ or $m_{l} \notin \mathrm{N}-\bmod \left(\psi_{0}\right)$, but then by a combination of Axioms 5 and $3,\left\langle n_{I}^{\alpha_{I}}\right\rangle \top \vdash\left\langle m_{l}^{\beta_{l}}\right\rangle \top$, and so $\psi_{0} \vdash\left\langle m_{l}^{\beta_{l}}\right\rangle \top$. Therefore, $\psi_{0} \vdash \psi_{1}$.

Before proving the completeness of our system, we need to check the correctness of the derivable Rules (B1) and (B2) introduced in Proposition 3.3.4 of the previous subsection.

Lemma 3.3.12. Rules $\left(B_{1}\right)$ and $\left(B_{2}\right)$ are sound w.r.t. the FTP interpretation.

Proof. To check the soundness of $(\mathrm{BI})$, assume $\left(\mathrm{EA}^{+}\right)_{m}^{\beta} \subseteq \mathrm{Th}_{\psi}$. Since for $m>n_{k}, e^{m-n_{k}}(\beta) \in \operatorname{Lim}$, we can observe that $\left(\left(\mathrm{Th}_{\psi}\right)_{n_{k}}^{1}\right)_{n_{k}}^{e^{m-n_{k}}(\beta)} \equiv\left(\mathrm{Th}_{\psi}\right)_{n_{k}}^{e^{m-n_{k}}(\beta)}$, and then, by correctness of Axiom 5:

$$
\left(\mathrm{Th}_{\psi}\right)_{n_{k}}^{e^{m-n_{k}}(\beta)} \subseteq\left(\left(\mathrm{Th}_{\psi}\right)_{n_{k}}^{1}\right)_{m}^{\beta} \subseteq\left(\left(\mathrm{Th}_{\psi}\right)_{n_{k}}^{1}+\mathrm{EA}^{+}\right)_{m}^{\beta}
$$


Since $m>n_{k}$, we get that $\left(\left(\mathrm{Th}_{\psi}\right)_{n_{k}}^{1}+\mathrm{EA}^{+}\right)_{m}^{\beta} \subseteq\left(\mathrm{Th}_{\psi}\right)_{n_{k}}^{1}+\left(\mathrm{EA}^{+}\right)_{m}^{\beta}$ and by our assumption, $\left(\mathrm{Th}_{\psi}\right)_{n_{k}}^{1}+\left(\mathrm{EA}^{+}\right)_{m}^{\beta} \subseteq\left(\mathrm{Th}_{\psi}\right)_{n_{k}}^{1}$. Therefore, $\left(\mathrm{Th}_{\psi}\right)_{n_{k}}^{e^{m-n_{k}(\beta)}} \subseteq$ $\left(\mathrm{Th}_{\psi}\right)_{n_{k}}^{1}$.

With respect to (B2), we can observe that it holds trivially since the assumption of the antecedent together with Lemma 3.2.14 and Proposition 3.2.5, Item 3.2.5 yields $\mathrm{Th}_{\psi}$ is inconsistent.

With these two results, we are ready now to prove completeness of TSC:

Theorem 3.3.13. For any $\varphi, \psi \in \mathbb{F}_{\text {TSC }}$, if $\mathrm{EA}^{+} \vdash T h_{\psi} \subseteq T h_{\varphi}$, then the sequent $\varphi \vdash \psi$ is derivable in TSC.

Proof. W.1.o.g., let $\varphi, \psi \in$ MNF be such that $\varphi:=\left\langle n_{0}^{\alpha_{0}}\right\rangle \top \wedge \ldots \wedge\left\langle n_{k}^{\alpha_{k}}\right\rangle \top$ and $\psi:=\left\langle m_{0}^{\beta_{0}}\right\rangle \top \wedge \ldots \wedge\left\langle m_{j}^{\beta_{j}}\right\rangle \top$. By contraposition, assume $\varphi \not \forall \psi$. Hence by Proposition 3.3.11, either:

- $n_{k}<m_{j}$ or,

- There is $m_{l} \in \mathrm{N}-\bmod (\psi)$ and $n_{I}=\mu\left(m_{l}, \varphi\right)$, such that $\beta_{l}>e^{n_{I}-m_{l}}\left(\alpha_{I}\right)$.

Towards a contradiction assume that $\mathrm{EA}^{+} \vdash \mathrm{Th}_{\psi} \subseteq \mathrm{Th}_{\varphi}$. If $n_{k}<m_{j}$, notice that provably in $\mathrm{EA}^{+}$we have that $\left(\mathrm{EA}^{+}\right){ }_{m_{j}}^{\beta_{j}} \subseteq \mathrm{Th}_{\psi}$ and so, in particular we have that $\left(\mathrm{EA}^{+}\right)_{m_{j}}^{\beta_{j}} \subseteq \mathrm{Th}_{\varphi}$. Thus, applying (BI), together with Lemma 3.3.12 we obtain that

$$
\mathrm{EA}^{+} \vdash\left(\mathrm{Th}_{\varphi}\right)_{n_{k}}^{e^{m_{j}-n_{k}}(\beta)} \subseteq\left(\mathrm{Th}_{\varphi}+\operatorname{Con}_{n_{k}}\left(\mathrm{Th}_{\varphi}\right)\right)
$$

Therefore, $\mathrm{EA}^{+} \vdash \square_{\left(\mathrm{Th}_{\varphi}\right)_{n_{k}}^{1}} \operatorname{Con}_{n_{k}}\left(\left(\mathrm{Th}_{\varphi}\right)_{n_{k}}^{1}\right)$ and then $\mathrm{EA}^{+} \vdash \square_{\left(\mathrm{Th}_{\varphi}\right)_{n_{k}}^{1}} \perp$. Hence, $\mathbb{N}=\square\left(\mathrm{Th}_{\varphi}\right)_{n_{k}}^{1} \perp$ and so, $\left(\mathrm{Th}_{\varphi}\right)_{n_{k}}^{1}$ is inconsistent.

For the second case, we reason analogously but using (B2). 


\section{CHAPTER}

\section{Relational Semantics for TSC}

In this chapter we shall discuss relational semantics for TSC. We will start by defining a new model $\mathcal{J}$ which is proven to be universal for our logic. That is, any derivable sequent will hold -in a precise sense to be defined below- everywhere in the model whereas any non-derivable sequent will be refuted somewhere in the model. This frame $\mathcal{J}$ is a slight modification of Ignatiev's universal model $\mathcal{I}$.

The results of this chapter are included in [28].

\subsection{A Variation on Ignatiev's Frame}

In [30], K. Ignatiev introduced a frame which is a universal model for the variable-free fragment of GLP which has been intensively studied (see [14], [23], [29]).

This model will be based on special sequences of ordinals. In order to define them, we need the following central definition.

Definition 4.1.1. We define ordinal logarithm as $\ell(0):=0$ and $\ell\left(\alpha+\omega^{\beta}\right):=\beta$.

Thus, the set of worlds of this frame is defined as follows.

Definition 4.1.2. By $I g^{\omega}$ we denote the set of $\ell$-sequences or Ignatiev sequences. That is, the set of sequences $x:=\left\langle x_{0}, x_{1}, x_{2}, \ldots\right\rangle$ where for $i<\omega, x_{i+1} \leq \ell\left(x_{i}\right)$.

Given a $\ell$-sequence $x$, if all but finitely many of its elements are zero, we will write $\left\langle x_{0}, \ldots, x_{n}, \overrightarrow{0}\right\rangle$ to denote such sequence or even simply $\left\langle x_{0}, \ldots, x_{n}\right\rangle$ 
whenever $x_{n+1}=0$.

We can define relations $T_{n}$ in $\operatorname{Ig}^{\omega}$ by:

$$
x T_{n} y: \Leftrightarrow\left(\forall m<n x_{m}=y_{m} \wedge x_{n}>y_{n}\right) .
$$

The resulting structure $\mathcal{I}=\left\langle\operatorname{Ig}^{\omega},\left\{T_{n}\right\}_{n<\omega}\right\rangle$ is called the Ignatiev's frame.

To define $\mathcal{J}$ we will make use of the same set of $\ell$-sequences but we shall consider some minor modifications in the definition of the relations:

Definition 4.1.3. $\mathcal{J}_{\Lambda}:=\left\langle I,\left\{R_{n}\right\}_{n<\omega}\right\rangle$ is defined as follows:

$$
I:=\left\{x \in \lg ^{\omega}: x_{m}<\Lambda \text { for } m<\omega\right\}
$$

and

$$
x R_{n} y: \Leftrightarrow\left(\forall m \leq n x_{m}>y_{m} \wedge \forall m>n x_{m} \geq y_{m}\right) .
$$

Since $\Lambda$ was already fixed in the previous chapter, from now on we suppress the subindex $\Lambda$.

The observations collected in the next lemma all have elementary proofs. Basically, the lemma confirms that the $R_{n}$-relations are good to model provability logic and respect the increasing strength of the provability predicates $[n]$.

\section{Lemma 4.1.4.}

1. Each $R_{n}$ for $n<\omega$ is transitive: $x R_{n} y \wedge y R_{n} z \Rightarrow x R_{n} z$;

2. Each $R_{n}$ for $n<\omega$ is Noetherian: each non-empty $X \subseteq I$ has an $R_{n^{-}}$ maximal element $y \in X$, i.e., $\forall x \in X \neg y R_{n} x$;

3. The relations $R_{n}$ are monotone in $n$ in the sense that: $x R_{n} y \Rightarrow x R_{m} y$ whenever $n>m$.

Note that Item 2 is equivalent to stating that there are no infinite ascending $R_{n}$ chains. In other words, the converse of $R_{n}$ is well-founded.

We can also make the following observation.

Lemma 4.1.5. For any $x, y, z \in I$ and $n, m<\omega$ :

$$
x R_{n} y R_{m} z \Longrightarrow x R_{n} z \wedge x R_{m} z .
$$

Proof. Assume that $x R_{n} y$ and $y R_{m} z$. Then, we have that $x_{i}>y_{i} \geq z_{i}$ for all $i \leq n$ and for all $i>n$, we get that $x_{i} \geq y_{i} \geq z_{i}$. Hence, $x R_{n} z$. On the other hand, for any $i<\omega$, we have that $x_{i} \geq y_{i}$. Therefore, for all $i \leq m, x_{i}>z_{i}$ and for any $i>m, x_{i} \geq z_{i}$. Thus, $x R_{m} z$. 
We define the auxiliary relations $R_{n}^{\alpha}$ for any $n<\omega$ and $\alpha<\Lambda$. The idea is that the $R_{n}^{\alpha}$ relation will model the $\left\langle n^{\alpha}\right\rangle$ modality.

Definition 4.1.6. Given $x, y \in I$ and $R_{n}$ on $I$, we recursively define $x R_{n}^{\alpha} y$ as follows:

1. $x R_{n}^{0} y: \Leftrightarrow x=y$;

2. $x R_{n}^{1+\alpha} y: \Leftrightarrow \forall \beta<1+\alpha \exists z\left(x R_{n} z \wedge z R_{n}^{\beta} y\right)$.

In Definition 4.1.6, we could have given an equivalent formulation in terms of successor and limit ordinals as the next lemmata tell us.

Proposition 4.1.7. Given $x, y \in I, n<\omega$ and $\alpha<\Lambda$ :

$$
x R_{n}^{\alpha+1} y \Longleftrightarrow \exists z\left(x R_{n} z \wedge z R_{n}^{\alpha} y\right) .
$$

Proof. We make a case distinction on $\alpha$ with $\alpha=0$ being trivial. For $\alpha>0$, we have that $\alpha=1+\gamma$ for some $\gamma \leq \alpha$. With the help of this fact, we can reason as follows:

$$
\begin{aligned}
x R_{n}^{\alpha+1} y & \Leftrightarrow x R_{n}^{1+\gamma+1} y ; \\
& \Leftrightarrow \forall \beta<1+\delta+1 \exists z\left(x R_{n} z \wedge z R_{n}^{\beta} y\right) ; \\
& \Rightarrow \exists z\left(x R_{n} z \wedge z R_{n}^{\alpha} y\right), \text { in particular. }
\end{aligned}
$$

Thus, $x R_{n}^{\alpha+1} y \Rightarrow \exists z\left(x R_{n} z \wedge z R_{n}^{\alpha} y\right)$. For the right-to-left implication we proceed analogously:

$$
\begin{aligned}
\exists z\left(x R_{n} z \wedge z R_{n}^{\alpha} y\right) \Leftrightarrow & \exists z\left(x R_{n} z \wedge z R_{n}^{1+\gamma} y\right) ; \\
\Leftrightarrow & \exists z\left(\left(x R_{n} z \wedge z R_{n}^{1+\gamma} y\right) \wedge\right. \\
& \left.\forall \beta<1+\gamma \exists z^{\prime}\left(z R_{n} z^{\prime} \wedge z^{\prime} R_{n}^{\beta} y\right)\right) ; \\
\Rightarrow & \forall \beta^{\prime}<1+\gamma+1 \exists u\left(x R_{n} u \wedge u R_{n}^{\beta^{\prime}} y\right) ; \\
\Rightarrow & x R_{n}^{1+\gamma+1} y ; \\
\Rightarrow & x R_{n}^{\alpha+1} y .
\end{aligned}
$$

We can also make a similar simple observation for limit ordinals.

Proposition 4.1.8. Let $x, y \in I, n<\omega$ and $\lambda<\Lambda$ such that $\lambda \in \operatorname{Lim}$ :

$$
x R_{n}^{\lambda} y \Longleftrightarrow \forall \beta<\lambda x R_{n}^{1+\beta} y \Longleftrightarrow \forall \beta, 0<\beta<\lambda, x R_{n}^{\beta} y .
$$


Proof. Since the last equivalence is straightforward, we just show that:

$$
x R_{n}^{\lambda} y \Longleftrightarrow \forall \beta<\lambda x R_{n}^{1+\beta} y .
$$

For left-to-right implication, notice that if $x R_{n}^{\lambda} y$ then by Definition 4.1.6, we have that

$$
\forall \beta<\lambda \exists u\left(x R_{n} u \wedge u R_{n}^{\beta} y\right) .
$$

Therefore, since $\lambda \in$ Lim we obtain that $\forall \beta<\lambda \exists u\left(x R_{n} u \wedge u R_{n}^{1+\beta} y\right)$ and so, by Definition 4.1.6, we have that

$$
\forall \beta<\lambda \forall \delta<1+\beta \exists u, v\left(x R_{n} u \wedge u R_{n} v \wedge v R_{n}^{\delta} y\right) .
$$

By transitivity of $R_{n}$, we get that $\forall \beta<\lambda \quad x R_{n}^{1+\beta} y$. For the other direction, if $\forall \beta<\lambda x R_{n}^{1+\beta} y$, then in particular, $\forall \beta<\lambda x R_{n}^{\beta+1} y$ and then, by Proposition 4.1.7, $\forall \beta<\lambda \exists u\left(x R_{n} u \wedge u R_{n}^{\beta} y\right)$, that is, $x R_{n}^{\lambda} y$.

It is easy to see that for example $\langle\omega, \overrightarrow{0}\rangle R_{0}^{1+n}\langle m, \overrightarrow{0}\rangle$ for each $n, m \in \omega$, so that also $\langle\omega, \overrightarrow{0}\rangle R_{0}^{\omega}\langle m, \overrightarrow{0}\rangle$ for each $m \in \omega$. Clearly, we do not have $\langle\omega, \overrightarrow{0}\rangle R_{0}^{\omega+1}\langle m, \overrightarrow{0}\rangle$ for any $m<\omega$ but we do have $\langle\omega+1, \overrightarrow{0}\rangle R_{0}^{\omega+1}\langle m, \overrightarrow{0}\rangle$ for all $m<\omega$.

We also note that the dual definition $x \bar{R}_{n}^{0} y: \Leftrightarrow x=y$; and $x \bar{R}_{n}^{1+\alpha} y: \Leftrightarrow$ $\forall \beta<1+\alpha \exists z\left(x \bar{R}_{n}^{\beta} z \wedge z \bar{R}_{n} y\right)$ does not make much sense on our frames. For example we could have $\langle\omega, \overrightarrow{0}\rangle \bar{R}_{0}^{\alpha}\langle 0, \overrightarrow{0}\rangle$ for any ordinal $\alpha>0$.

With the the auxiliary relations $R_{n}^{\alpha}$, we give the following definition for a formula $\varphi$ being true in a point $x$ of $\mathcal{J}$.

Definition 4.1.9. Let $x \in I$ and $\varphi \in \mathbb{F}_{T S C}$. By $x \Vdash \varphi$ we denote the validity of $\varphi$ in $x$ that is recursively defined as follows:

- $x \Vdash \top$ for all $x \in I$;

- $x \Vdash \varphi \wedge \psi$ iff $x \Vdash \varphi$ and $x \Vdash \psi$;

- $x \Vdash\left\langle n^{\alpha}\right\rangle \varphi$ iff there is $y \in I, x R_{n}^{\alpha} y$ and $y \Vdash \varphi$.

In the following lemma we present some easy observations on the $R_{n}^{\alpha}$ relations which among others tell us that all the $R_{n}^{1+\alpha}$ serve the purpose of a provability predicate for any $n<\omega$ and $\alpha<\Lambda$.

\section{Lemma 4.1.10.}

1. Each $R_{n}^{1+\alpha}$ for $n<\omega$ and $\alpha$ an ordinal is transitive: $x R_{n}^{1+\alpha} y \wedge y R_{n}^{1+\alpha} z \Rightarrow$ $x R_{n}^{1+\alpha} z$;

2. Each $R_{n}^{1+\alpha}$ for $n<\omega$ and $\alpha$ an ordinal is Noetherian: each non-empty $X \subseteq I$ has an $R_{n}^{1+\alpha}$-maximal element $y \in X$, i.e., $\forall x \in X \neg y R_{n}^{1+\alpha} x$; 

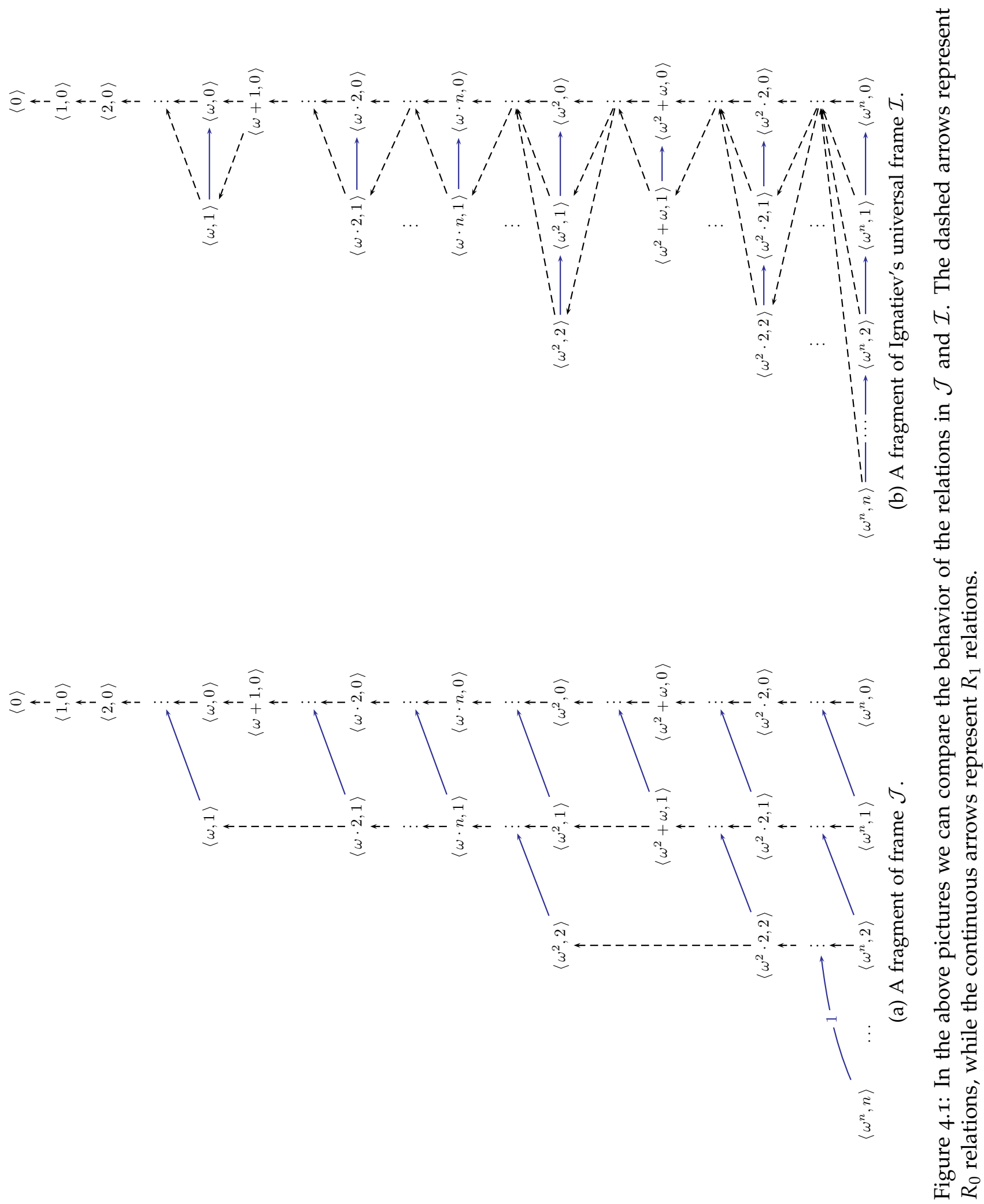
3. The relations $R_{n}^{1+\alpha}$ are monotone in $n$ in the sense that: $x R_{n}^{1+\alpha} y \Rightarrow x R_{m}^{1+\alpha} y$ whenever $n>m$;

4. The relations $R_{n}^{1+\alpha}$ are monotone in $1+\alpha$ in the sense that: $x R_{n}^{1+\alpha} y \Rightarrow$ $x R_{n}^{1+\beta} y$ whenever $1+\beta<1+\alpha$.

Proof. The first three items follow directly from Lemma 4.1.4 by an easy transfinite induction. The last item is also easy. lations.

The following lemma states the polytransitivity condition for the $R_{n}^{\alpha}$ re-

Lemma 4.1.11 (Polytransitivity). For any $x, y, z \in I, n, m<\omega$ and $\alpha, \beta<\Lambda$ with $0<\alpha, \beta$ :

$$
x R_{n}^{\alpha} y R_{m}^{\beta} z \Longrightarrow x R_{n}^{\alpha} z \wedge x R_{m}^{\beta} z .
$$

Proof. We assume $x R_{n}^{\alpha} y$ and $y R_{m}^{\beta} z$ and set out to prove $x R_{n}^{\alpha} z$. We proceed by induction on $\alpha$. For the base case, let $\alpha=1$. We can observe that by Lemma 4.1.10, Item 4, since $y R_{m}^{\beta} z$, then $y R_{m} z$. Therefore, the implication follows from Lemma 4.1.5.

For $\alpha \in$ Succ, let $\alpha:=\alpha^{\prime}+1$. By Proposition 4.1.7, if $x R_{n}^{\alpha} y$, then $\exists u \in I$ such that $x R_{n} u$ and $u R_{n}^{\alpha^{\prime}} y$. Since $u R_{n}^{\alpha^{\prime}} y$ and $y R_{m}^{\beta} z$, by the I.H. we have that $u R_{n}^{\alpha^{\prime}} z$ and thus, $x R_{n}^{\alpha} z$.

For $\alpha \in$ Lim, by Proposition 4.1.8, we have that for all $\delta<\alpha, x R_{n}^{1+\delta} y$ and $y R_{m}^{\beta} z$. Thus, by the I.H. we get that $x R_{n}^{1+\delta} z$ for all $\delta<\alpha$. Hence, $x R_{n}^{\alpha} z$.

To prove that $x R_{m}^{\beta} z$ we proceed by induction on $\beta$. The base and limit cases are analogous to the ones of the induction on $\alpha$. For the case $\beta \in$ Succ, let $\beta:=\beta^{\prime}+1$. With the help of Proposition 4.I.7 we have that $\exists u \in I$ such that $y R_{m} u$ and $u R_{m}^{\beta^{\prime}} z$. By Lemma 4.1.10, Item 4, since $x R_{n}^{\alpha} y$, then $x R_{n} y$ and by Lemma 4.1.5 $x R_{m} u$. Therefore, $x R_{m}^{\beta} y$.

\section{A characterization for transfinite accessibility}

The intuitive idea behind the $x R_{n}^{\alpha} y$ assertion, is that this tells us that there exists a chain of 'length' $\alpha$ of $R_{n}$ steps leading from the point $x$ up to the point $y$. The following useful lemma tries to capture this intuition.

Lemma 4.1.12. For $x, y \in I, \alpha<\Lambda$ and $n<\omega$ we have that the following are equivalent

1. $x R_{n}^{1+\alpha} y$

2. There exists a collection $c^{1+\alpha}:=\left\{x_{\delta}^{1+\alpha}\right\}_{\delta<1+\alpha}$ so that

a) $x R_{n} x_{\delta}^{1+\alpha}$ for any $x_{\delta}^{1+\alpha} \in c^{1+\alpha}$, 
b) $x_{0}^{1+\alpha}=y$ and,

c) for any $\delta^{\prime}<\delta<1+\alpha$ we have $x_{\delta}^{1+\alpha} R_{n} x_{\delta^{\prime}}^{1+\alpha}$.

Proof. By induction on $\alpha$. The base case is immediate if we consider the collection $\{y\}$. For $\alpha \in$ Succ, let $\alpha:=\alpha^{\prime}+1$. By Proposition 4.1.7, we have that $x R_{n}^{1+\alpha^{\prime}+1} y$ iff there is $z$ such that $x R_{n} z$ and $z R_{n}^{1+\alpha^{\prime}} y$. Thus, by the I.H. $x R_{n}^{1+\alpha^{\prime}+1} y$ iff there is $z$ such that $x R_{n} z$ and there is a collection $c^{1+\alpha^{\prime}}:=\left\{z_{\delta}^{1+\alpha^{\prime}}\right\}_{\delta<1+\alpha^{\prime}}$ such that:

1. $z R_{n} z_{\delta}^{1+\alpha^{\prime}}$ for any $z_{\delta}^{1+\alpha^{\prime}} \in c^{1+\alpha^{\prime}}$,

2. $z_{0}^{1+\alpha^{\prime}}=y$ and,

3. for any $\delta^{\prime}<\delta<1+\alpha^{\prime}$ we have $z_{\delta}^{1+\alpha^{\prime}} R_{n} z_{\delta^{\prime}}^{1+\alpha^{\prime}}$.

Thus, we just need to consider the collection $c^{1+\alpha^{\prime}+1}$ defined as follows:

$$
x_{\delta}^{1+\alpha^{\prime}+1}= \begin{cases}z & \text { if } \delta=1+\alpha^{\prime} \\ z_{\delta}^{1+\alpha^{\prime}} & \text { otherwise }\end{cases}
$$

To check the other implication, assume we have a collection $c^{1+\alpha^{\prime}+1}$ satisfying the properties $2 a, 2 b$ and $2 c$. Then, we have that $x R_{n} x_{1+\alpha^{\prime}}^{1+\alpha^{\prime}+1}$ and applying the I.H. to the collection $\left\{x_{\delta}^{1+\alpha^{\prime}+1}\right\}_{\delta<1+\alpha^{\prime}}$, we get that $x_{1+\alpha^{\prime}}^{1+\alpha^{\prime}+1} R_{n}^{1+\alpha^{\prime}} y$. Hence, $x R_{n}^{1+\alpha^{\prime}+1} y$.

For $\alpha \in \operatorname{Lim}$, let us first prove the implication from Item 1 to Item 2. By Proposition 4.1.8 we have that $x R_{n}^{\alpha} y$ iff for any $\gamma<\alpha, x R_{n}^{1+\gamma} y$. Thus, for each $\gamma<\alpha$, we have a collection $c^{1+\gamma}:=\left\{z_{\delta}^{1+\gamma}\right\}_{\delta<1+\gamma}$ satisfying the properties $2 a$, $2 b$ and $2 c$. In order to build the collection $c^{\alpha}:=\left\{x_{\gamma}^{\alpha}\right\}_{\gamma<\alpha}$, we set $x_{0}^{\alpha}$ to be $y$ and define $x_{1+\gamma}^{\alpha}$ uniformly as follows:

$$
\left(x_{1+\gamma}^{\alpha}\right)_{m}:= \begin{cases}y_{m} & \text { in case } m>n, \\ \min \left\{\left(z_{1+\gamma}^{\zeta}\right)_{m}: z_{1+\gamma}^{\zeta} \in c^{\zeta} \text { for } \zeta>1+\gamma\right\} & \text { in case } m=n, \\ e^{n-m}\left(\left(x_{1+\gamma}^{\alpha}\right)_{n}\right) & \text { in case } m<n .\end{cases}
$$

The reason why we consider the minimum value for $\left(x_{1+\gamma}^{\alpha}\right)_{n}$ is that minimum means highest in the $R_{n}$ relation. Therefore, the minimum value guarantees that we are sufficiently down to reach $y$ but, at the same time, that we still have room enough to build the remaining members of our collection.

First, we check that $c^{\alpha} \subseteq I$. Since $x_{0}^{\alpha}=y \in I$, we only check $x_{\gamma}^{\alpha} \in I$ for $\gamma>0$. Since for $m>n,\left(x_{\gamma}^{\alpha}\right)_{m}=y_{m}$ and $y \in I$, it suffices to establish the following: 
- $\ell\left(\left(x_{\gamma}^{\alpha}\right)_{n}\right) \geq\left(x_{\gamma}^{\alpha}\right)_{n+\mathbf{1}}$. By definition, there is $\zeta^{*}>\gamma$, such that $\left(x_{\gamma}^{\alpha}\right)_{n}=$ $\left(z_{\gamma}^{\zeta^{*}}\right)_{n}$ and $z_{\gamma}^{\zeta^{*}} \in I$. Hence $\ell\left(\left(z_{\gamma}^{\zeta^{*}}\right)_{n}\right) \geq\left(z_{\gamma}^{\zeta^{*}}\right)_{n+1}$. By the I.H. Items $2 b$ and $2 c z_{\gamma}^{\zeta^{*}} R_{n} y$, and so $\left(z_{\gamma}^{\zeta^{*}}\right)_{n+1} \geq y_{n+1}=\left(x_{\gamma}^{\alpha}\right)_{n+1}$. Thus, $\ell\left(\left(x_{\gamma}^{\alpha}\right)_{n}\right) \geq\left(x_{\gamma}^{\alpha}\right)_{n+1}$.

- $\ell\left(\left(x_{\gamma}^{\alpha}\right)_{m}\right) \geq\left(x_{\gamma}^{\alpha}\right)_{m+1}$ for $m<n$. By definition we have that $\left(x_{\gamma}^{\alpha}\right)_{m+1}=$ $e^{n-(m+1)}\left(\left(x_{\gamma}^{\alpha}\right)_{n}\right)$ and $\left(x_{\gamma}^{\alpha}\right)_{m}=e^{n-m}\left(\left(x_{\gamma}^{\alpha}\right)_{n}\right)$. Thus,

$$
\ell\left(\left(x_{\gamma}^{\alpha}\right)_{m}\right)=\ell\left(e^{n-m}\left(\left(x_{\gamma}^{\alpha}\right)_{n}\right)\right)=e^{n-(m+1)}\left(\left(x_{\gamma}^{\alpha}\right)_{n}\right)=\left(x_{\gamma}^{\alpha}\right)_{m+1} .
$$

With respect to the conditions $2 a, 2 b$ and $2 c$, Item $2 b$ follows by definition. Regarding Item $2 a$, pick an arbitrary $x_{\delta}^{\alpha} \in c^{\alpha}$. By the I.H. we know that for any point $z_{\delta}^{1+\gamma} \in c^{1+\gamma}$ for $\gamma<\alpha$ we have that $x R_{n} z_{\delta}^{1+\gamma}$. Then:

- $x R_{n} z_{0}^{1+\gamma}$, that is, $x R_{n} y$ and so, $x_{m} \geq y_{m}=\left(x_{\delta}^{\alpha}\right)_{m}$ for $m>n$;

- $x_{n}>\min \left\{\left(z_{\delta}^{\zeta}\right)_{n}: z_{\delta}^{\zeta} \in c^{\zeta}\right.$ for $\left.\zeta>\delta\right\}=\left(x_{\delta}^{\alpha}\right)_{n}$;

- For $m<n$ and $\zeta>\delta$, we have that $x_{m}>\left(z_{\delta}^{\zeta}\right)_{m} \geq e^{n-m}\left(\left(z_{\delta}^{\zeta}\right)_{n}\right)$. Therefore, $x_{m}>e^{n-m}\left(\left(x_{\delta}^{\alpha}\right)_{n}\right)$.

Hence, $x R_{n} x_{\delta}^{\alpha}$ for any $\delta<\alpha$. Concerning Item $2 c$, by definition of $c^{\alpha}$, to obtain that for any $\delta^{\prime}<\delta<\alpha$, we have that $x_{\delta}^{\alpha} R_{n} x_{\delta^{\prime}}^{\alpha}$ it suffices to check that $\left(x_{\delta^{\prime}}^{\alpha}\right)_{n}<\left(x_{\delta}^{\alpha}\right)_{n}$ considering that $\left(x_{\delta^{\prime}}^{\alpha}\right)_{m}=\left(x_{\delta}^{\alpha}\right)_{m}$ for $m>n$ and $\left(x_{\delta^{\prime}}^{\alpha}\right)_{m}<\left(x_{\delta}^{\alpha}\right)_{m}$ for $m \leq n$. We assume $\delta^{\prime} \neq 0$ since that case is easy.

We can observe that there are $\zeta^{*}$ and $\xi^{*}$ such that $\alpha>\zeta^{*}>\delta$ and $\alpha>\xi^{*}<\delta^{\prime}$, satisfying the following:

$$
\left(x_{\delta}^{\alpha}\right)_{n}=\left(z_{\delta}^{\zeta^{*}}\right)_{n} \text { and }\left(x_{\delta^{\prime}}^{\alpha}\right)_{n}=\left(z_{\delta^{\prime}}^{\xi^{*}}\right)_{n} .
$$

Since $\delta>\delta^{\prime}$, there is $z_{\delta^{\prime}}^{\zeta^{*}} \in c^{\zeta^{*}}$ and by the I.H. $z_{\delta}^{\zeta^{*}} R_{n} z_{\delta^{\prime}}^{\zeta^{*}}$. Thus, $\left(z_{\delta^{\prime}}^{\zeta^{*}}\right)_{n}<\left(z_{\delta}^{\zeta^{*}}\right)_{n}$. Since $\left(x_{\delta^{\prime}}^{\alpha}\right)_{n}$ is obtained by considering the minimum, we have have that

$$
\left(x_{\delta^{\prime}}^{\alpha}\right)_{n}=\left(z_{\delta^{\prime}}^{\tau^{*}}\right)_{n} \leq\left(z_{\delta^{\prime}}^{\zeta^{*}}\right)_{n}<\left(z_{\delta}^{\zeta^{*}}\right)_{n}=\left(x_{\delta}^{\alpha}\right)_{n}
$$

That is, $\left(x_{\delta^{\prime}}^{\alpha}\right)_{n}<\left(x_{\delta}^{\alpha}\right)_{n}$.

We know prove the implication from Item 2 to Item 1 . Thus, we assume there is a collection $c^{\alpha}$ satisfying conditions $2 a, 2 b$ and $2 c$. We will show that $x R_{n}^{\alpha} y$. First we prove the following by induction on $\gamma$ :

$$
\text { For any } x_{\gamma}^{\alpha} \in c^{\alpha}, x_{\gamma}^{\alpha} R_{n}^{\gamma} y \text {. }
$$

The base case is immediate since we have that $x_{0}^{\alpha}=y$ and $y R_{n}^{0} y$. For the successor step, let $\gamma=\gamma^{\prime}+1$. Since by assumption $x_{\gamma}^{\alpha} R_{n} x_{\gamma^{\prime}}^{\alpha}$ and by the I.H. 
$x_{\gamma^{\prime}}^{\alpha} R_{n}^{\gamma^{\prime}} y$, then $x_{\gamma}^{\alpha} R_{n}^{\gamma^{\prime}+1} y$. For the limit case, assume as the I.H. that for any $\delta<\gamma, x_{\delta}^{\alpha} R_{n}^{\delta} y$. By assumption we have that $x_{\gamma}^{\alpha} R_{n} x_{\delta}^{\alpha}$ for any $\delta<\gamma$. Thus, $x_{\gamma}^{\alpha} R_{n} x_{\delta}^{\alpha}$ and $x_{\delta}^{\alpha} R_{n}^{\delta} y$ for any $\delta<\gamma$, and so, $x_{\gamma}^{\alpha} R_{n}^{\gamma} y$.

Hence, for any $\gamma<\alpha$ we have that $x R_{n} x_{\gamma}^{\alpha}$ and $x_{\gamma}^{\alpha} R_{n}^{\gamma} y$, that is, $x R_{n}^{\alpha} y$.

We shall now provide another characterization of the $R_{n}^{1+\alpha}$ relations that is easier to work with. To this end, let us for convenience define

$$
x R_{-1}^{\zeta} y \quad: \Leftrightarrow \forall n>0 \quad x_{n} \geq y_{n} .
$$

With this notation the following theorem makes sense.

Theorem 4.1.13 (Characterization Theorem). For $x, y \in I$ and $n$ with $0 \leq n<\omega$ we have that the following are equivalent

1. $x R_{n}^{1+\alpha} y$;

2. $x_{n} \geq y_{n}+\left(1+e\left(y_{n+1}\right)\right) \cdot(1+\alpha)$ and $x R_{n-1}^{e(1+\alpha)} y$;

3.

$$
\begin{array}{ll}
x_{n} \geq y_{n}+\left(1+e\left(y_{n+1}\right)\right) \cdot(1+\alpha) & \text { and, } \\
x_{m}>y_{m} \text { for } m<n & \text { and, } \\
x_{m} \geq y_{m} \text { for } m>n . &
\end{array}
$$

We dedicate the remainder of this subsection to prove this theorem and move there through a series of lemmas. The first lemma in this series is pretty obvious. It tells us that if we can move from $x$ to $y$ in $\alpha$ many steps, then the distance between $x_{n}$ and $y_{n}$ must allow $\alpha$ many steps; That is, they lie at least $\alpha$ apart.

Lemma 4.1.14. For $x, y \in I$ and $n<\omega$ and any ordinal $\alpha<\Lambda$, if $x R_{n}^{\alpha} y$, then $x_{n} \geq y_{n}+\alpha$.

Proof. By an easy induction on $\alpha$. The base case is immediate since $x=y$. For $\alpha \in$ Succ, we just need to apply Proposition 4.1.7 and the I.H. The case $\alpha \in$ Lim follows from Proposition 4.1.8 and the I.H.

However, how many $R_{n}$ steps one can make is not entirely determined by the $n$ coordinates of the points. For example, there is just a single $R_{0}$ step from the point $\langle\omega \cdot 2,1\rangle$ to the point $\langle\omega, 1\rangle$ whereas these points lie $\omega$ apart on the ' 0 coordinate'. The following lemma tells us how for $R_{n}$ steps, the $n$-th coordinates are affected by the values of the $n+1$-th coordinate.

Lemma 4.1.15. For $x, y \in I$ and $n<\omega$ with $x R_{n}^{1+\alpha} y$, we have

$$
x_{n} \geq y_{n}+e\left(y_{n+1}\right) \cdot(1+\alpha) \text {. }
$$


In order to give a smooth presentation of this proof, we first give two simple technical lemmas with useful observations on the ordinals and ordinal functions involved.

Lemma 4.1.16. For $\alpha, \beta$ and $\gamma$ ordinals we have

1. $\ell(\beta) \geq 1+\alpha \Longleftrightarrow \beta \in e(1+\alpha) \cdot(1+$ Ord $)$;

2. If $(1+\alpha)<\beta$ and $\gamma \in e(\beta) \cdot(1+$ Ord $)$, then $\gamma \in e(1+\alpha) \cdot(1+$ Ord $)$;

3. $e(\beta+(1+\alpha))=e(\beta) \cdot e(1+\alpha)$;

Proof. The first two items can easily be seen by using a Cantor normal form expression with base $\omega$. For Item 1 , we use the fact that $\beta \in$ Lim together with that if $\ell(\beta) \geq 1+\alpha$, then $\beta \geq e(\ell(\beta)) \geq e(1+\alpha)$. For Items 2 and 3 we use that $e(1+\omega)=\omega^{1+\omega}=\omega^{1} \cdot \omega^{\omega}$.

Lemma 4.1.17. For $x, y \in I$ and $n<\omega$, we have that $x R_{n} y \Longrightarrow x_{n} \geq y_{n}+$ $e\left(x_{n+1}\right)$.

Proof. For $y_{n}=0$ the lemma is straightforward. Hence, we assume $y_{n}>0$ and proceed by a case distinction on $x_{n}$. We can observe that since $x R_{n} y$, then $x_{n}>y_{n}$ and thus, $x_{n} \neq 0$. If $x_{n} \in$ Succ then the lemma is trivial since $e\left(x_{n+1}\right)=0$. If $x_{n}$ is an additively indecomposable limit ordinal, then for any $\alpha, \beta<x_{n}$, we have that $x_{n}>\alpha+\beta$. Thus, since $x_{n}>y_{n}$ and $x_{n} \geq e\left(x_{n+1}\right)$, we can easily check that $x_{n} \geq y_{n}+e\left(x_{n+1}\right)$.

Otherwise, we can rewrite $x_{n}$ as $\alpha+e(\beta)$ for some $\beta \geq x_{n+1}$, and $y_{n}$ as $\delta+\omega^{\gamma}$. If $y_{n} \leq \alpha$ then clearly $x_{n} \geq y_{n}+e\left(x_{n+1}\right)$. For the remaining case, if $\alpha=\delta$ and $\gamma<\beta$, then we have that $\omega^{\gamma}+e(\beta)=e(\beta)$ and thus, $\alpha+e(\beta)=$ $\delta+\omega^{\gamma}+e(\beta) \geq y_{n}+e\left(x_{n+1}\right)$.

With these technical lemmas at hand we can now prove Lemma 4.1.15.

Proof. By induction on $\alpha$. For $\alpha:=\mathbf{0}$, we check that $x_{n} \geq y_{n}+e\left(y_{n+1}\right)$. Note that since $x R_{n} y$, then $x_{n} \geq y_{n}+e\left(x_{n+1}\right)$ and $x_{n+1} \geq y_{n+1}$, whence $x_{n} \geq y_{n}+$ $e\left(y_{n+1}\right)$.

For $\alpha:=\beta+1$, if $x R_{n}^{1+\beta+1} y$ then there is $z \in I$ such that $x R_{n} z$ and $z R_{n}^{1+\beta} y$. Thus, by the I.H. we have the following:

1. $x_{n} \geq z_{n}+e\left(z_{n+1}\right)$;

2. $z_{n} \geq y_{n}+e\left(y_{n+1}\right) \cdot(1+\beta)$.

Therefore, $x_{n} \geq y_{n}+e\left(y_{n+1}\right) \cdot(1+\beta)+e\left(z_{n+1}\right)$. Since $e\left(z_{n+1}\right) \geq e\left(y_{n+1}\right)$ then $x_{n} \geq y_{n}+e\left(y_{n+1}\right) \cdot(1+\beta)+e\left(y_{n+1}\right)$, i.e., $x_{n} \geq y_{n}+e\left(y_{n+1}\right) \cdot(1+\beta+1)$.

For $\alpha \in$ Lim, we notice that by the I.H. we have that $x_{n} \geq y_{n}+e\left(y_{n+1}\right)$. $(1+\delta)$ for any $\delta<\alpha$. Thus, $x_{n} \geq y_{n}+e\left(y_{n+1}\right) \cdot(1+\alpha)$. 
Combining Lemma 4.1.15 and Lemma 4.1.14 we get the following.

Corollary 4.1.18. For $x, y \in I$ and $n<\omega$ we have that

$$
x R_{n}^{1+\alpha} y \Longrightarrow x_{n} \geq y_{n}+\left(1+e\left(y_{n+1}\right)\right) \cdot(1+\alpha) .
$$

Proof. For $y_{n+1}>0$, we have that $e\left(y_{n+1}\right) \in \operatorname{Lim}$ and thus, $1+e\left(y_{n+1}\right)=$ $e\left(y_{n+1}\right)$. Therefore, it follows immediately from Lemma 4.1.15. If $y_{n+1}=$ 0 , then $y_{n}+\left(1+e\left(y_{n+1}\right)\right) \cdot(1+\alpha)=y_{n}+(1+\alpha)$ and so, it follows from Lemma 4.1.14.

This corollary takes care of part of the implication from Item (1) to Item (2) in the characterization theorem (Theorem 4.1.13). We will now focus on the implication from Item (3) to Item (1) but before we do so, we first formulate a simple yet useful lemma.

Lemma 4.1.19. For $x, y \in I$ and $m>0$, if $x R_{n+m} y$, then $x_{n} \geq y_{n}+e\left(x_{n+1}\right)$.

Proof. Since $R_{n+m}$ is contained in $R_{n}$, if $x R_{n+m} y$ then $x R_{n} y$ and thus by Lemma 4.1.17, $x_{n} \geq y_{n}+e\left(x_{n+1}\right)$.

With this technical lemma we can obtain the next step in the direction from Item (3) to Item (1) in the characterization theorem (Theorem 4.1.13).

Lemma 4.1.20. For $x, y \in I$ and $n<\omega$ we have that if

$$
\begin{array}{ll}
x_{n} \geq y_{n}+\left(1+e\left(y_{n+1}\right)\right) \cdot(1+\alpha) & \text { and, } \\
x_{m}>y_{m} \text { for } m<n & \text { and, } \\
x_{m} \geq y_{m} \text { for } m>n, &
\end{array}
$$

then

$$
x R_{n}^{1+\alpha} y
$$

Proof. First, we assume

1. $x_{n} \geq y_{n}+\left(1+e\left(y_{n+1}\right)\right) \cdot(1+\alpha)$;

2. $x_{m}>y_{m}$ for $m<n$;

3. $x_{m} \geq y_{m}$ for $m>n$.

We use Lemma 4.1.12 whence are done if we can find a collection $c^{1+\alpha}=$ $\left\{z_{\delta}^{1+\alpha}\right\}_{\delta<1+\alpha}$ so that

1. $x R_{n} z_{\delta}^{1+\alpha}$ for any $z_{\delta}^{1+\alpha} \in c^{1+\alpha}$,

2. $z_{0}^{1+\alpha}=y$ and,

3. for any $\delta^{\prime}<\delta<1+\alpha$ we have $z_{\delta}^{1+\alpha} R_{n} z_{\delta^{\prime}}^{1+\alpha}$. 
We set $z_{0}^{1+\alpha}:=y$ and for $\gamma>0$, we define $z_{\gamma}^{1+\alpha}$ uniformly as follows:

$$
\left(z_{\gamma}^{1+\alpha}\right)_{m}:= \begin{cases}y_{m} & \text { in case } m>n \\ y_{n}+\left(1+e\left(y_{n+1}\right)\right) \cdot \gamma & \text { in case } m=n \\ y_{m}+e\left(\left(z_{\gamma}^{1+\alpha}\right)_{m+1}\right) & \text { in case } m<n .\end{cases}
$$

We make a collection of simple observations:

I For any $\gamma<1+\alpha$ and $m<\omega$, we have that $\left(z_{\gamma}^{1+\alpha}\right)_{m+1} \leq \ell\left(\left(z_{\gamma}^{1+\alpha}\right)_{m}\right)$ and so, each $z_{\gamma}^{1+\alpha}$ is an element of $I$;

II We now see that $x R_{n} z_{\gamma}^{1+\alpha}$ for each $\gamma<\alpha$. For $m>n$ we obviously have that $x_{m} \geq\left(z_{\gamma}^{1+\alpha}\right)_{m}$ and also $x_{n}>\left(z_{\gamma}^{1+\alpha}\right)_{n}$ is clear. For $m<n$, first we can observe that from assumptions 1,2 and 3 we get that $x R_{n} y$. Using this fact, we check by induction on $n-m$ that $x_{m}>\left(z_{\gamma}^{1+\alpha}\right)_{m}$. For the base case, since $x R_{n} y$, using Lemma 4.1.19 we have that

$$
\begin{aligned}
x_{n-1} & \geq y_{n-1}+e\left(x_{n}\right) \\
& \geq y_{n-1}+e\left(y_{n}+\left(1+e\left(y_{n+1}\right)\right) \cdot(1+\alpha)\right) \\
& >y_{n-1}+e\left(\left(z_{\gamma}^{1+\alpha}\right)_{n}\right) .
\end{aligned}
$$

For the inductive step, assume $x_{n-m}>\left(z_{\gamma}^{1+\alpha}\right)_{n-m}$ as the I.H. Hence, by Lemma 4.1.19 $x_{n-(m+1)} \geq y_{n-(m+1)}+e\left(x_{n-m}\right)$. Combining this with the I.H. we get that $x_{n-(m+1)}>\left(z_{\gamma}^{1+\alpha}\right)_{n-(m+1)}$;

III $x_{0}=y$ by definition;

IV By strict monotonicity of $e$, we see that for any $\gamma^{\prime}<\gamma<\alpha$ we have $z_{\gamma}^{1+\alpha} R_{n} z_{\gamma^{\prime}}^{1+\alpha}$

We are now ready to combine all the above observations and obtain a proof for the characterization theorem (Theorem 4.1.13).

Proof. From Item 2 to Item 3 is easy and from Item 3 to Item 1 is Lemma 4.1.20 so we focus on the remaining implication, that is:

$$
x R_{n}^{1+\alpha} y \Longrightarrow x_{n} \geq y_{n}+\left(1+e\left(y_{n+1}\right)\right) \cdot(1+\alpha) \text { and } x R_{n-1}^{e(1+\alpha)} y
$$

for any $x, y \in I$ and $n$ with $0 \leq n<\omega$.

As mentioned before, half of this implication follows from Corollary 4.1.18 so that it remains to show that $x R_{n}^{1+\alpha} y \Rightarrow x R_{n-1}^{e(1+\alpha)} y$. For $n=0$ this is trivial and in case $n \neq 0$ we reason as follows. 
Since $x R_{n}^{1+\alpha} y$ we get in particular that $x_{n} \geq y_{n}+1+\alpha$. We proceed by a case distinction. If $y_{n}=0$, then by Lemma 4.1.19 we see

$$
x_{n-1} \geq y_{n-1}+e\left(x_{n}\right) \geq y_{n-1}+e\left(y_{n}+1+\alpha\right)=y_{n-1}+e(1+\alpha) .
$$

Thus, $x_{n-1} \geq y_{n-1}+\left(1+e\left(y_{n}\right)\right) \cdot(1+e(1+\alpha))$ and by Lemma 4.1.20 we are done. If $y_{n}>0$, then $e\left(y_{n}\right) \in$ Lim and so, using the fact (Lemma 4.1.16) that $e\left(y_{n}+1+\alpha\right)=e\left(y_{n}\right) \cdot e(1+\alpha)$ we get that

$x_{n-1} \geq y_{n-1}+e\left(x_{n}\right) \geq y_{n-1}+e\left(y_{n}+1+\alpha\right)=y_{n-1}+\left(1+e\left(y_{n}\right)\right) \cdot(1+e(1+\alpha))$.

The result now follows from an application of Lemma 4.1.20.

\section{Definable sets}

In this subsection we shall define a map between formulas in MNF and $\ell$-sequences as well as a way of characterizing definable subsets of $I$. Moreover, we shall see how some of these subsets of $I$ can be related to the extensions of single formulas.

In virtue of the reduction axioms (Axiom 5), a formula $\psi \in$ MNF may bear implicit information on monomials $\left\langle n^{\alpha}\right\rangle \top$ for $n \notin \mathrm{N}-\bmod (\psi)$. The next definition is made to retrieve this information.

Definition 4.1.21. Let $\psi:=\left\langle n_{0}^{\alpha_{0}}\right\rangle \top \wedge \ldots \wedge\left\langle n_{k}^{\alpha_{k}}\right\rangle \top \in M N F$. By $\pi_{n_{i}}(\psi)$ we denote the corresponding exponent $\alpha_{i}$. Moreover, for $m \notin N$ - $\bmod (\psi)$, with $n_{k}>m$, $\pi_{m}(\psi)$ is set to be $e\left(\pi_{m+1}(\psi)\right)$ and for $m^{\prime}>n_{k}, \pi_{m^{\prime}}(\psi)$ is defined to be 0 .

We can use these functions to associate a sequence of ordinals to each formula in MNF.

Definition 4.1.22. Let $\psi:=\left\langle n_{0}^{\alpha_{0}}\right\rangle \top \wedge \ldots \wedge\left\langle n_{k}^{\alpha_{k}}\right\rangle \top \in M N F$. By $x_{\psi}$ we denote the sequence $\left\langle\pi_{i}(\psi)\right\rangle_{i<\omega}$.

On account of Definition 4.1.21, we can observe that for $\psi \in M N F$, we have that $x_{\psi} \in I$. Therefore, we shall refer $x_{\psi}$ as the corresponding world of $\psi$. Furthermore, we shall see that $x_{\psi}$ is the first point in $I$ where $\psi$ holds. First we make some simple observations.

\section{Lemma 4.1.23.}

1. For any $x \in I, x \Vdash\left\langle n^{\alpha}\right\rangle \top$ iff $x_{n} \geq \alpha$;

2. For any $\psi \in M N F, x_{\psi} \Vdash \psi$.

Proof. The second item follows from the first one and the definition of $x_{\psi}$ (Definition 4.1.22). For the right-to-left implication of the first item, the case $\alpha=0$ is straightforward. Hence, assume $x_{n} \geq \alpha>0$. Therefore, for $i<n$, we 
have that $x_{i}>0$ and for $i>n, x_{i} \geq 0$. Thus, by the characterization theorem (Theorem 4.1.13), $x R_{n}^{\alpha}\langle 0\rangle$ and so $x \Vdash\left\langle n^{\alpha}\right\rangle \top$.

For the other direction, assume $x \Vdash\left\langle n^{\alpha}\right\rangle \top$ for $\alpha>0$. Hence, there is $y \in I$ such that $x R_{n}^{\alpha} y$ and $y \Vdash \top$. By the characterization theorem, $x_{n} \geq y_{n}+$ $\left(1+e\left(y_{n+1}\right)\right) \cdot \alpha$ and so, $x_{n} \geq \alpha$.

The following two definitions introduce the extension of $\ell$-sequences and the extension of formulas, respectively.

Definition 4.1.24. Given $x \in I$, by $\llbracket x \rrbracket$ we denote the set of $\ell$-sequences which are coordinate-wise at least as big as $x$. That is, we define $\llbracket x \rrbracket:=\{y \in I$ : $y_{i} \geq x_{i}$ for every $\left.i<\omega\right\}$.

Definition 4.1.25. Let $\varphi \in \mathbb{F}_{T S C}$. By $\varphi \rrbracket$ we denote the set of worlds where $\varphi$ holds i.e. $\llbracket \varphi \rrbracket=\{x \in I: x \Vdash \varphi\}$.

The following lemma relates definitions 4.1.24 and 4.1.25.

Lemma 4.1.26. For any $\varphi \in \mathbb{F}_{T S C}$, there is $x:=\left\langle x_{0}, \ldots, x_{k}, 0\right\rangle \in I$ such that $\llbracket \varphi \rrbracket=\llbracket x \rrbracket$.

Proof. The proof goes by induction on $\varphi$. The base case is trivial. For the conjunctive case, let $\varphi=\psi \wedge \chi$. By the I.H. we have that there are $y, z \in I$ such that $\llbracket \psi \rrbracket=\llbracket y \rrbracket$ and $\llbracket \chi \rrbracket=\llbracket z \rrbracket$. Moreover, by the I.H. we also have that $y:=\left\langle y_{0}, \ldots, y_{j}, 0\right\rangle$ and $z:=\left\langle z_{0}, \ldots, z_{k}, 0\right\rangle$. Let $n=\max (j, k)$, that is, the index of the rightmost non-zero component. Hence we can define $x$ as follows:

- $x_{i}=\max \left(y_{i}, z_{i}\right)$ for $i \geq n$;

- $x_{i}=\min \left\{\delta: \delta \geq \max \left(y_{i}, z_{i}\right) \& l(\delta) \geq x_{i+1}\right\}$ for $i<n$.

We can easily check that $x \in I$. Next, we check that for any $x^{\prime} \in I$, we have that

$$
x^{\prime} \Vdash \psi \wedge \chi \text { iff } x^{\prime} \in \llbracket x \rrbracket .
$$

For the right-to-left implication, consider $x^{\prime} \in \llbracket x \rrbracket$. Thus, for $i<\omega$, we have that both $x_{i}^{\prime} \geq x_{k} \geq y_{i}$ and $x_{i}^{\prime} \geq x_{i} \geq z_{i}$. Thus, $x^{\prime} \in \llbracket y \rrbracket \cap \llbracket z \rrbracket$ and so by the I.H. $x^{\prime} \Vdash \psi \wedge \chi$.

For the other direction, consider $x^{\prime} \in I$ such that $x^{\prime} \Vdash \psi \wedge \chi$. Clearly, for $i>n$, we have that $x_{i}^{\prime} \geq x_{i}$. We check by induction on $m$ that $x_{n-m}^{\prime} \geq x_{n-m}$. For the base case, since $x^{\prime} \Vdash \psi \wedge \chi$, then by the I.H. $x^{\prime} \in \llbracket y \rrbracket \cap \llbracket z \rrbracket$ and so $x_{n}^{\prime} \geq y_{n}$ and $x_{n}^{\prime} \geq z_{n}$. Thus, $x_{n}^{\prime} \geq \max \left(y_{n}, z_{n}\right)=x_{n}$. For the inductive step, by definition of the Ignatiev sequences together with the I.H., we have that $l\left(x_{n-(m+1)}^{\prime}\right) \geq x_{n-m}^{\prime} \geq x_{n-m}$ and since $x^{\prime} \Vdash \psi \wedge \chi$, then we can conclude that $x_{n-(m+1)}^{\prime} \geq \max \left(y_{n-(m+1)}, z_{n-(m+1)}\right)$. Therefore, being $x_{n-(m+1)}$ the minimal 
ordinal satisfying both conditions, we can conclude that $x_{n-(m+1)}^{\prime} \geq x_{n-(m+1)}$. Hence, $\llbracket \psi \wedge \chi \rrbracket=\llbracket x \rrbracket$.

For the modality case, let $\varphi:=\left\langle n^{\alpha}\right\rangle \psi$ with $\alpha>0$. Thus, by the I.H. there is $y \in I$ such that $\llbracket \psi \rrbracket=\llbracket y \rrbracket$ and $y:=\left\langle y_{0}, \ldots, y_{j}, 0\right\rangle$. We can define $x$ as follows:

- $x_{i}=y_{i}$ for $i>n$;

- $x_{n}=y_{n}+\left(1+e\left(y_{n+1}\right)\right) \cdot \alpha$;

- $x_{i}=\min \left\{\delta: \delta>y_{i} \& l(\delta) \geq x_{i+1}\right\}$ for $i<n$.

As in the previous case, we can easily check that $x \in I$. Thus, we check that $\llbracket x \rrbracket=\llbracket\left\langle n^{\alpha}\right\rangle \psi \rrbracket$. To prove $\llbracket x \rrbracket \subseteq \llbracket\left\langle n^{\alpha}\right\rangle \psi \rrbracket$, let $x^{\prime} \in \llbracket x \rrbracket$. By the characterization theorem (Theorem 4.1.13) we can see that $x R_{n}^{\alpha} y$. Hence, since $x_{i}^{\prime} \geq x_{i}$ for $i<\omega, x^{\prime} R_{n}^{\alpha} y$ and so $x^{\prime} \Vdash\left\langle n^{\alpha}\right\rangle \psi$.

For the other inclusion, consider $x^{\prime} \in I$ such that $x^{\prime} \Vdash\left\langle n^{\alpha}\right\rangle \psi$. Thus, there is $z \in \llbracket y \rrbracket$ such that $x^{\prime} R_{n}^{\alpha} z$. Hence, with the help of the characterization theorem we can establish that for $i>n$ we have that $x_{i}^{\prime} \geq z_{i} \geq y_{i}=x_{i}$, that is, $x_{i}^{\prime} \geq x_{i}$. For $i \leq n$, we proceed by an easy induction on $k$ to see that $x_{n-k}^{\prime} \geq$ $x_{n-k}$. The base case follows directly from the characterization theorem. For the inductive step, by definition of Ignatiev sequences together with the I.H., we have that $l\left(x_{n-(k+1)}^{\prime}\right) \geq x_{n-k}^{\prime} \geq x_{n-k}$. Since $x^{\prime} \Vdash\left\langle n^{\alpha}\right\rangle \psi$, then there is $z \in I$ such that $x^{\prime} R_{n}^{\alpha} z$ and $z \Vdash \psi$. Thus, by the I.H., $z \in \llbracket y \rrbracket$, and so we have that $x_{n-(k+1)}^{\prime}>z_{n-(k+1)} \geq y_{n-(k+1)}$. Therefore, we get that $l\left(x_{n-(k+1)}^{\prime}\right) \geq x_{n-k}$ and $x_{n-(k+1)}^{\prime}>y_{n-(k+1)}$. Thus, since $x_{n-(k+1)}$ is the least ordinal satisfying both conditions, we have that $x_{n-(k+1)}^{\prime} \geq x_{n-(k+1)}$.

Some of the facts established in the proof of the previous lemma are collected in the following corollary.

Corollary 4.1.27. For any $\varphi, \psi \in \mathbb{F}_{T S C}$ :

1. If $\llbracket\left\langle x_{0}, \ldots, x_{j}, 0\right\rangle \rrbracket=\llbracket \varphi \rrbracket$ and $\llbracket\left\langle y_{0}, \ldots, y_{k}, 0\right\rangle \rrbracket=\llbracket \psi \rrbracket$, then there is $z \in I$ such that $\llbracket z \rrbracket=\llbracket \varphi \wedge \psi \rrbracket$ and $z$ is defined as follows:

- $z_{i}=\max \left(x_{i}, y_{i}\right)$ for $i \geq \max (j, k)$;

- $z_{i}=\min \left\{\delta: \delta \geq \max \left(x_{i}, y_{i}\right) \& l(\delta) \geq z_{i+1}\right\}$ for $i<\max (j, k)$.

2. If $\llbracket x \rrbracket=\llbracket \varphi \rrbracket$, then there is $y \in I$ such that $\llbracket y \rrbracket=\llbracket\left\langle n^{\alpha}\right\rangle \varphi \rrbracket$ and $y$ is defined as follows:

- $y_{i}=x_{i}$ for $i>n$;

- $y_{n}=x_{n}+\left(1+e\left(y_{x+1}\right)\right) \cdot \alpha$;

- $y_{i}=\min \left\{\delta: \delta>x_{i} \& l(\delta) \geq y_{i+1}\right\}$ for $i<n$. 
The next lemma relates the extension of a formula $\psi$ in MNF with the extension of its corresponding world $x_{\psi}$.

Lemma 4.1.28. For any $\psi \in M N F$, we have that $\llbracket \psi \rrbracket=\llbracket x_{\psi} \rrbracket$.

Proof. Let $\psi:=\left\langle n_{0}^{\alpha_{0}}\right\rangle \top \wedge \ldots \wedge\left\langle n_{k}^{\alpha_{k}}\right\rangle \top$. We proceed by induction on $k$. For the base case, we start by checking $\llbracket \psi \rrbracket \subseteq \llbracket x_{\psi} \rrbracket$. Assume $x \Vdash\left\langle n_{0}^{\alpha_{0}}\right\rangle \top$. Then, there is $y \in I$ such that $x R_{n_{0}}^{\alpha_{0}} y$, and so, by the characterization theorem (Theorem 4.1.13) we have that $x R_{m}^{e^{n_{0}-m}\left(\alpha_{0}\right)} y$ for $m \leq n$, that is, $x \Vdash$ $\left\langle m^{e^{n} 0^{-m}}\left(\alpha_{0}\right)\right\rangle T$. Therefore, with the help of Lemma 4.1.23, Item 1 we have that $x_{m} \geq e^{n_{0}-m}\left(\alpha_{0}\right)$, and so $x \in \llbracket x_{\psi} \rrbracket$.

For the other inclusion, let $x \in \llbracket x_{\psi} \rrbracket$. Thus, $x_{n_{0}} \geq\left(x_{\psi}\right)_{n_{0}}$ and so, by Lemma 4.1.23, Item $1 x \Vdash\left\langle n_{0}^{\alpha_{0}}\right\rangle \top$. For the inductive step we reason analogously.

\subsection{Modal Soundness and Completeness}

We dedicate this section to prove the soundness and completeness of TSC w.r.t. $\mathcal{J}$.

\section{Modal soundness}

To prove the soundness of TSC, let us begin by semantically define the entailment between our modal formulas.

Definition 4.2.1. For any formulas $\varphi, \psi \in \mathbb{F}_{T S C}$, we write $\left.\varphi\right|_{\mathcal{J}} \psi$ iff for all $x \in I$, if $x \Vdash \varphi$ then $x \Vdash \psi$. Analogously, we write $\varphi \equiv \mathcal{J} \psi$ iff for any $x \in I$, we have that $x \Vdash \varphi$ iff $x \Vdash \psi$.

With our notion of semantical entailment we can formulate our soundness theorem.

Theorem 4.2.2 (Soundness). For any formulas $\varphi, \psi \in \mathbb{F}_{T S C}$,

$$
\varphi \vdash \psi \Longrightarrow \varphi=_{\mathcal{J}} \psi
$$

Proof. By induction on the length of a TSC proof of $\varphi \vdash \psi$. It is easy to see that the first three rules:

1. If $\varphi \vdash \psi$ and $\varphi \vdash \chi$, then $\varphi \vdash \psi \wedge \chi$;

2. If $\varphi \vdash \psi$ and $\psi \vdash \chi$, then $\varphi \vdash \chi$;

3. If $\varphi \vdash \psi$, then $\left\langle n^{\alpha}\right\rangle \varphi \vdash\left\langle n^{\alpha}\right\rangle \psi$;

preserve validity. With respect to the axioms, the first two axioms

1. $\varphi \vdash \varphi, \varphi \vdash \top$; 
2. $\varphi \wedge \psi \vdash \varphi, \quad \varphi \wedge \psi \vdash \psi$;

are easily seen to be valid. The the correctness of reduction axiom

$$
\left\langle(m+n)^{\alpha}\right\rangle \varphi \vdash\left\langle m^{e^{n}(\alpha)}\right\rangle \varphi
$$

is given by the characterization theorem (Theorem 4.1.13). The remaining axioms and rules are separately proven to be sound in the remainder of this subsection.

We start by proving the soundness of co-additivity axiom i.e.

$$
\left\langle n^{\alpha}\right\rangle\left\langle n^{\beta}\right\rangle \varphi \equiv \mathcal{J}\left\langle n^{\beta+\alpha}\right\rangle \varphi .
$$

Proposition 4.2.3. For any $x, z \in I, n<\omega$ and $\alpha, \beta<\Lambda$,

$$
\exists y \in I\left(x R_{n}^{\alpha} y \text { and } y R_{n}^{\beta} z\right) \Longleftrightarrow x R_{n}^{\beta+\alpha} z .
$$

Proof. We proceed by transfinite induction on $\alpha$ with the base case being trivial. For $\alpha \in$ Succ, let $\alpha:=\delta+1$ for some $\delta$. Therefore:

$$
\begin{aligned}
x R_{n}^{\alpha} y \text { and } y R_{n}^{\beta} z & \Leftrightarrow x R_{n}^{\delta+1} y \text { and } y R_{n}^{\beta} z ; \\
& \Leftrightarrow \exists u\left(x R_{n} u \wedge u R_{n}^{\delta} y \wedge y R_{n}^{\beta} z\right) ; \\
& \Leftrightarrow \exists u\left(x R_{n} u \wedge u R_{n}^{\beta+\delta} z\right), \text { by the I.H. ; } \\
& \Leftrightarrow x R_{n}^{\beta+\delta+1} z ; \\
& \Leftrightarrow x R_{n}^{\beta+\alpha} z .
\end{aligned}
$$

For $\alpha \in \operatorname{Lim}$, we have that $x R_{n}^{\alpha} y$ and $y R_{n}^{\beta} z \Leftrightarrow \forall \delta<\alpha\left(x R_{n}^{1+\delta} y \wedge y R_{n}^{\beta} z\right)$ by Proposition 4.1.8. By the I.H. we obtain $\forall \delta<\alpha x R_{n}^{\beta+1+\delta} z$ and so $x R_{n}^{\beta+\alpha} z$.

With this last result, we get the co-additivity of the $R_{n}^{\alpha}$ relations. This together with Definition 4.1.9 gives us the following corollary.

Corollary 4.2.4. The co-additivity axiom is sound.

Proof. By Definition 4.1.9, $x \Vdash\left\langle n^{\alpha}\right\rangle\left\langle n^{\beta}\right\rangle \varphi$ iff there are $y, z \in I$ such that $x R_{n}^{\alpha} y, y R_{n}^{\beta} z$ and $z \Vdash \varphi$. Thus, by Proposition 4.2.3, $x \Vdash\left\langle n^{\alpha}\right\rangle\left\langle n^{\beta}\right\rangle \varphi$ iff $x R_{n}^{\beta+\alpha} z$ and $z \Vdash \varphi$ i.e., $x \Vdash\left\langle n^{\beta+\alpha}\right\rangle \varphi$.

The following proposition establishes the soundness of the monotonicity axiom.

Proposition 4.2.5. The monotonicity axiom is sound, that is:

$$
\left\langle n^{\alpha}\right\rangle \varphi \models \mathcal{J}\left\langle n^{\beta}\right\rangle \varphi
$$

for $\beta<\alpha$. 
Proof. With the help of the monotonicity property of the $R_{n}^{1+\alpha}$ relations (Lemma 4.1.10, Item 4), we have that if $x \Vdash\left\langle n^{\alpha}\right\rangle \varphi$ then $x \Vdash\left\langle n^{\beta}\right\rangle \varphi$ for $\beta, 0<\beta<\alpha$. We check that if $x \Vdash\left\langle n^{1}\right\rangle \varphi$ then $x \Vdash \varphi$ by induction on $\varphi$.

The base and the conjunctive cases are straightforward, so we consider $\varphi:=\left\langle m^{\delta}\right\rangle \psi$ for some $\delta>0$ and assume $x \Vdash\left\langle n^{1}\right\rangle\left\langle m^{\delta}\right\rangle \psi$. Thus, there are $y, z \in I$ such that $x R_{n} y R_{m}^{\delta} z$ and $z \Vdash \psi$. Then, by the polytransitivity condition (Lemma 4.1.11) we have that $x R_{m}^{\delta} z$, and so $x \Vdash\left\langle m^{\delta}\right\rangle \psi$.

The following proposition establishes the correction of the Schmerl axiom by using the translation between formulas in monomial normal form and $\ell$-sequences.

Proposition 4.2.6. The Schmerl axiom is sound i.e.

$$
\left\langle n^{\alpha}\right\rangle\left(\left\langle n_{0}^{\alpha_{0}}\right\rangle \top \wedge \psi\right) \equiv \mathcal{J}\left\langle n^{e^{n_{0}-n}\left(\alpha_{0}\right) \cdot(1+\alpha)}\right\rangle \top \wedge\left\langle n_{0}^{\alpha_{0}}\right\rangle \top \wedge \psi
$$

for $n<n_{0}$ and $\left\langle n_{0}^{\alpha_{0}}\right\rangle \top \wedge \psi \in M N F$.

Proof. For the left-to-right direction, assume $x \Vdash\left\langle n^{\alpha}\right\rangle\left(\left\langle n_{0}^{\alpha_{0}}\right\rangle \top \wedge \psi\right)$. Thus, by soundness of monotonicity axiom, we have that $x \Vdash\left\langle n_{0}^{\alpha_{0}}\right\rangle \top \wedge \psi$. Therefore, we only need to check that $x \Vdash\left\langle n^{e^{n} 0^{-n}\left(\alpha_{0}\right) \cdot(1+\alpha)}\right\rangle \top$. We can observe that $x \Vdash\left\langle n^{\alpha}\right\rangle\left\langle n_{0}^{\alpha_{0}}\right\rangle \top$ and so there are $y, z \in I$ such that $x R_{n}^{\alpha} y R_{n_{0}}^{\alpha_{0}} z$. By the characterization theorem (Theorem 4.1.13) we have that

$$
x_{n} \geq y_{n}+\left(1+e\left(y_{n+1}\right)\right) \cdot \alpha .
$$

Also since $y R_{n_{0}}^{\alpha_{0}} z$ then $y R_{n}^{e^{n_{0}-n}\left(\alpha_{0}\right)} z$ and $y R_{n+1}^{e^{n_{0}-(n+1)}\left(\alpha_{0}\right)} z$. Hence by the characterization theorem we get that $y_{n} \geq e^{n_{0}-n}\left(\alpha_{0}\right)$ and $y_{n+1} \geq e^{n_{0}-(n+1)}\left(\alpha_{0}\right)$. Combining this with 4.1 we get that

$$
\begin{aligned}
x_{n} & \geq e^{n_{0}-n}\left(\alpha_{0}\right)+\left(1+e\left(e^{n_{0}-(n+1)}\left(\alpha_{0}\right)\right)\right) \cdot \alpha \\
& =e^{n_{0}-n}\left(\alpha_{0}\right)+e\left(e^{n_{0}-(n+1)}\left(\alpha_{0}\right)\right) \cdot \alpha \\
& =e^{n_{0}-n}\left(\alpha_{0}\right)+e^{n_{0}-n}\left(\alpha_{0}\right) \cdot \alpha \\
& =e^{n_{0}-n}\left(\alpha_{0}\right) \cdot(1+\alpha) .
\end{aligned}
$$

Thus, we have that $x R_{n}^{e^{n_{0}-n}\left(\alpha_{0}\right) \cdot(1+\alpha)}\langle 0\rangle$ and so, $x \Vdash\left\langle n^{e^{n_{0}-n}\left(\alpha_{0}\right) \cdot(1+\alpha)}\right\rangle \top$.

For the other direction, assume $x \Vdash\left\langle n^{e^{n_{0}-n}\left(\alpha_{0}\right) \cdot(1+\alpha)}\right\rangle \top \wedge\left\langle n_{0}^{\alpha_{0}}\right\rangle \top \wedge \psi$. Let $\psi^{\prime}=\left\langle n^{e^{n_{0}-n}\left(\alpha_{0}\right) \cdot(1+\alpha)}\right\rangle \top \wedge\left\langle n_{0}^{\alpha_{0}}\right\rangle \top \wedge \psi$. In virtue of Definition 4.1.22 we can consider the corresponding worlds of $\left\langle n_{0}^{\alpha_{0}}\right\rangle \top \wedge \psi$ and $\psi^{\prime}$, that are, $x_{\left\langle n_{0}^{\alpha_{0}}\right\rangle \top \wedge \psi}$ and $x_{\psi^{\prime}}$. We set out to prove $x R_{n}^{\alpha} x_{\left\langle n_{0}^{\alpha_{0}}\right\rangle \top \wedge \psi}$.

Since $x \Vdash \psi^{\prime}$ then by Lemma 4.1.28 we have that $x \in \llbracket \psi^{\prime} \rrbracket=\llbracket x_{\psi^{\prime}} \rrbracket$. Thus, we can make the following observations in order to apply the characterization theorem: 
- By Definition 4.1.22, for all $m>n$, we have that $\left(x_{\psi^{\prime}}\right)_{m}=\left(x_{\left\langle n_{0}^{\alpha}\right\rangle \backslash \wedge \psi}\right)_{m}$. Combining this with the fact that $x \in \llbracket x_{\psi} \rrbracket$, we get that for all $m>n$, $x_{m} \geq\left(x_{\left\langle n_{0}^{\alpha_{0}}\right\rangle \uparrow \wedge \psi}\right)_{m} ;$

- Since $x \in \llbracket x_{\psi} \rrbracket$ we have that $x_{n} \geq\left(x_{\psi^{\prime}}\right)_{n}=e^{n_{0}-n}\left(\alpha_{0}\right) \cdot(1+\alpha)$. Also, by Definition 4.1.22, we can observe that $\left(x_{\left\langle n_{0}^{\alpha_{0}}\right\rangle \top \wedge \psi}\right)_{n}=e^{n_{0}-n}\left(\alpha_{0}\right)$ and $\left(x_{\left\langle n_{0}^{\alpha_{0}}\right\rangle \uparrow \wedge \psi}\right)_{n+1}=e^{n_{0}-(n+1)}\left(\alpha_{0}\right)$. Thus, we can reason as follows:

$$
\begin{aligned}
x_{n} & \geq e^{n_{0}-n}\left(\alpha_{0}\right) \cdot(1+\alpha) \\
& =e^{n_{0}-n}\left(\alpha_{0}\right)+e^{n_{0}-n}\left(\alpha_{0}\right) \cdot \alpha \\
& =e^{n_{0}-n}\left(\alpha_{0}\right)+e\left(e^{n_{0}-(n+1)}\left(\alpha_{0}\right)\right) \cdot \alpha \\
& =\left(x_{\left\langle n_{0}^{\alpha_{0}}\right\rangle \top \wedge \psi}\right)_{n}+\left(1+e\left(\left(x_{\left\langle n_{0}^{\alpha_{0}}\right\rangle \top \wedge \psi}\right)_{n+1}\right)\right) \cdot \alpha ;
\end{aligned}
$$

- For all $m<n$, we can observe that $x_{n} \geq e^{n-m}\left(e^{n_{0}-n}\left(\alpha_{0}\right) \cdot(1+\alpha)\right)$. On the other hand, $\left(x_{\left\langle n_{0}^{\alpha_{0}}\right\rangle \uparrow \wedge \psi}\right)_{m}=e^{n-m}\left(e^{n_{0}-n}\left(\alpha_{0}\right)\right)$. Therefore, for all $m<n$, we have that $x_{m}>\left(x_{\left\langle n_{0}^{\alpha_{0}}\right\rangle \top \wedge \psi}\right)_{m}$.

Hence, by the characterization theorem, we have that $x R_{n}^{\alpha} x_{\left\langle n_{0}^{\alpha_{0}}\right\rangle \top \wedge \psi^{\prime}}$ and thus $x_{\left\langle n_{0}^{\alpha_{0}}\right\rangle \top \wedge \psi}$.

Lastly, we check the soundness of Rule 4 by applying the relation between definable sets and the extension of $\ell$-sequences proved in Lemma 4.1.26. This next result concludes the soundness proof of TSC.

Proposition 4.2.7. If $\varphi \models \mathcal{J} \psi$ then, for $m<n$ :

$$
\left\langle n^{\alpha}\right\rangle \varphi \wedge\left\langle m^{\beta+1}\right\rangle \psi \models_{\mathcal{J}}\left\langle n^{\alpha}\right\rangle\left(\varphi \wedge\left\langle m^{\beta+1}\right\rangle \psi\right) .
$$

Proof. Assume $\varphi \models_{\mathcal{J}} \psi$ and let $x \in I$ such that $x \Vdash\left\langle n^{\alpha}\right\rangle \varphi \wedge\left\langle m^{\beta+1}\right\rangle \psi$. Since $\varphi=\mathcal{J} \psi$, by Lemma 4.1.26, there are $y, z \in I$ such that

$$
\llbracket y \rrbracket=\llbracket \varphi \rrbracket \subseteq \llbracket \psi \rrbracket=\llbracket z \rrbracket .
$$

Let $y^{\prime}, z^{\prime} \in I$ such that $\llbracket y^{\prime} \rrbracket=\llbracket\left\langle n^{\alpha}\right\rangle \varphi \rrbracket$ and $\llbracket z^{\prime} \rrbracket=\llbracket\left\langle m^{\beta+1}\right\rangle \psi \rrbracket$, and $w \in I$ such that:

$$
\llbracket w \rrbracket=\llbracket \varphi \wedge\left\langle m^{\beta+1}\right\rangle \psi \rrbracket=\llbracket \varphi \rrbracket \cap \llbracket\left\langle m^{\beta+1}\right\rangle \psi \rrbracket=\llbracket y \rrbracket \cap \llbracket z^{\prime} \rrbracket .
$$

First, we shall make some observations about $w$ and $x$, respectively. We will use these facts together with the characterization theorem (Theorem 4.1.13) to conclude that $x R_{n}^{\alpha} w$. Since $y \in \llbracket z \rrbracket$, then for all $i<\omega$ we have that $y_{i} \geq z_{i}$. Furthermore, by Corollary 4.1.27, Item 2 we have that for $i>m, z_{i}^{\prime}=z_{i}$. Thus, we can observe that

- For $i>m$ we have that 


$$
\begin{aligned}
w_{i} & =\min \left\{\delta: \delta \geq \max \left(y_{i}, z_{i}^{\prime}\right) \& l(\delta) \geq w_{i+1}\right\} \\
& =\min \left\{\delta: \delta \geq \max \left(y_{i}, z_{i}\right) \& l(\delta) \geq w_{i+1}\right\} \\
& =y_{i} ; \\
\text { - } w_{m} & =\min \left\{\delta: \delta \geq \max \left(y_{i}, z_{i}^{\prime}\right) \& l(\delta) \geq w_{m+1}\right\} \\
& =\min \left\{\delta: \delta \geq \max \left(y_{i}, z_{i}^{\prime}\right) \& l(\delta) \geq y_{m+1}\right\} .
\end{aligned}
$$

On the other hand, since $x \Vdash\left\langle n^{\alpha}\right\rangle \varphi \wedge\left\langle m^{\beta+1}\right\rangle \psi$, we have the following:

- By Corollary 4.1.27, Item I $x_{i} \geq \min \left\{\delta: \delta \geq \max \left(y_{i}^{\prime}, z_{i}^{\prime}\right) \& l(\delta) \geq x_{i+1}\right\}$ for $i \leq m$;

- Since $x \Vdash\left\langle n^{\alpha}\right\rangle \varphi \wedge\left\langle m^{\beta+1}\right\rangle \psi$, then $x \Vdash \varphi$ and so, we have that $x_{i} \geq y_{i}$ for $i>n$;

- As $x \Vdash\left\langle n^{\alpha}\right\rangle \varphi$ we get that $x_{n} \geq y_{n}^{\prime}$ and $x_{i} \geq \min \left\{\delta: \delta \geq y_{i}^{\prime} \& l(\delta) \geq x_{i+1}\right\}$ for $i, m<i<n$.

It remains to be checked that $x R_{n}^{\alpha} w$. Recall that $m<n$ and that for $i>m$, we have that $w_{i}=y_{i}$. Thus, for $i>n$, we have that $x_{i} \geq y_{i}=w_{i}$. Also, since $w_{n}=y_{n}, w_{n+1}=y_{n+1}$ and $x_{n} \geq y_{n}^{\prime}=y_{n}+\left(1+e\left(y_{n+1}\right)\right) \cdot \alpha$ we have that $x_{n} \geq w_{n}+\left(1+e\left(w_{n+1}\right)\right) \cdot \alpha$. Thus, we need to see that $x_{i}>w_{i}$ for $i<n$.

For $i, m<i<n$, we know that $y_{i}=w_{i}$ and by Corollary 4.1.27, Item 2, $y_{i}^{\prime}>y_{i}$. Since $x_{i} \geq y_{i}^{\prime}$ for $i, m<i<n$, then $x_{i}>w_{i}$. For $i \leq m$, we show by induction on $k$ that $x_{m-k}>w_{m-k}$. For the base case, recall that by assumption $\varphi=_{\mathcal{J}} \psi$ and so, $y \in \llbracket z \rrbracket$, that is, for $i \in \omega, y_{i} \geq z_{i}$. Thus, we can first observe that $z_{m+1} \leq y_{m+1}=w_{m+1}<x_{m+1}$ and $\max \left(y_{m}^{\prime}, z_{m}^{\prime}\right) \geq \max \left(y_{m}, z_{m}^{\prime}\right)$. We make the following case distinction:

- If $w_{m}=y_{m}$, then $x_{m} \geq y_{m}^{\prime}>y_{m}$. Therefore, $x_{m}>w_{m}$;

- If $w_{m}=z_{m}+\left(1+e\left(z_{m+1}\right)\right) \cdot(1+\beta)+e\left(w_{m+1}\right)$, then since $x_{m+1}>w_{m+1}$, we have that

$$
\begin{aligned}
x_{m} & \geq z_{m}+\left(1+e\left(z_{m+1}\right)\right) \cdot(1+\beta)+e\left(x_{m+1}\right) \\
& >z_{m}+\left(1+e\left(z_{m+1}\right)\right) \cdot(1+\beta)+e\left(w_{m+1}\right) \\
& =w_{m} .
\end{aligned}
$$

Thus, $x_{m}>w_{m}$.

For the inductive step, by the I.H. we have that $x_{m-k}>w_{m-k}$. We reason as follows:

- If $w_{m-(k+1)}=\min \left\{\delta: \delta \geq y_{m-(k+1)} \& l(\delta) \geq w_{m-k}\right\}$, then since $y_{m}^{\prime}>y_{m}$ and by the I.H. we have that $x_{m-(k+1)}>w_{m-(k+1)}$; 
- If $w_{m-(k+1)}=\min \left\{\delta: \delta \geq z_{m-(k+1)}^{\prime} \& l(\delta) \geq w_{m-k}\right\}$, then we can observe that $w_{m-(k+1)}=z_{m-(k+1)}+\left(1+e\left(z_{m-k}\right)\right) \cdot(1+\beta)+e\left(w_{m-k}\right)$. Thus, by the I.H. we get that

$$
\begin{aligned}
x_{m-(k+1)} & \geq z_{m-(k+1)}+\left(1+e\left(z_{m-k}\right)\right) \cdot(1+\beta)+e\left(x_{m-k}\right) \\
& >z_{m-(k+1)}+\left(1+e\left(z_{m-k}\right)\right) \cdot(1+\beta)+e\left(w_{m-k}\right) \\
& =w_{m-(k+1)}
\end{aligned}
$$

and so $x_{m-(k+1)}>w_{m-(k+1)}$.

Hence, in virtue of the characterization theorem we get that $x R_{n}^{\alpha} w$, and so we obtain that $x \Vdash\left\langle n^{\alpha}\right\rangle\left(\varphi \wedge\left\langle m^{\beta+1}\right\rangle \psi\right)$ as needed.

\section{Modal completeness}

In the previous chapter we made use of Corollary 3.3.1 to characterize the non-derivability in our system. As we mentioned, the same result can be obtained by using relational semantics since for any $\alpha>0$ and $x \in I$, we have that $\neg x R_{n}^{\alpha} x$.

In this chapter we shall follow a different approach to establish the modal completeness of TSC. First we prove the following proposition that characterizes the derivability between formulas in monomial normal form in terms of the $\pi_{n}$-functions introduced in Definition 4.1.21. Therefore, this result provides a refinement of Proposition 3.3.11 by means of the $\pi_{n}$-functions.

Proposition 4.2.8. Given $\psi_{0}, \psi_{1} \in M N F$, we have that:

$$
\psi_{0} \vdash \psi_{1} \Longleftrightarrow \pi_{n}\left(\psi_{0}\right) \geq \pi_{n}\left(\psi_{1}\right) \text { for all } n \in N-\bmod \left(\psi_{1}\right) \text {. }
$$

Proof. For the left-to-right implication, assume $\psi_{0} \vdash \psi_{1}$. By Theorem 3.3.11, Item 2 we have that $\pi_{n}\left(\psi_{1}\right) \leq e^{m-n}\left(\pi_{m}\left(\psi_{0}\right)\right)$ for $m=\mu\left(n, \psi_{0}\right)$. We can observe that $e^{m-n}\left(\pi_{m}\left(\psi_{0}\right)\right) \leq \pi_{n}\left(\psi_{0}\right)$, and so, we get that $\pi_{n}\left(\psi_{0}\right) \geq \pi_{n}\left(\psi_{1}\right)$.

For the right-left-implication, assume that $\pi_{n}\left(\psi_{0}\right) \geq \pi_{n}\left(\psi_{1}\right)$ for for any $n \in \mathrm{N}-\bmod \left(\psi_{1}\right)$. We make the following case distinction:

- If $n \in \mathrm{N}-\bmod \left(\psi_{0}\right)$, then we have that $\psi_{0} \vdash\left\langle n^{\pi_{n}\left(\psi_{0}\right)}\right\rangle \top$. Since by assumption $\pi_{n}\left(\psi_{0}\right) \geq \pi_{n}\left(\psi_{1}\right)$, then by monotonicity axiom (Axiom 3), $\left\langle n^{\pi_{n}\left(\psi_{0}\right)}\right\rangle \top \vdash\left\langle n^{\pi_{n}\left(\psi_{1}\right)}\right\rangle \top$. Thus, $\psi_{0} \vdash\left\langle n^{\pi_{n}\left(\psi_{1}\right)}\right\rangle \top$.

- If $n \notin \mathrm{N}-\bmod \left(\psi_{0}\right)$, then $\pi_{n}\left(\psi_{0}\right)=e^{m-n}\left(\pi_{m}\left(\psi_{0}\right)\right)$ where $m=\mu\left(n, \psi_{0}\right)$. Hence, we have that $\psi_{0} \vdash\left\langle n^{\pi_{n}\left(\psi_{0}\right)}\right\rangle \top$ and by reduction axiom (Axiom 5), $\psi_{0} \vdash\left\langle m^{e^{m-n}}\left(\pi_{n}\left(\psi_{0}\right)\right)\right\rangle \top$. Since $e^{m-n}\left(\pi_{m}\left(\psi_{0}\right)\right)=\pi_{n}\left(\psi_{0}\right) \geq \pi_{n}\left(\psi_{1}\right)$, with the help of monotonicity axiom, we get that

$$
\left\langle n^{\pi_{n}\left(\psi_{0}\right)}\right\rangle \top \vdash\left\langle n^{\pi_{n}\left(\psi_{1}\right)}\right\rangle \top .
$$

Therefore, $\psi_{0} \vdash\left\langle n^{\pi_{n}\left(\psi_{1}\right)}\right\rangle \top$. 
Thus, for each monomial $\left\langle n^{\alpha}\right\rangle \top$ occurring in $\psi_{1}$ we have that $\psi_{0} \vdash\left\langle n^{\alpha}\right\rangle \top$, and so, $\psi_{0} \vdash \psi_{1}$.

The following corollary follows immediately from this characterization of the derivability between formulas in monomial normal form.

Corollary 4.2.9. Given $\varphi, \psi \in M N F$, if $\varphi \nvdash \psi$ then there is $m_{I} \in N$ - $\bmod (\psi)$ such that $\pi_{m_{I}}(\varphi)<\pi_{m_{I}}(\psi)$.

Now we are ready to prove the completeness of TSC.

Theorem 4.2.10 (Completeness). Given formulas $\varphi, \psi \in \mathbb{F}_{T S C}$, if $\varphi \models \mathcal{J} \psi$, then $\varphi \vdash \psi$.

Proof. By Theorem 3.3.7, w.l.o.g. let $\varphi, \psi \in$ MNF such that $\varphi:=\left\langle n_{0}^{\alpha_{0}}\right\rangle \top \wedge$ $\ldots \wedge\left\langle n_{k}^{\alpha_{k}}\right\rangle \top$ and $\psi:=\left\langle m_{0}^{\beta_{0}}\right\rangle \top \wedge \ldots \wedge\left\langle m_{j}^{\beta_{j}}\right\rangle \top$. Reasoning by contraposition, suppose $\varphi \nvdash \psi$. Therefore, by Corollary 4.2.9, we can conclude that for some $m_{I} \in \mathrm{N}-\bmod (\psi)$, we have that $\pi_{m_{I}}(\varphi)<\pi_{m_{I}}(\psi)$. Thus, consider the Ignatiev sequence $x_{\varphi}$. By Lemma 4.1.23, Item (2), $x_{\varphi} \Vdash \varphi$ but $x_{\varphi} \Vdash\left\langle m_{I}^{\beta_{I}}\right\rangle \top$. Hence, $x_{\varphi} \| \psi \psi$ and so $\varphi \not \models \mathcal{J} \psi$. 


\section{More on TSC}

We dedicate this chapter to collecting some miscellaneous results about TSC. First, we shall consider a new universal frame for TSC by restricting the domain of $\mathcal{J}$ to those $\ell$-sequences with finite support. This new frame will provide us with some useful arithmetical applications by depicting a road map to consistency strength and conservativity results of Turing progressions. Secondly, we shall introduce a presentation of TSC that makes no use of MNF's, namely TSC*. We prove the equivalence between both systems. Finally, we give a modal formulation of the Schmerl's formulas and provide a modal proof of them without requiring the completeness result.

\subsection{A Finitely Supported Universal Frame}

In this section we present a new universal frame $\mathcal{H}$, which is a slight modification of $\mathcal{J}$ and allows for the modal definability of each world in the domain. More precisely, the set of worlds of $\mathcal{H}$ is built from finitely supported $\ell$-sequences, i.e., sequences of the form $\left\langle x_{0}, \ldots, x_{k}, 0\right\rangle \in I$.

Definition 5.1.1. $\mathcal{H}:=\left\langle H,\left\{S_{n}\right\}_{n<\omega}\right\rangle$ is defined as follows:

$$
H:=\left\{x \in I: \exists n<\omega x_{n}=0\right\}
$$

and

$$
x S_{n} y: \Leftrightarrow\left(\forall m \leq n x_{m}>y_{m} \wedge \forall m>n x_{m} \geq y_{m}\right) .
$$

Remark 5.1.2. Since $H \subseteq I$ and $S_{n}=R_{n} \uparrow H$, clearly for any $x, y \in H$, we have that $x S_{n} y \Longleftrightarrow x R_{n} y$. Moreover, we can easily check that if $x \in H$ and $x R_{n} y$ for some $y \in I$, then $y \in H$ and $x S_{n} y$. 
The next lemma is a reformulation of Theorem 4.1.4 for the $S_{n}$-relations. These observations confirm that the $S_{n}$-relations are good to model provability logic.

\section{Lemma 5.1.3.}

1. Each $S_{n}$ for $n \in \omega$ is transitive: $x S_{n} y \wedge y S_{n} z \Rightarrow x S z$;

2. Each $S_{n}$ for $n \in \omega$ is Noetherian: each non-empty $X \subseteq H$ has an $S_{n^{-}}$ maximal element $y \in X$, i.e., $\forall x \in X \neg y S_{n} x$;

3. The relations $S_{n}$ are monotone in $n$ in the sense that: $x S_{n} y \Rightarrow x S_{m} y$ whenever $n>m$.

As we did for $\mathcal{J}$, we need to define the auxiliary relations $S_{n}^{\alpha}$ for any $n<\omega$ and $\alpha<\Lambda$ whose purpose is to model the $\left\langle n^{\alpha}\right\rangle$ modality.

Definition 5.1.4. Given $x, y \in H$ and $S_{n}$ on $H$, we recursively define $x S_{n}^{\alpha} y$ as follows:

1. $x S_{n}^{0} y \quad: \Leftrightarrow x=y$

2. $x S_{n}^{1+\alpha} y: \Leftrightarrow \forall \beta<1+\alpha \exists z\left(x S_{n} z \wedge z S_{n}^{\beta} y\right)$.

We can also reformulate Definition 4.1.9 in terms of $\mathcal{H}$.

Definition 5.1.5. Let $x \in H$ and $\varphi \in \mathbb{F}_{T S C}$. By $x \Vdash \varphi$ we denote the validity of $\varphi$ in $x$ that is recursively defined as follows:

1. $x \Vdash \top$ for all $x \in H$;

2. $x \Vdash \varphi \wedge \psi$ iff $x \Vdash \varphi$ and $x \Vdash \psi$;

3. $x \Vdash\left\langle n^{\alpha}\right\rangle \varphi$ iff there is $y \in H, x S_{n}^{\alpha} y$ and $y \Vdash \varphi$.

The following proposition establishes the preservation between the $S_{n}^{\alpha}$ relations of $\mathcal{H}$ and the $R_{n}^{\alpha}$ relations of $\mathcal{J}$ when restricted to the elements in $H$.

Proposition 5.1.6. For any $x, y \in H, n<\omega$ and $\alpha<\Lambda$ :

$$
x S_{n}^{\alpha} y \Longleftrightarrow x R_{n}^{\alpha} y .
$$

Proof. We proceed by induction on $\alpha$ being the base case trivial. The successor and limit cases follows from Remark 5.1.2 and the I.H. 


\section{Preservation of validity}

To prove the completeness of TSC w.r.t. $\mathcal{H}$, first we shall prove the preservation of validity between the frames $\mathcal{H}$ and $\mathcal{J}$ when restricted to $\ell$-sequences with finite support.

Theorem 5.1.7. For any $x \in H$ and $\varphi \in \mathbb{F}_{T S C}$,

$$
\mathcal{J}, x \Vdash \varphi \Longleftrightarrow \mathcal{H}, x \Vdash \varphi .
$$

Proof. Proof goes by induction on $\varphi$. The base and conjunctive cases are straightforward. For the case $\varphi:=\left\langle n^{\alpha}\right\rangle \psi$, assume $\mathcal{J}, x \Vdash\left\langle n^{\alpha}\right\rangle \psi$. Thus, there is $y \in I$ such that $x R_{n}^{\alpha} y$ and $\mathcal{J}, y \Vdash \psi$. By Remark 5.1.2, we know $y \in H$ and by Proposition 5.1.6 we have that $x S_{n}^{\alpha} y$. This together with the I.H. gives us that there is $y \in H$ such that $x S_{n}^{\alpha} y$ and $\mathcal{H}, y \Vdash \psi$, that is, $\mathcal{H}, x \Vdash\left\langle n^{\alpha}\right\rangle \psi$. For the other implication, suppose that $\mathcal{H}, x \Vdash\left\langle n^{\alpha}\right\rangle \psi$ i.e. there is $y \in H$ such that $x S_{n}^{\alpha} y$ and $\mathcal{H}, y \Vdash \psi$. By Remark 5.1.2, we know $H \subset I$. Thus, by Proposition 5.1.6 and the I.H. we obtain that there is $y \in I$ such that $x R_{n}^{\alpha} y$ and $\mathcal{J}, y \Vdash \psi$ and so, $\mathcal{J}, x \Vdash\left\langle n^{\alpha}\right\rangle \psi$.

Now we can show the completeness of our system w.r.t. $\mathcal{H}$.

Theorem 5.1.8. For any $\varphi, \psi \in \mathbb{F}_{T S C}$, we have that:

$$
\varphi \vdash \psi \Longleftrightarrow \forall x \in H(\mathcal{H}, x \Vdash \varphi \Longrightarrow \mathcal{H}, x \Vdash \psi) .
$$

Proof. For the left-to-right implication, assume $\varphi \vdash \psi$. Thus, by Theorem 4.2.2, $\varphi \models \mathcal{J} \psi$. Assume $\mathcal{H}, x \Vdash \varphi$ for some $x \in H$. Then, by Theorem 5.1.7, $\mathcal{J}, x \Vdash \varphi$ and since $\varphi \models \mathcal{J} \psi$, we get that $\mathcal{J}, x \Vdash \psi$. Hence, by Theorem 5.1.7, $\mathcal{H}, x \Vdash \psi$.

For the other implication, reasoning by contraposition, assume $\varphi \nvdash \psi$ with $\varphi, \psi \in$ MNF. Thus, by Corollary 4.2.9, we have that $\pi_{m}(\varphi)<\pi_{m}(\psi)$ for some $m \in \mathrm{N}-\bmod (\psi)$. Therefore, by Lemma 4.1.23, Item 1 , we have the following:

$$
\mathcal{J}, x_{\varphi} \Vdash \varphi \text { and } \mathcal{J}, x_{\varphi} \Vdash \psi \text {. }
$$

By Definitions 4.1.22 and 4.1.21 we can observe that $x_{\varphi}$ is a $\ell$-sequence with finite support. Thus, $x_{\varphi} \in H$. Hence, by means of Theorem 5.1.7, we obtain that $\mathcal{H}, x_{\varphi} \Vdash \varphi$ but $\mathcal{H}, x_{\varphi} \Vdash \psi$ which concludes the proof.

\section{Modal definability}

In this subsection we shall see an application of the completeness of TSC w.r.t $\mathcal{H}$. As established by some results proven in the previous chapter, some $\ell$-sequences seemed to be modally definable, but having finite support was 
essential. We show how this modal definability can be achieved in $\mathcal{H}$.

By $m: H \rightarrow \mathbb{F}_{\text {TSC }}$ we denote the map defined as follows:

$$
m\left(\left\langle\alpha_{0}, \ldots, \alpha_{j}, 0\right\rangle\right)=\bigwedge_{0 \leq i \leq j}\left\langle i^{\alpha_{i}}\right\rangle \top .
$$

While it is not generally true that given $x \in H$, we have that $m(x) \in \mathrm{MNF}$, by simple applications of the reduction axiom (Axiom 5) we can omit the superfluous terms contained in $m(x)$ and thus, obtain a formula $\psi_{x}$ such that

$$
m(x) \equiv \psi_{x} \in \mathrm{MNF} .
$$

We shall refer to $\psi_{x}$ as the corresponding MNF of $x$. The following lemma establishes the natural relation between the notion of corresponding world, introduced in Definition 4.1.22, and the notion of corresponding MNF. The proof follows immediately from said definition.

Lemma 5.1.9. For any $x \in H$ we have that $x_{\psi_{x}}=x$.

With the following auxiliary definition we are ready to introduce our result about the modal definability of the worlds in $\mathcal{H}$.

Definition 5.1.10. For each $x \in H$, by $x \downarrow$ we denote the subset of $H$ such that $y \in x \downarrow$ iff $y_{n}<x_{n}$ for some $n<\omega$.

The following two results express the relation between the downset generated by a world $x$ and the extension of its corresponding MNF.

Lemma 5.1.11. For any $x, y \in H$ :

$$
y \in x^{\downarrow} \Longleftrightarrow \mathcal{H}, y \| \psi_{x} .
$$

Proof. For the left-to-right implication, assume $y \in x^{\downarrow}$, that is, $y_{n}<x_{n}$ for some $n<\omega$. Thus, by Lemma 4.1.23, Item 1 together with Theorem 5.1.7, we have that $\mathcal{H}, y \Downarrow\left\langle n^{x_{n}}\right\rangle \top$. On the other hand, since $\psi_{x} \equiv m(x)$ and $m(x) \vdash$ $\left\langle n^{x_{n}}\right\rangle \top$, we have that $\psi_{x} \vdash\left\langle n^{x_{n}}\right\rangle \top$. Thus, by completeness (Theorem 5.1.8) we get that $\mathcal{H}, y \| \psi_{x}$.

For the other implication, reasoning by contraposition, assume $y \notin x^{\downarrow}$. Thus, for all $n<\omega$ we have that $y_{n} \geq x_{n}$ and so, in particular, for all $n \in$ $\mathrm{N}-\bmod \left(\psi_{x}\right)$, we have that $y_{n} \geq x_{n}=\pi_{n}\left(\psi_{x}\right)$. Then, by Lemma 4.1.23, Item 1 and Theorem 5.1.7, $\mathcal{H}, y \Vdash \psi_{x}$.

With this last lemma we obtain the following corollary about the modal definability.

Corollary 5.1.12. For any $x \in H$ :

$$
\mathcal{H}, x \Vdash \psi_{x} \& \forall y \in x^{\downarrow}, \mathcal{H}, y \| \psi_{x} .
$$


Proof. Folows directly from previous lemma by observing that $x \notin x^{\downarrow}$.

One of the most useful features of the modal definability of $\mathcal{H}$ is that it provides us with a road map to understand the inclusions that hold between different Turing progressions. The relation between Turing progressions and $\ell$-sequences has already been explored in [32] where Ignatiev's universal frame is presented as a map to conservation results. However, the analysis we provide here allows us to compare both the conservation and the consistency strength of the Turing progressions depicted in the frame (see Figure 5.1).

To capture this idea, first we make the following observation.

Lemma 5.1.13. For any $x, y \in H, n<\omega$ and $\alpha<\Lambda$, we have that:

$$
x S_{n}^{\alpha} y \quad \Longleftrightarrow \quad \psi_{x} \vdash\left\langle n^{\alpha}\right\rangle \psi_{y} .
$$

Proof. For the left-to-right implication, assume $x S_{n}^{\alpha} y$ and let $\mathcal{H}, z \Vdash \psi_{x}$ for some $z \in H$. We set out to prove $\mathcal{H}, z \Vdash\left\langle n^{\alpha}\right\rangle \psi_{y}$. If $\mathcal{H}, z \Vdash \psi_{x}$, then $z \in \llbracket \psi_{x} \rrbracket$. By Lemma 4.1.28, we have that $z \in \llbracket x_{\psi_{x}} \rrbracket$ and by Lemma 5.1.9, $z \in \llbracket x \rrbracket$. Thus, since $x S_{n}^{\alpha} y$ we have that $z S_{n}^{\alpha} y$ and by Theorem 5.1.12, we have that $\mathcal{H}, y \Vdash \psi_{y}$. Therefore, $\mathcal{H}, z \Vdash\left\langle n^{\alpha}\right\rangle \psi_{y}$, and so, by completeness (Theorem 5.1.8), $\psi_{x} \vdash\left\langle n^{\alpha}\right\rangle \psi_{y}$.

For the other implication, assume $\psi_{x} \vdash\left\langle n^{\alpha}\right\rangle \psi_{y}$. By completeness we have that

$$
\forall z \in H\left(\mathcal{H}, z \Vdash \psi_{x} \Longrightarrow \mathcal{H}, z \Vdash\left\langle n^{\alpha}\right\rangle \psi_{y}\right)
$$

and by Theorem 5.1.12, $\mathcal{H}, x \Vdash \psi_{x}$. Hence, $\mathcal{H}, x \Vdash\left\langle n^{\alpha}\right\rangle \psi_{y}$, that is, $\exists z^{\prime} \in H$ such that $x S_{n}^{\alpha} z^{\prime}$ and $\mathcal{H}, z^{\prime} \Vdash \psi_{y}$. Then, $x \in \llbracket z^{\prime} \rrbracket$ and by a combination of Lemmas 4.1.28 and 5.1.9, $z^{\prime} \in \llbracket \psi_{y} \rrbracket=\llbracket y \rrbracket$. Therefore, $x S_{n}^{\alpha} y$.

This last result together with the arithmetical completeness (Theorems 3.2.6 and 3.3.13) gives us the aforementioned road map.

Lemma 5.1.14. For any $x, y \in H, n<\omega$ and $\alpha<\Lambda$, we have that:

$$
x S_{n}^{\alpha} y \Longleftrightarrow \mathrm{EA}^{+} \vdash\left(T h_{\psi_{y}}\right)_{n}^{\alpha} \subseteq T h_{\psi_{x}} .
$$

$\mathcal{H}$ also provide us with a road map to conservation results. This can be achieved by defining the following family of relations $\left\{\approx_{n}\right\}_{n<\omega}$ on $H$ :

$$
x \approx_{n} y: \Leftrightarrow \forall m \leq n x_{m}=y_{m} .
$$

From an arithmetical point of view, the $\approx_{n}$-relations specify that (the theories of) $x$ and $y$ accumulate the same amount of $m$-consistency for $m \leq n$. Thus, we obtain the following result 
Proposition 5.1.15. For any $x, y \in H$ and $n<\omega$, we have that:

$$
x \approx_{n} y \Longleftrightarrow \mathrm{EA}^{+} \vdash T h_{\psi_{x}} \equiv_{n} T h_{\psi_{y}} .
$$

The proof of this last proposition follows from Lemma 3.2.14 and the following observation:

Lemma 5.1.16. Let $\psi:=\left\langle n_{0}^{\alpha_{0}}\right\rangle \top \wedge \ldots \wedge\left\langle n_{k}^{\alpha_{k}}\right\rangle \top \wedge\left\langle m^{\beta}\right\rangle \top \in M N F$ and $n, n_{k}<n<m$. Provably in $\mathrm{EA}^{+}$:

$$
T h_{\psi} \equiv_{n}\left(\mathrm{EA}^{+}\right)_{n_{0}}^{\alpha_{0}}+\ldots+\left(\mathrm{EA}^{+}\right)_{n_{k}}^{\alpha_{k}}+\left(\mathrm{EA}^{+}\right)_{n}^{e^{m-n}(\beta)} .
$$

Proof. Since $\psi \vdash\left\langle n_{0}^{\alpha_{0}}\right\rangle \top \wedge \ldots \wedge\left\langle n_{k}^{\alpha_{k}}\right\rangle \top \wedge\left\langle n^{e^{m-n}(\beta)}\right\rangle \top$, in particular we have the right-to-left inclusion. For the left-to-right inclusion, reasoning in $\mathrm{EA}^{+}$, let $\pi \in \Pi_{n+1}^{0}$ such that

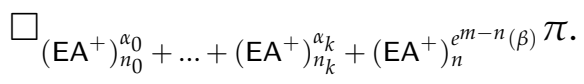

By the formalized deduction theorem we get that

$$
\square_{\left(\mathrm{EA}^{+}\right)_{n}^{e^{m-n}(\beta)}}\left(\bigwedge_{0 \leq i \leq k} \operatorname{Con}_{n_{i}}\left(\left(\mathrm{EA}^{+}\right)_{n_{i}}^{\beta_{i}}\right) \rightarrow \pi\right)
$$

for some sequence of $\beta_{i} \prec \alpha_{i}$. Since each $\operatorname{Con}_{n_{i}}\left(\left(\mathrm{EA}^{+}\right)_{n_{i}}^{\beta_{i}}\right) \in \Pi_{n_{i}+1}^{0}$ we have that $\left(\Lambda_{0 \leq i<j^{\prime}} \operatorname{Con}_{n_{i}}\left(\left(\mathrm{EA}^{+}\right)_{n_{i}}^{\beta_{i}}\right) \rightarrow \pi\right) \in \Pi_{n+1}^{0}$. Thus, by reduction property (Item 3.2.5 of Proposition 3.2.5), we have that

$$
\square_{\left(\mathrm{EA}^{+}\right)_{m}^{\beta}}\left(\bigwedge_{0 \leq i \leq k} \operatorname{Con}_{n_{i}}\left(\left(\mathrm{EA}^{+}\right)_{n_{i}}^{\beta_{i}}\right) \rightarrow \pi\right)
$$

and so, by monotonicity $\square_{\mathrm{Th}_{\psi}} \pi$.

Now we are ready to give a detailed proof of Proposition 5.1.15.

Proof. We start by the left-to-right implication. Suppose $x \approx_{n} y$, and let $\psi_{x}:=\left\langle n_{0}^{\alpha_{0}}\right\rangle \top \wedge \ldots \wedge\left\langle n_{k}^{\alpha_{k}}\right\rangle \top$ and $\psi_{y}:=\left\langle m_{0}^{\beta_{0}}\right\rangle \top \wedge \ldots \wedge\left\langle m_{j}^{\beta_{j}}\right\rangle \top$. Moreover, let $n^{*}=\mu\left(n, \psi_{x}\right)$ and $m^{*}=\mu\left(n, \psi_{y}\right)$. Thus, by a combination of Lemmas 3.2.14 and 5.1.16, we have that:

a) $\mathrm{Th}_{\psi_{x}} \equiv_{n^{*}}\left(\mathrm{EA}^{+}\right)_{n_{0}}^{\alpha_{0}}+\ldots+\left(\mathrm{EA}^{+}\right)_{n^{*}}^{\alpha_{n^{*}}} \equiv_{n}\left(\mathrm{EA}^{+}\right)_{n_{0}}^{\alpha_{0}}+\ldots+\left(\mathrm{EA}^{+}\right)_{n}^{e^{n^{*}-n}\left(\alpha_{n^{*}}\right)}$

b) $\mathrm{Th}_{\psi_{y}} \equiv_{m^{*}}\left(\mathrm{EA}^{+}\right)_{m_{0}}^{\beta_{0}}+\ldots+\left(\mathrm{EA}^{+}\right)_{m^{*}}^{\beta_{m^{*}}} \equiv_{n}\left(\mathrm{EA}^{+}\right)_{m_{0}}^{\beta_{0}}+\ldots+\left(\mathrm{EA}^{+}\right)_{n}^{e^{m^{*}-n}\left(\beta_{m^{*}}\right)}$.

Furthermore, we can make the following observations:

- $e^{n^{*}-n}\left(\alpha_{n^{*}}\right)=x_{n^{*}}=y_{n^{*}}=e^{m^{*}-n}\left(\beta_{m^{*}}\right) ;$ 
- Since for all $m<n$ we have that $x_{m}=y_{m}$, for any $n_{i}<n$ such that $n_{i} \in \mathrm{N}-\bmod \left(\psi_{x}\right)$, provably in $\mathrm{EA}^{+}$:

$$
\left(\mathrm{EA}^{+}\right)_{n_{i}}^{\alpha_{n_{i}}} \subseteq\left(\mathrm{EA}^{+}\right)_{m_{0}}^{\beta_{0}}+\ldots+\left(\mathrm{EA}^{+}\right)_{n}^{e^{m^{*}-n}\left(\beta_{m^{*}}\right)} .
$$

Analogously, for any $m_{i}<n$ such that $m_{i} \in \mathrm{N}-\bmod \left(\psi_{y}\right)$, we have that:

$$
\left(\mathrm{EA}^{+}\right)_{m_{i}}^{\beta_{m_{i}}} \subseteq\left(\mathrm{EA}^{+}\right)_{n_{0}}^{\alpha_{0}}+\ldots+\left(\mathrm{EA}^{+}\right)_{n}^{e^{n^{*}-n}\left(\alpha_{n^{*}}\right)}
$$

Thus, we have that

$$
\begin{aligned}
\mathrm{Th}_{\psi_{x}} & \equiv{ }_{n}\left(\mathrm{EA}^{+}\right)_{n_{0}}^{\alpha_{0}}+\ldots+\left(\mathrm{EA}^{+}\right)_{n}^{e^{n^{*}-n}\left(\alpha_{n^{*}}\right)} \\
& \equiv\left(\mathrm{EA}^{+}\right)_{m_{0}}^{\beta_{0}}+\ldots+\left(\mathrm{EA}^{+}\right)_{n}^{e^{m^{*}-n}\left(\beta_{m^{*}}\right)} \\
& \equiv_{n} \mathrm{Th}_{\psi_{y}} .
\end{aligned}
$$

For the right-to-left implication, assume $\mathrm{Th}_{\psi_{x}} \equiv_{n} \mathrm{Th}_{\psi_{y}}$ and suppose towards a contradiction that there is $m<n$ such that $x_{m} \neq y_{m}$. W.l.o.g assume that $x_{m}>y_{m}$. Hence, we can observe that $\operatorname{Con}_{m}\left(\left(\mathrm{EA}^{+}\right)_{m}^{y_{m}}\right)$ is a $\Pi_{n+1}^{0}$-formula that by Gödel's second incompleteness theorem is not provable in $\mathrm{Th}_{\psi_{y}}$ while remains provable in $\mathrm{Th}_{\psi_{x}}$. This argument can be formalized in $\mathrm{EA}^{+}$which contradicts our assumption.

\subsection{A calculus without normal forms}

This section is dedicated to introduce a presentation of TSC that makes no use of formulas in monomial normal form. To this purpose, we shall make use of INF's, define the notion of $m$ - $\beta$-ordinals and replace Schmerl's axiom by a new principle that establishes the derivability between INF's.

Definition 5.2.1. Let $\left\langle n^{\alpha}\right\rangle A \in I N F, m<n$ and $\beta<\Lambda$. By $o_{m}^{\beta}\left(\left\langle n^{\alpha}\right\rangle A\right)$ we denote the $m$ - $\beta$-ordinal of $\left\langle n^{\alpha}\right\rangle A$, that is recursively defined as follows:

I) $o_{m}^{\beta}\left(\left\langle n^{\alpha}\right\rangle \top\right)=e^{n-m}(\alpha) \cdot(1+\beta)$;

II) $o_{m}^{\beta}\left(\left\langle n^{\alpha}\right\rangle A\right)=e^{n-m}\left(o_{n}^{\alpha}(A)\right) \cdot(1+\beta)$.

For any $m<\omega$ and $\beta<\Lambda$, we set $o_{m}^{\beta}(\top)$ to be zero.

By TSC $^{*}$ we denote the system obtained by substituting in TSC the Schmerl axiom by the following principle:

$$
\text { IW axioms: }\left\langle n^{\alpha}\right\rangle A \equiv\left\langle n_{n}^{\alpha_{n}^{\alpha}(A)}\right\rangle \top \wedge A
$$

for $\left\langle n^{\alpha}\right\rangle A \in$ INF. 


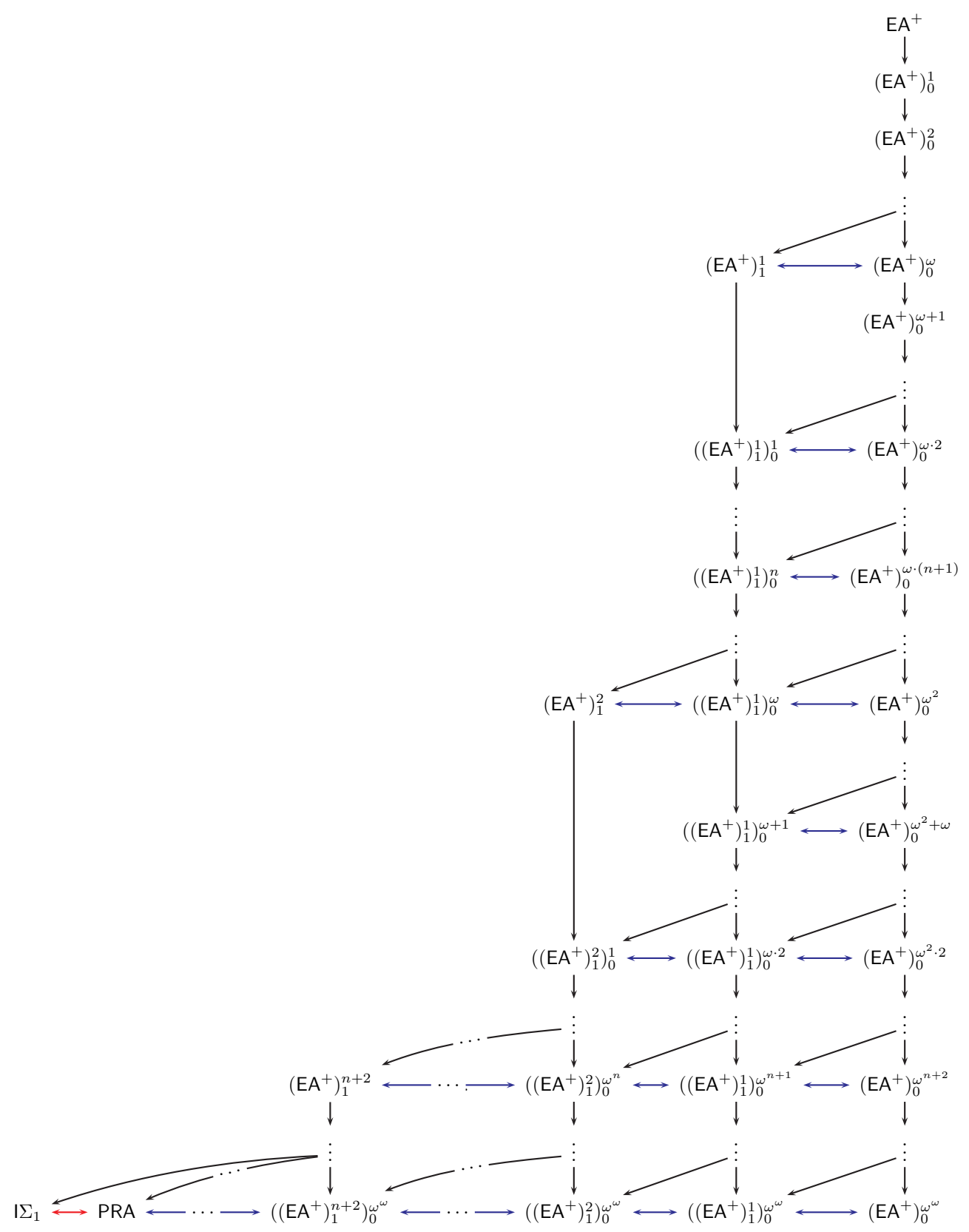

Figure 5.1: A fragment of the road map generated by frame $\mathcal{H}$. The black single-headed arrows $(\rightarrow)$ represent the inclusion between theories $(\subseteq)$. The blue and red double-headed arrows represent the $\Pi_{1}^{0}$ - and $\Pi_{2}^{0}$-conservativity relations, respectively. The equivalences $\left(\mathrm{EA}^{+}\right)_{2}^{1} \equiv \mid \Sigma_{1}$ and $\left(\mathrm{EA}^{+}\right)_{1}^{\omega} \equiv \mathrm{PRA}$ are displayed in the picture. 


\section{MNF's and INF's}

To prove the equivalence of both systems, first we shall see how formulas in monomial normal form and increasing worms are related. Therefore, in the following lemmata we state how every formula in monomial normal form is equivalent to an increasing worm, modulo TSC*, and likewise, that every increasing worm is TSC-equivalent to a formula in monomial normal form.

From now on we will use the following notation: given $\varphi, \psi \in \mathbb{F}_{\mathrm{TSC}}$, we write $\varphi \vdash_{\text {TSC }} \psi\left(\varphi \vdash_{\text {TSC }^{*}} \psi\right)$ to denote that the sequent $\varphi \vdash \psi$ is derivable in TSC (TSC $\left.{ }^{*}\right)$. Analogously, we use $\varphi \equiv_{\text {TSC }} \psi\left(\varphi \equiv_{\text {TSC }^{*}} \psi\right)$ to denote that both $\varphi \vdash \psi$ and $\psi \vdash \varphi$ are derivable in TSC (TSC ${ }^{*}$.

Lemma 5.2.2. For every $\psi:=\left\langle n_{0}^{\alpha_{0}}\right\rangle \top \wedge \ldots \wedge\left\langle n_{k}^{\alpha_{k}}\right\rangle \top \in M N F$ there is an $A \in$ INF such that:

1. $A \equiv_{T_{S S}} \psi$;

2. $A:=\left\langle n_{0}^{\beta_{0}}\right\rangle \ldots\left\langle n_{k}^{\beta_{k}}\right\rangle \top$ where:

a) $\beta_{k}=\alpha_{k}$ and

b) for all $i, 0 \leq i<k$ we have

$$
\beta_{i}=-1+\frac{\alpha_{i}}{e^{n_{i+1}-n_{i}}\left(\alpha_{i+1}\right)}
$$

Proof. By induction on $k$. The base case is trivial and the inductive case follows from the I.H. and the IW axiom.

Recall that by Corollary 3.3.7, for each formula $\varphi \in \mathbb{F}_{\text {TSC }}$ there is a unique $\psi \in$ MNF such that $\varphi \equiv \psi$. Thus, in particular, any $A \in$ INF has its corresponding $\psi \in$ MNF. This combined with Theorem 2.3.2 gives us the following lemma.

Lemma 5.2.3. For any $A:=\left\langle n_{0}^{\beta_{0}}\right\rangle \ldots\left\langle n_{k}^{\beta_{k}}\right\rangle \top \in I N F$ there is a unique $\psi \in M N F$ such that:

1. $\psi \equiv_{T S C} A$;

2. $\psi:=\left\langle n_{0}^{\alpha_{0}}\right\rangle \top \wedge \ldots \wedge\left\langle n_{k}^{\alpha_{k}}\right\rangle \top$ where:

a) $\alpha_{k}=\beta_{k}$ and

b) for all $i, 0 \leq i<k$ we have

$$
\alpha_{i}=o_{n_{i}}^{\beta_{i}}\left(\left\langle n_{i+1}^{\beta_{i+1}}\right\rangle \ldots\left\langle n_{k}^{\beta_{k}}\right\rangle \top\right) .
$$




\section{Equivalence between TSC* and TSC}

We are ready to prove the equivalence of both systems by checking the interderivability of Schmerl and IW axioms.

Proposition 5.2.4. The Schmerl axiom is derivable in TSC$^{*}$ i.e.

$$
\left\langle n^{\alpha}\right\rangle\left(\left\langle n_{0}^{\alpha_{0}}\right\rangle \top \wedge \psi\right) \equiv_{T S C^{*}}\left\langle n^{e^{n_{0}-n}\left(\alpha_{0}\right) \cdot(1+\alpha)}\right\rangle \top \wedge\left\langle n_{0}^{\alpha_{0}}\right\rangle \top \wedge \psi
$$

for $n<n_{0}$ and $\left\langle n_{0}^{\alpha_{0}}\right\rangle \top \wedge \psi \in M N F$.

Proof. By Lemma 5.2.2 we have that:

$$
\left\langle n^{\alpha}\right\rangle\left(\left\langle n_{0}^{\alpha_{0}}\right\rangle \top \wedge \psi\right) \equiv_{\mathbf{T S C}^{*}}\left\langle n^{\alpha}\right\rangle\left\langle n_{0}^{\beta_{0}}\right\rangle A
$$

with $\left\langle n_{0}^{\alpha^{0}}\right\rangle \top \wedge \psi \equiv{ }_{\text {TSC }^{*}}\left\langle n_{0}^{\beta_{0}}\right\rangle A \in$ INF. Thus, by the IW axiom, we get that

$$
\left\langle n^{\alpha}\right\rangle\left(\left\langle n_{0}^{\alpha_{0}}\right\rangle \top \wedge \psi\right) \equiv \text { TSC }^{*}\left\langle n^{o_{n}^{\alpha}}\left(\left\langle n_{0}^{\beta_{0}}\right\rangle A\right)\right\rangle \top \wedge\left\langle n_{0}^{\beta_{0}}\right\rangle A,
$$

and so

$$
\left\langle n^{\alpha}\right\rangle\left(\left\langle n_{0}^{\alpha_{0}}\right\rangle \top \wedge \psi\right) \equiv_{\mathbf{T S C}^{*}}\left\langle n^{o_{n}^{\alpha}}\left(\left\langle n_{0}^{\beta_{0}}\right\rangle A\right)\right\rangle \top \wedge\left\langle n_{0}^{\alpha_{0}}\right\rangle \top \wedge \psi .
$$

Since $o_{n}^{\alpha}\left(\left\langle n_{0}^{\beta_{0}}\right\rangle A\right)=e^{n_{0}-n}\left(o_{n_{0}}^{\beta_{0}}(A)\right) \cdot(1+\alpha)$ and by Lemma 5.2.2, $o_{n_{0}}^{\beta_{0}}(A)=$ $\alpha_{0}$, we can conclude that $o_{n}^{\alpha}\left(\left\langle n_{0}^{\beta_{0}}\right\rangle A\right)=e^{n_{0}-n}\left(\alpha_{0}\right) \cdot(1+\alpha)$ and therefore:

$$
\left\langle n^{\alpha}\right\rangle\left(\left\langle n_{0}^{\alpha_{0}}\right\rangle \top \wedge \psi\right) \equiv_{\mathbf{T S C}^{*}}\left\langle n^{e^{n_{0}-n}\left(\alpha_{0}\right) \cdot(1+\alpha)}\right\rangle \top \wedge\left\langle n_{0}^{\alpha_{0}}\right\rangle \top \wedge \psi .
$$

Proposition 5.2.5. The IW axiom is derivable in TSC i.e.

$$
\left\langle n^{\alpha}\right\rangle A \equiv_{T S C}\left\langle n_{n}^{o_{n}^{\alpha}(A)}\right\rangle \top \wedge A
$$

for $\left\langle n^{\alpha}\right\rangle A \in I N F$.

Proof. By induction on $A$ with base case being trivial. For the inductive step, let $A:=\left\langle n_{0}^{\beta_{0}}\right\rangle A^{\prime}$. By Lemma 5.2.3, we have that

$$
\left\langle n^{\alpha}\right\rangle A \equiv \equiv_{\mathbf{T S C}}\left\langle n^{\alpha}\right\rangle\left(\left\langle n_{0}^{o_{n_{0}}^{\beta_{0}}\left(A^{\prime}\right)}\right\rangle \top \wedge \psi\right)
$$

for $\left\langle n_{0}^{\beta_{0}}\right\rangle A^{\prime} \equiv_{\text {TSC }}\left\langle n_{0}^{o_{n_{0}}^{\beta_{0}}\left(A^{\prime}\right)}\right\rangle \top \wedge \psi \in$ MNF, and so by Schmerl's axiom:

$$
\left\langle n^{\alpha}\right\rangle A \equiv \operatorname{TSC}\left\langle n^{e^{n_{0}-n}}\left(o_{n_{0}}^{\beta_{0}}\left(A^{\prime}\right)\right) \cdot(1+\alpha)\right\rangle \wedge\left\langle n_{0}^{o_{n_{0}}^{\beta_{0}}\left(A^{\prime}\right)}\right\rangle \top \wedge \psi .
$$

Thus,

$$
\left\langle n^{\alpha}\right\rangle A \equiv{ }_{\mathbf{T S C}}\left\langle n^{o_{n}^{\alpha}}\left(\left\langle n_{0}^{\beta_{0}}\right\rangle A^{\prime}\right)\right\rangle \wedge\left\langle n_{0}^{o_{n_{0}}^{\beta_{0}}\left(A^{\prime}\right)}\right\rangle \top \wedge \psi,
$$

that is, $\left\langle n^{\alpha}\right\rangle A \equiv \mathbf{T S C}\left\langle n^{o_{n}^{\alpha}}\left(\left\langle n_{0}^{\beta_{0}}\right\rangle A^{\prime}\right)\right\rangle \wedge A$. 
Corollary 5.2.6. For any $\varphi, \psi \in \mathbb{F}_{T S C}$,

$$
\varphi \vdash_{T S C} \psi \Longleftrightarrow \varphi \vdash_{T S C} \psi
$$

Proof. By induction on the length of the proof. It follows immediately from Propositions 5.2.4 and 5.2.5.

\subsection{Modal Schmerl principles}

In this subsection we give a modal formulation of the Schmerl's formulas introduced in Proposition 3.2.5. We provide a modal proof without using the completeness result.

Proposition 5.3.1. For any $\varphi \in \mathbb{F}_{<n+2}$, the following principles are derivable TSC:

1. $\left\langle(n+1)^{\alpha}\right\rangle \varphi \equiv_{n}\left\langle n^{e(\alpha)}\right\rangle \varphi ;$

2. $\left\langle n^{\alpha}\right\rangle\left\langle(n+1)^{\beta}\right\rangle \varphi \equiv_{n}\left\langle n^{e(\beta) \cdot(1+\alpha)}\right\rangle \varphi$.

Proof. We only check Item 2 since Item 1 follows from Item 2 by taking $\alpha=0$. Let $\psi \in$ MNF such that $\psi \equiv \varphi$. First we prove that the sequent

$$
\left\langle n^{\alpha}\right\rangle\left\langle(n+1)^{\beta}\right\rangle \varphi \vdash\left\langle n^{e(\beta) \cdot(1+\alpha)}\right\rangle \varphi
$$

is derivable. By Theorem 2.3.10 and monotonicity we have that:

$$
\begin{aligned}
&\left\langle n^{\alpha}\right\rangle\left\langle(n+1)^{\beta}\right\rangle \varphi \vdash\left(\bigwedge_{0 \leq i \leq k}\left\langle n_{i}^{\alpha_{i}+e^{n-n_{i}}(e(\beta) \cdot(1+\alpha))}\right\rangle \top\right) \wedge \\
&\left\langle n^{\gamma+e(\beta) \cdot(1+\alpha)}\right\rangle \top \wedge\left\langle(n+1)^{\delta}\right\rangle \top
\end{aligned}
$$

where each $\left\langle n_{i}^{\alpha_{i}}\right\rangle \top$ occurs in $\psi$ with $n_{k}<n$, and $\gamma$ and $\delta$ might be 0 . Thus, by (PSI) we get that $\left\langle n^{\alpha}\right\rangle\left\langle(n+1)^{\beta}\right\rangle \varphi \vdash\left\langle n^{e(\beta) \cdot(1+\alpha)}\right\rangle \varphi$.

For the other direction, consider $\chi \in \mathbb{F}_{n+1}$ such that $\left\langle n^{\alpha}\right\rangle\left\langle(n+1)^{\beta}\right\rangle \varphi \vdash$ $\chi$. Therefore, by (PS1) and (PS2) get the following:

$$
\begin{aligned}
& \left(\bigwedge_{0 \leq i \leq k}\left\langle n_{i}^{\alpha_{i}+e^{n+1-n_{i}}(\delta+\beta)+e^{n-n_{i}}(\alpha)}\right\rangle \top\right) \wedge \\
& \left\langle n^{\gamma+e(\delta+\beta)+e(\delta+\beta) \cdot \alpha}\right\rangle \top \wedge\left\langle(n+1)^{\delta+\beta}\right\rangle \top \vdash \chi
\end{aligned}
$$

where as before, each $\left\langle n_{i}^{\alpha_{i}}\right\rangle \top$ occurs in $\psi$ with $n_{k}<n$, and $\gamma$ and $\delta$ might be 0 . Thus,

$$
\left\langle n_{0}^{\kappa_{0}}\right\rangle \top \wedge \ldots \wedge\left\langle n_{k}^{\kappa_{k}}\right\rangle \top \wedge\left\langle n^{\gamma+e(\delta+\beta) \cdot(1+\alpha)}\right\rangle \top \wedge\left\langle(n+1)^{\delta+\beta}\right\rangle \top \vdash \chi
$$


where $\kappa_{k}:=\alpha_{k}+e^{n-n_{k}}(\gamma+e(\delta+\beta) \cdot(1+\alpha))$ and $\kappa_{i}:=\alpha_{i}+e^{n_{i+1}-n_{i}}\left(\kappa_{i+1}\right)$. We can easily check that there is $\left\{n_{0}, \ldots, n_{j}\right\} \subseteq \mathrm{N}-\bmod \left(\left\langle n_{0}^{\kappa_{0}}\right\rangle \top \wedge \ldots \wedge\right.$ $\left.\left\langle n_{k}^{\kappa_{k}}\right\rangle \top\right)^{1}$ such that:

$$
\left\langle n_{0}^{\kappa_{0}}\right\rangle \top \wedge \ldots \wedge\left\langle n_{j}^{\kappa_{j}}\right\rangle \top \wedge\left\langle n^{\gamma+e(\delta+\beta) \cdot(1+\alpha)}\right\rangle \top \wedge\left\langle(n+1)^{\delta+\beta}\right\rangle \top \vdash \chi
$$

and $\left\langle n_{0}^{\kappa_{0}}\right\rangle \top \wedge \ldots \wedge\left\langle n_{j}^{\kappa_{j}}\right\rangle \top \wedge\left\langle n^{\gamma+e(\delta+\beta) \cdot(1+\alpha)}\right\rangle \top \wedge\left\langle(n+1)^{\delta+\beta}\right\rangle \top \in$ MNF. Let $\chi^{\prime} \in$ MNF such that $\chi^{\prime} \equiv \chi$. Then, with the help of Proposition 3.3.10 we get that:

$$
\left\langle n_{0}^{\kappa_{0}}\right\rangle \top \wedge \ldots \wedge\left\langle n_{j}^{\kappa_{j}}\right\rangle \top \wedge\left\langle n^{\gamma+e(\delta+\beta) \cdot(1+\alpha)}\right\rangle \top \vdash \chi^{\prime} .
$$

Finally, notice that

$$
\left\langle n^{e(\beta) \cdot(1+\alpha)}\right\rangle \psi \vdash\left\langle n_{0}^{\kappa_{0}}\right\rangle \top \wedge \ldots \wedge\left\langle n_{k}^{\kappa_{k}}\right\rangle \top \wedge\left\langle n^{\gamma+e(\delta+\beta) \cdot(1+\alpha)}\right\rangle \top .
$$

This way, $\left\langle n^{e(\beta) \cdot(1+\alpha)}\right\rangle \psi \vdash\left\langle n_{0}^{\kappa_{0}}\right\rangle \top \wedge \ldots \wedge\left\langle n_{j}^{\kappa_{j}}\right\rangle \top \wedge\left\langle n^{\gamma+e(\delta+\beta) \cdot(1+\alpha)}\right\rangle \top$ and so

$$
\left\langle n^{e(\beta) \cdot(1+\alpha)}\right\rangle \psi \vdash \chi^{\prime}
$$

i.e. $\left\langle n^{e(\beta) \cdot(1+\alpha)}\right\rangle \varphi \vdash \chi$.

${ }^{1}$ Observe that this subset may be empty. 


\section{CHAPTER}

\section{The Bracket Calculus}

The content of this chapter is focused on the relation between provability logics and the ordinal notation systems that emerge from them. As raised in the introduction, Beklemishev introduced an ordinal notation system for the Feferman-Schütte ordinal $\Gamma_{0}$ based on the autonomous expansion of provability algebras (see [7]). In this chapter we shall present the logic BC (for Bracket Calculus). The main feature of $\mathbf{B C}$ is that it is based on a signature that extends Beklemishev's brackets notation system to a strictly positive modal language. Thus, unlike other provability logics, BC does not rely on using modalities indexed by some ordinal given a priori but it is built up from a purely modal signature signature. Moreover, since the order between these notations can be established in terms of derivability within the calculus, the inferences in this system can be carried out without using any external property of ordinals. In this sense, we say that BC provides an autonomous provability calculus that to the best of our knowledge, yields the first ordinal notation system presented as a purely modal deductive system.

The presented logic is proven to be equivalent to $\mathbf{R C}_{\Gamma_{0}}$. The main results of this chapter can be found in [21].

\subsection{Beklemishev's bracket notation system for $\Gamma_{0}$}

Before we introduce the full bracket calculus, let us review Beklemishev's notation system from [7]. We start by defining the set of bracket expressions.

Definition 6.1.1. By $\mathbb{W}_{()}$we denote the smallest set such that:

1. $T \in \mathbb{W}_{()}$;

2. if $a, b \in \mathbb{W}_{()}$, then ( $\left.a\right) b \in \mathbb{W}_{()}$. 
By convention we shall write () $a$, for $a \in \mathbb{W}_{()}$to the denote $(T) a \in$ $\mathbb{W}_{()}$.

We can define a translation $*: \mathbb{W}_{()} \rightarrow \mathbb{W}$ in such a way that the elements in $\mathbb{W}_{()}$will denote the ordinals indexing the modalities in $\mathbb{W}$ :

1. $\top^{*}=\top$

2. $((a) b)^{*}=\left\langle o\left(a^{*}\right)\right\rangle b^{*}$.

Therefore, we can also define $o^{*}: \mathbb{W}_{()} \rightarrow \Gamma_{0}$ as $o^{*}(a)=o\left(a^{*}\right)$. Thus the worm $\langle 0\rangle \top$ becomes () and $o^{*}(())=1,\langle 1\rangle \top$ becomes $(())$ with $o^{*}((()))=\omega,\langle\omega\rangle \top$ becomes $((()))$ that corresponds to the ordinal $\varepsilon_{0}$, etc.

Next we make some observations about how the ordinals represented by worms in $\mathbb{W}_{()}$can be bounded in terms of the maximum number of nested brackets occurring in them. With this purpose, we introduce the following two definitions.

Definition 6.1.2. For $a \in \mathbb{W}_{(}$, we define the nesting of $a, N(a)$, as the maximum number of nested brackets. That is:

1. $N(\top)=0$;

2. $N((a) b)=\max (N(a)+1, N(b))$.

Definition 6.1.3. We recursively define the function $h: \mathbb{N} \rightarrow \Gamma_{0}$ as follows:

1. $h(0)=0$;

2. $h(n+1)=e^{h(n)} 1$.

Note that $\lim _{n \rightarrow \infty} h(n)=\Gamma_{0}$.

In the following proposition we can find upper and lower bounds for any ordinal $o^{*}(a)$, with $a \in \mathbb{W}_{()}$, according to the nesting of $a$.

Proposition 6.1.4. For $a \in \mathbb{W}_{()}$, if $N(a)=n$, then $h(n) \leq o^{*}(a)<h(n+1)$.

Proof. By induction on $n$. If $\boldsymbol{n}=\mathbf{0}$ then we must have $a=T$, hence $h(0)=$ $0=o^{*}(a)<1=h(1)$.

For $n=n^{\prime}+1$, we have that $a=\left(a_{0}\right) \ldots\left(a_{m}\right)$ for some $m \in \omega$. Moreover,

1. $\mathrm{N}\left(a_{i}\right) \leq n^{\prime}$ for $i, 0 \leq i \leq m$;

2. there is $a_{J}$ such that $\mathrm{N}\left(a_{J}\right)=n^{\prime}$. 
Thus by the I.H. we get that $a^{*}=\left\langle\alpha_{0}\right\rangle \ldots\left\langle\alpha_{k}\right\rangle \top$ such that:

1. For each $i, \alpha_{i}<h\left(n^{\prime}+1\right)$;

2. there is $\alpha_{J} \geq h\left(n^{\prime}\right)$.

By Lemma 1.4.14,

$$
o\left(\left\langle h\left(n^{\prime}\right)\right\rangle \top\right) \leq o\left(a^{*}\right)<o\left(\left\langle h\left(n^{\prime}+1\right)\right\rangle \top\right) ;
$$

but by Theorem 1.4.13 $o\left(\left\langle h\left(n^{\prime}\right)\right\rangle \top\right)=e^{h\left(n^{\prime}\right)} 1=h(n)$, while $o\left(\left\langle h\left(n^{\prime}+1\right)\right\rangle \top\right)=$ $e^{h(n)} 1=h(n+1)$, as needed.

As a consequence of this last proposition, we get the following corollaries.

Corollary 6.1.5. For $a \in \mathbb{W}_{()}$, if $N(a)=n$, then $a^{*} \in \mathbb{W}_{h(n)}$.

Corollary 6.1.6. For $a, b \in \mathbb{W}_{()}, o^{*}(a) \geq o^{*}(b) \Rightarrow N(a) \geq N(b)$.

Proof. We reason by contrapositive applying Proposition 6.1.4.

\subsection{Signature}

The set of $\mathbf{B C}$-formulas, $\mathbb{F}_{()}$, is defined by extending $\mathbb{W}_{()}$to a strictly positive signature.

Definition 6.2.1. By $\mathbb{F}_{()}$we denote the set of formulas built-up by the following grammar:

$$
\varphi:=\top|p| \varphi \wedge \psi \mid(a) \varphi \text { for } a \in \mathbb{W}_{()} .
$$

Similarly to RC, BC is based on sequents, i.e. expressions of the form $\varphi \vdash \psi$, where $\varphi, \psi \in \mathbb{F}_{()}$. In addition to this, we will also use $a \succeq b$, for $a, b \in \mathbb{W}_{()}$, to denote that either $a \vdash() b$ or $a \vdash b$ are derivable. Analogously, we will use $a \succ b$ to denote that the sequent $a \vdash() b$ is derivable.

Definition 6.2.2. BC is given by the following set of axioms and rules:

Axioms:

1. $\varphi \vdash \varphi, \varphi \vdash \top$;

2. $\varphi \wedge \psi \vdash \varphi, \varphi \wedge \psi \vdash \psi$;

Rules:

1. If $\varphi \vdash \psi$ and $\varphi \vdash \chi$, then $\varphi \vdash \psi \wedge \chi$; 
2. If $\varphi \vdash \psi$ and $\psi \vdash \chi$, then $\varphi \vdash \chi$;

3. If $\varphi \vdash \psi$ and $a \succeq b$, then (a) $\varphi \vdash(b) \psi$ and (a) (b) $\varphi \vdash(b) \psi$;

4. If $a \succ b$, then (a) $\varphi \wedge(b) \psi \vdash(a)(\varphi \wedge(b) \psi)$.

Although BC and RC look quite similar, there is a main difference between them. As we can see, in BC there is no external reference to ordinals, and thus, every derivation is carried out within the calculus. Let us consider the following example.

Let $n \in \mathbb{N}$. The sequent $\langle\omega\rangle \top \vdash\langle n\rangle \top$ is derivable in $\mathbf{R C}_{\boldsymbol{\Gamma}_{\mathbf{0}}}$ by a simple application of Axiom 4 together with the fact that $\omega>n$. However, this last fact is something that we check outside the calculus. If we would like to mimic this derivation within $\mathbf{B C}^{1}$, we could make use of Rule 3. By Axiom 1, we have that $T \vdash_{\mathbf{B C}} T$, so it suffices to give a derivation of " $\omega>n^{\prime \prime}$ within BC.

For $n=0$, we need to check that $(()) \vdash()$. By Axiom 1, we obtain that the sequents ()$\vdash T$ and $T \vdash T$ are derivable in BC. Thus, we can combine this with the following reasoning:

$$
\text { (Rule } 3) \frac{() \vdash T \quad T \vdash \top}{(()) \vdash()}
$$

If $n=1$, we need to check that $(()) \vdash()()$. First, we can extend the previous reasoning as follows:

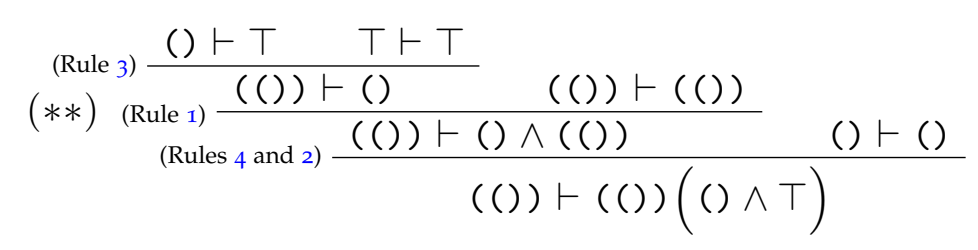

Moreover, we have that

$$
\text { (Rule } 3) \frac{() \wedge T \vdash() \quad() \vdash()}{(())(() \wedge T) \vdash(())()}
$$

Combining these two derivations we obtain that

$$
\text { (Rule 2) } \frac{(()) \vdash(())(() \wedge \top) \quad(())(() \wedge \top) \vdash(())()}{\left(\text { (Rule 3 and 2) } \frac{(()) \vdash(())()}{(()) \vdash()()}(T)\right.}
$$

\footnotetext{
${ }^{1}$ in previous section we shall make precise how to interpret $\mathbf{B C}$-formulas into $\mathbf{R C}_{\boldsymbol{\Gamma}_{\mathbf{0}}}$ formulas and vice versa
} 
In general, by iterating $n$-times this last part of the derivation from where we made an introduction of the conjunction $(* *)$, we obtain that

$$
(()) \vdash \underbrace{() \ldots()()}_{n+1 \text {-times }}
$$

i.e., " $\omega>n$ ". In a similar fashion, applying Rule 3 together with this last derivation we get that

$$
((())) \vdash(\underbrace{() \ldots()()}_{n+1 \text {-times }})
$$

i.e., " $\varepsilon_{0}>e^{n}(1)$ ". These examples illustrate how the required relations between ordinals can be derived within BC without any help of external references.

\subsection{Translation and preservability}

In this section we introduce a way of interpreting $\mathbf{B C}$-formulas as $\mathbf{R C}_{\boldsymbol{\Gamma}_{0}}$ formulas, and prove that under this translation, both systems can derive exactly the same sequents.

Definition 6.3.1. We define a translation $\tau$ between $\mathbb{F}_{()}$and $\mathbb{F}_{\Gamma_{0}}, \tau: \mathbb{F}_{()} \rightarrow \mathbb{F}_{\Gamma_{0}}$, as follows:
1. $\top^{\tau}=\top$;
3. $(\varphi \wedge \psi)^{\tau}=\left(\varphi^{\tau} \wedge \psi^{\tau}\right)$;
2. $p^{\tau}=p$;
4. $((a) \varphi)^{\tau}=\left\langle o^{*}(a)\right\rangle \varphi^{\tau}$.

Note that for $a \in \mathbb{W}_{()}, a^{\tau}=a^{*}$. From this and a routine induction, the following can readily be verified.

Lemma 6.3.2. Given $\varphi \in \mathbb{F}_{()}$and $\alpha \in \mathcal{S}\left(\varphi^{\tau}\right)$, there is a subformula $a \in \mathbb{W}_{()}$of $\varphi$ such that $\alpha=o^{*}(a)$.

The following lemma establishes the preservability of $\mathbf{B C}$ with respect to $\mathbf{R C}_{\Gamma_{0}}$, under $\tau$.

Lemma 6.3.3. For any $\varphi, \psi \in \mathbb{F}_{()}: \varphi \vdash_{B C} \psi \Longrightarrow \varphi^{\tau} \vdash_{\mathbf{R C}_{\Gamma_{0}}} \psi^{\tau}$.

Proof. By induction on the length of the derivation. We can easily check that the set of axioms of $\mathbf{B C}$ is preserved under $\tau$. Likewise, the cases for a derivation ending on Rules 1 or 2 are straightforward. Thus, we only check Rules 3 and 4 .

Regarding Rule 3, we need to prove that if $a \succeq b$ then both sequents $\left\langle o^{*}(a)\right\rangle \varphi^{\tau} \vdash\left\langle o^{*}(b)\right\rangle \psi^{\tau}$ and $\left\langle o^{*}(a)\right\rangle\left\langle o^{*}(b)\right\rangle \varphi^{\tau} \vdash\left\langle o^{*}(b)\right\rangle \psi^{\tau}$ are derivable in $\mathbf{R C}_{\Gamma_{0}}$. We can make the following observations by applying the I.H.: 
1. Since $a \succeq b$, we have that either $a^{\tau} \vdash\langle 0\rangle b^{\tau}$ or $a^{\tau} \vdash b^{\tau}$ are derivable in $\mathbf{R C}_{\Gamma_{0}}$. Therefore, $o\left(a^{\tau}\right) \geq o\left(b^{\tau}\right)$. Since $o^{*}(a)=o\left(a^{*}\right)=o\left(a^{\tau}\right)$ and the same equality holds for $b$, we have that $o^{*}(a) \geq 0^{*}(b)$.

2. We also have that $\varphi^{\tau} \vdash \mathbf{R C}_{\mathbf{\Gamma}_{0}} \psi^{\tau}$ and thus, by Rule 3 of $\mathbf{R C}_{\mathbf{\Gamma}_{0}}$ we obtain that $\left\langle o^{*}(a)\right\rangle \varphi^{\tau} \vdash\left\langle o^{*}(a)\right\rangle \psi^{\tau}$ and $\left\langle o^{*}(a)\right\rangle\left\langle o^{*}(b)\right\rangle \varphi^{\tau} \vdash\left\langle o^{*}(a)\right\rangle\left\langle o^{*}(b)\right\rangle \psi^{\tau}$ are derivable in $\mathbf{R C}_{\Gamma_{0}}$.

On the one hand, by these two facts together with Axiom 4 we obtain that $\left\langle o^{*}(a)\right\rangle \varphi^{\tau} \vdash_{\mathbf{R C}_{\Gamma_{0}}}\left\langle o^{*}(b)\right\rangle \psi^{\tau}$. On the other hand, we can combine Axioms 4 and 3 to get that $\left\langle 0^{*}(a)\right\rangle\left\langle 0^{*}(b)\right\rangle \varphi^{\tau} \vdash_{\mathbf{R C}_{\Gamma_{0}}}\left\langle o^{*}(b)\right\rangle \psi^{\tau}$.

We follow an analogous reasoning in the case of Rule 4. By the I.H. we have that $a^{\tau} \vdash_{\mathbf{R C}_{\mathbf{I}_{0}}}\langle 0\rangle b^{\tau}$. Therefore $o^{*}(a)>o^{*}(b)$ and by Axiom $5,\left\langle o^{*}(a)\right\rangle \varphi \wedge$ $\left\langle o^{*}(b)\right\rangle \psi \vdash \mathbf{R C}_{\Gamma_{0}}\left\langle o^{*}(a)\right\rangle\left(\varphi \wedge\left\langle o^{*}(b)\right\rangle \psi\right)$.

With the following definition we fix a way of translating $\mathbb{F}_{\Gamma_{0}}$-formulas into formulas in $\mathbb{F}_{()}$. However, since different words in $\mathbb{W}_{()}$might denote the same ordinal, we need a normal form theorem for $\mathbb{W}_{()}$.

Definition 6.3.4. We define $N \subset \mathbb{W}_{()}$to be the smallest set of $\mathbb{W}_{()}$-words such that $T \in N$ and for any (a)b $b \mathbb{W}_{()}$, if $a, b \in N$ and $((a) b)^{*} \in B N F$, then (a) $b \in N$.

Every element of $\mathbb{W}_{()}$has a unique normal form, as shown by L. Beklemishev in [7].

Theorem 6.3.5 (Beklemishev). For each $\alpha \in \Gamma_{0}$ we can associate a unique $a_{\alpha} \in N$ such that $o^{*}\left(a_{\alpha}\right)=\alpha$.

Proposition 6.3.6 (Beklemishev). The ordering $\left(N,<_{0}\right)$ is a well-ordering of order type $\Gamma_{0}$.

Now we are ready to translate $\mathbb{F}_{\Gamma_{0}}$-formulas into $\mathbb{F}_{()}$-formulas.

Definition 6.3.7. We define a translation $\iota$ between $\mathbb{F}_{\Gamma_{0}}$ and $\mathbb{F}_{(), \iota:} \mathbb{F}_{\Gamma_{0}} \rightarrow \mathbb{F}_{() \text {, }}$ as follows:
1. $T^{\iota}=\top$;
3. $(\varphi \wedge \psi)^{\iota}=\left(\varphi^{\iota} \wedge \psi^{l}\right)$;
2. $p^{l}=p$;
4. $(\langle\alpha\rangle \varphi)^{\iota}=\left(a_{\alpha}\right) \varphi^{l}$.

The following remark follows immediately from the definitions of $\tau$ and l.

Remark 6.3.8. For any $\varphi \in \mathbb{F}_{\Gamma_{0}},\left(\varphi^{l}\right)^{\tau}=\varphi$.

With the next definition, we extend the nesting $N(a)$ of $a \in W_{()}$to $\mathbb{F}_{()^{-}}$ formulas. 


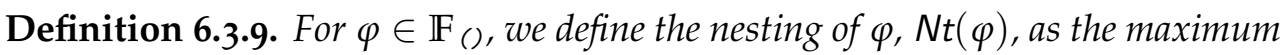
number of nested brackets. That is:

1. $N t(\top)=N t(p)=N(\top)$;

2. $N t(\varphi \wedge \psi)=\max (N t(\varphi), N t(\psi))$;

3. $N t((a) \varphi)=\max (N((a)), N t(\varphi))=\max (N(a)+1, N t(\varphi))$.

The upcoming remark collects a useful observation concerning the nesting $\operatorname{Nt}(\varphi)$ of a formula $\varphi$ and its subformulas. This fact can be verified by an easy induction.

Remark 6.3.10. For any $\varphi \in \mathbb{F}_{()}$with $\varphi \neq p$, there is a subformula $a \in \mathbb{W}_{()}$of $\varphi$ such that $N t(\varphi)=N t(a)$. Moreover, if $N t(\varphi) \geq 1$, there is a subformula $a \in \mathbb{W}_{(}$ of $\varphi$ such that $N t(\varphi)=N t(a)+1$.

For each $\mathbf{R C}_{\Gamma_{0}}$-formula $\varphi$, we can define the signature of $\varphi$ as the set of ordinals occurring in any of its modalities.

Definition 6.3.11. For any $\varphi \in \mathbb{F}_{\Gamma_{0}}$, we define the signature of $\varphi, \mathcal{S}(\varphi)$, as follows:

1. $\mathcal{S}(\top)=\mathcal{S}(p)=\varnothing$;

2. $\mathcal{S}(\varphi \wedge \psi)=\mathcal{S}(\varphi) \cup \mathcal{S}(\psi)$;

3. $\mathcal{S}(\langle\alpha\rangle \varphi)=\{\alpha\} \cup \mathcal{S}(\varphi)$.

With the help of this last definition we can make the following observation:

Lemma 6.3.12. For any $\varphi, \psi \in \mathbb{F}_{\Gamma_{0}}$ :

1. If $\mathcal{S}(\psi) \neq \varnothing$ and $\varphi \vdash \psi$, then $\max \mathcal{S}(\varphi) \geq \max \mathcal{S}(\psi)$;

2. If $\mathcal{S}(\varphi)=\varnothing$ and $\varphi \vdash \psi$, then $\mathcal{S}(\psi)=\varnothing$.

Proof. By an easy induction on the length of the derivation of $\varphi \vdash \psi$.

The following lemma relates the derivability in $\mathbf{R C}_{\boldsymbol{\Gamma}_{0}}$ under $\tau$, and the nesting of formulas in $\mathbb{F}_{()}$.

Lemma 6.3.13. For any $\varphi, \psi \in \mathbb{F}_{()}$:

$$
\varphi^{\tau} \vdash_{\mathbf{R C}_{\mathbf{\Gamma}_{0}}} \psi^{\tau} \Longrightarrow N t(\varphi) \geq N t(\psi) .
$$


Proof. Suppose that $\varphi^{\tau} \vdash{ }_{\mathbf{R C}_{\Gamma_{0}}} \psi^{\tau}$. If $\mathcal{S}\left(\psi^{\tau}\right)=\varnothing$ then it is easy to check that $\operatorname{Nt}(\psi)=0$ and there is nothing to prove, so assume otherwise. Then, by Lemma 6.3.12.1, $\max \mathcal{S}\left(\varphi^{\tau}\right) \geq \max \mathcal{S}\left(\psi^{\tau}\right)$. Using Lemma 6.3.2, let $a \in$ $\mathbb{W}_{()}$be a subformula of $\varphi$ such that $o^{*}(a)=\max \mathcal{S}\left(\varphi^{\tau}\right)$. Moreover, since $\mathcal{S}\left(\psi^{\tau}\right)=\varnothing$, then $\operatorname{Nt}(\psi) \geq 1$. Therefore, with the help of Remark 6.3.10 we can consider $b \in \mathbb{W}_{()}$, a subformula of $\psi$ such that $\mathrm{Nt}(\psi)=\mathrm{N}(b)+$ 1. If we had $\mathrm{N}(a)<\mathrm{N}(b)$ then it would follow from Corollary 6.1.6 that $o^{*}(a)<o^{*}(b)$, contradicting $\max \mathcal{S}\left(\varphi^{\tau}\right) \geq \max \mathcal{S}\left(\varphi^{\tau}\right)$. Thus $\mathrm{N}(a) \geq \mathrm{N}(b)$ and $\mathrm{Nt}(\varphi) \geq \mathrm{N}(a)+1 \geq \mathrm{Nt}(\psi)$, as needed.

With the following theorem we conclude the proof of the preservability between $\mathbf{B C}$ and $\mathbf{R C}_{\mathbf{\Gamma}_{0}}$.

Theorem 6.3.14. For any $\varphi, \psi \in \mathbb{F}_{()}$:

$$
\varphi^{\tau} \vdash_{\mathbf{R C}_{\Gamma_{0}}} \psi^{\tau} \Longleftrightarrow \varphi \vdash_{B C} \psi
$$

Proof. The right-to-left direction is given by Lemma 6.3.3, so we focus on the other. Proceed by induction on $\operatorname{Nt}(\varphi)$. For the base case, assume $\operatorname{Nt}(\varphi)=0$ and $\varphi^{\tau} \vdash{ }_{\mathbf{R C}_{\Gamma_{0}}} \psi^{\tau}$. By a subsidiary induction on the length of the derivation of $\varphi^{\tau} \vdash{ }_{\mathbf{R C}_{\mathbf{I}_{0}}} \psi^{\tau}$, we set to prove $\varphi \vdash_{\mathbf{B C}} \psi$. If the derivation has length one it suffices to check $\mathbf{R C}_{\Gamma_{0}}$-Axioms $I$ and 2, which is immediate. If it has length greater than one it must end in a rule. The case for $\mathbf{R C}_{\Gamma_{0}}$-Rule 1 follows by the I.H.. For $\mathbf{R C}_{\Gamma_{0}}$-Rule 2, we have that there is $\chi \in \mathbb{F}_{\Gamma_{0}}$ such that $\varphi^{\tau} \vdash{ }_{\mathbf{R C}_{\mathbf{I}_{0}}} \chi$ and $\chi \vdash{ }_{\mathbf{R C}_{\mathbf{C}_{0}}} \psi^{\tau}$. By Remark 6.3.8 and Lemma 6.3.13, we get that $\varphi^{\tau} \vdash{ }_{\mathbf{R C}_{\mathbf{\Gamma}_{0}}}\left(\chi^{l}\right)^{\tau}$ and $\left(\chi^{l}\right)^{\tau} \vdash{ }_{\mathbf{R C}_{\mathbf{\Gamma}_{0}}} \psi^{\tau}$ with $\mathrm{Nt}\left(\chi^{l}\right)=0$. Thus, by the subsidiary I.H., $\varphi \vdash_{\mathbf{B C}} \chi^{l}$ and $\chi^{l} \vdash_{\mathbf{B C}} \psi$ and by BC-Rule $2, \varphi \vdash_{\mathbf{B C}} \psi$.

For the inductive step, let $\mathrm{Nt}(\varphi)=n+1$. We proceed by a subsidiary induction on the length of the derivation. If $\varphi^{\tau} \vdash{ }_{\mathrm{RC}_{\Gamma_{0}}} \psi^{\tau}$ is obtained by means of $\mathbf{R C}_{\boldsymbol{\Gamma}_{\mathbf{0}}}$-Axioms $\mathbf{I}$ and 2, then clearly $\varphi \vdash_{\mathbf{B C}} \psi$. If $\varphi^{\tau} \vdash_{\mathbf{R C}_{\mathbf{C}_{0}}} \psi^{\tau}$ is an instance of $\mathbf{R C}_{\mathbf{\Gamma}_{0}}$-Axiom 3, then we have that $\varphi^{\tau}:=\left\langle o^{*}(a)\right\rangle\left\langle o^{*}(b)\right\rangle \chi^{\tau}$ and $\psi^{\tau}:=\left\langle o^{*}(c)\right\rangle \chi^{\tau}$ for some $\chi \in \mathbb{F}_{()}$and $a, b, c \in \mathbb{W}_{()}$such that $o^{*}(a)=$ $o^{*}(b)=o^{*}(c)$. Hence, there are $A, B, C \in \mathbb{W}$ such that $a^{*}=A, b^{*}=B$ and $c^{*}=C$, and so $A \vdash_{\mathbf{R C}_{\Gamma_{0}}} B$ and $B \vdash_{\mathbf{R C}_{\Gamma_{0}}} C$. Since $\mathrm{Nt}(w)<n+1$ for $w \in\{a, b, c\}$, by the main I.H. we have that $a \vdash_{\mathbf{B C}} b$ and $b \vdash_{\mathbf{B C}} c$. Thus, we have the following $\mathbf{B C}$-derivation:

$$
\begin{aligned}
& \text { (Rule 3) } \frac{\chi \vdash \chi \quad b \vdash c}{(b) \chi \vdash(c) \chi} \quad a \vdash b \\
& \quad\left(\text { Rule 3) } \frac{(a)(b) \chi \vdash(b)(c) \chi}{(a)(b) \chi \vdash(c) \chi} \quad \text { (Rule 3) } \frac{\chi \vdash \chi \quad b \vdash c}{(b)(c) \chi \vdash(c) \chi}\right.
\end{aligned}
$$

If $\varphi^{\tau} \vdash \mathbf{R C}_{\mathbf{\Gamma}_{\mathbf{0}}} \psi^{\tau}$ is obtained by using $\mathbf{R C}_{\boldsymbol{\Gamma}_{0}}$-Axiom 4 , then $\varphi^{\tau}:=\left\langle o^{*}(a)\right\rangle \chi^{\tau}$ and $\psi^{\tau}:=\left\langle o^{*}(b)\right\rangle \chi^{\tau}$. for some $\chi \in \mathbb{F}_{()}$and $a, b, \in \mathbb{W}_{()}$with $o^{*}(a)>o^{*}(b)$. Therefore, there are $A, B \in \mathbb{W}_{\Gamma_{0}}$ such that $A \vdash_{\mathbf{R C}_{\Gamma_{0}}}\langle 0\rangle B, a^{*}=A$ and 
$b^{*}=B$. Since $o^{*}(a) \geq o^{*}(() b)$, by Lemma 6.3.12, $\mathrm{Nt}(() b) \leq \mathrm{Nt}(a)$ and since $\varphi^{\tau}:=\left\langle o^{*}(a)\right\rangle \chi^{\tau}$, we have that $\mathrm{Nt}(a)<\mathrm{Nt}(\varphi)$. Thus, by the main I.H. $a \vdash_{\mathbf{B C}}$ () $b$ and by BC-Rule 3, (a) $\chi \vdash_{\mathbf{B C}}(b) \chi$. If $\varphi^{\tau} \vdash_{\mathbf{R C}_{\mathbf{\Gamma}_{\mathbf{0}}}} \psi^{\tau}$ is an instance of $\mathbf{R C}_{\Gamma_{0}}$-Axiom 5, then we have that $\varphi^{\tau}:=\left\langle o^{*}(a)\right\rangle \chi_{0}^{\tau} \wedge\left\langle o^{*}(b)\right\rangle \chi_{1}^{\tau}$ and $\psi^{\tau}:=$ $\left\langle o^{*}(a)\right\rangle\left(\chi_{0}^{\tau} \wedge\left\langle o^{*}(b)\right\rangle \chi_{1}^{\tau}\right)$, for some $\chi_{0}, \chi_{1} \in \mathbb{F}_{()}$and $a, b \in \mathbb{W}_{()}$with $o^{*}(a)>o^{*}(b)$. Therefore, there are $A, B \in \mathbb{W}_{\Gamma_{0}}$ such that $a^{*}=A, b^{*}=B$ and $A \vdash{ }_{\mathbf{R C}_{\Gamma_{0}}}\langle 0\rangle B$. By Lemma 6.3.12 together with the main I.H. we obtain that $a \vdash_{\text {BC }}() b$ and by applying BC-Rule $4,(a) \chi_{0} \wedge(b) \chi_{1} \vdash(a)\left(\chi_{0} \wedge\right.$ (b) $\left.\chi_{1}\right)$. Regarding rules, $\mathbf{R} \mathbf{C}_{\Gamma_{0}}$-Rule 1 is immediate and $\mathbf{R} \mathbf{C}_{\Gamma_{0}}$-Rule 3 follows an analogous reasoning to that of Axiom 4. This way, we only check $\mathbf{R C}_{\Gamma_{0}}$ Rule 2. Assume $\varphi^{\tau} \vdash{ }_{\mathbf{R C}_{\Gamma_{0}}} \psi^{\tau}$ is obtained by an application of $\mathbf{R C}_{\Gamma_{0}}$-Rule 2 . Then, there is $\chi \in \mathbb{F}_{\Gamma_{0}}$ such that $\varphi^{\tau} \vdash_{\mathbf{R C}_{\Gamma_{0}}} \chi$ and $\chi \vdash_{\mathbf{R C}_{\mathbf{I}_{0}}} \psi^{\tau}$. By Remark 6.3.8 together with Lemma 6.3.13 we obtain that $\varphi^{\tau} \vdash{ }_{\mathbf{R C}_{\mathbf{\Gamma}_{\mathbf{0}}}}\left(\chi^{l}\right)^{\tau}$ and $\left(\chi^{l}\right)^{\tau} \vdash{ }_{\mathbf{R C}_{\mathbf{I}_{\mathbf{0}}}}$ $\psi^{\tau}$ with $\mathrm{Nt}(\chi) \leq n+1$. By the subsidiary I.H. $\varphi \vdash_{\mathbf{B C}} \chi^{l}$ and $\chi^{l} \vdash_{\mathbf{B C}} \psi$ and hence, by BC-Rule 2, $\varphi \vdash_{\text {BC }} \psi$.

With this we obtain our main result: an autonomous calculus for representing ordinals below $\Gamma_{0}$.

Theorem 6.3.15. For $a, b \in N$ define $a \triangleleft b$ if and only if $a \vdash_{\mathbf{B C}}() b$. Then, $\triangleleft$ is $a$ strict linear order of order-type $\Gamma_{0}$.

Proof. By Theorem 6.3.14, $a \triangleleft b$ if and only if $a^{\tau} \vdash{ }_{\mathbf{R C}_{\Gamma_{0}}}\langle 0\rangle b^{\tau}$ if and only if $o^{*}(a)<o^{*}(b)$. Moreover if $\xi<o^{*}(a)$ then there is some $B<_{0} a^{\tau}$ such that $\xi=o(B)$, hence $\xi=o^{*}\left(B^{l}\right)$. Thus by Lemma 1.2.4 $o^{*}$ is the order-type function on $\mathrm{N}$. That the range of $o^{*}$ is $\Gamma_{0}$ follows from Proposition 6.1.4 which tells us that $o^{*}(a)<h(\mathrm{~N}(a)+1)<\Gamma_{0}$ for all $a \in \mathbb{W}_{()}$, while if we define recursively $a_{0}=\top$ and $a_{n+1}=\left(a_{n}\right)$, Theorem 1.4.13 and an easy induction readily yield $\Gamma_{0}=\lim _{n \rightarrow \infty} h(n)=\lim _{n \rightarrow \infty} 0^{*}\left(a_{n}\right)$. 



\section{Future Work}

In this last chapter we shall collect some of the future directions in which the work presented in this dissertation can be extended. For the most part, the ideas discussed in this last chapter are work in progress and so, they will not be presented in full detail. However, we deem the open problems that came up during the withing of the thesis to be worth mentoning.

\section{Extensions of TSC}

In Chapters 4 and 5, we examine the relations between MNF's and $\ell$ sequences. As we saw, formulas in monomial normal form and $\ell$-sequences of the form $\left\langle x_{0}, \ldots, x_{k}, 0\right\rangle$ can be related. Thus, having finite support is essential. However, when working in a signature with $\Lambda>\varepsilon_{0}$, we shall come across sequences that do not converge to zero e.g. the sequence $\left\langle\varepsilon_{0}, \varepsilon_{0}, \ldots, \varepsilon_{0}, \ldots\right\rangle$.

Non-finitely supported $\ell$-sequences cannot be defined by means of a modal formula. However, we can provide a natural arithmetical interpretation of these sequences.

Given a $\ell$-sequence of the form $\left\langle\varepsilon_{\alpha}, \varepsilon_{\alpha}, \ldots, \varepsilon_{\alpha}, \ldots\right\rangle$, it can be associated to the Turing progression

$$
\bigcup_{n<\omega}\left(\mathrm{EA}^{+}\right)_{n}^{\varepsilon_{\alpha}} .
$$

Thus, the natural candidate to represent this theory in our modal setting would be

$$
\bigwedge_{n<\omega}\left\langle n^{\varepsilon_{\alpha}}\right\rangle \top,
$$

which requires to allow infinite conjunctions.

Another solution to this situation involves extending our current modal signature and making use of the conservativity results about transfinite induction and Turing progressions established by Schmerl in [34].

Let $\operatorname{TI}(\alpha)$ be the transfinite induction scheme up to ordinal $\alpha$ and $\operatorname{TI}_{n}(\alpha)$ the restriction of $\mathrm{TI}(\alpha)$ to $\Pi_{n+1}^{0}$-formulas. $\mathrm{By}\left[\mathrm{EA}^{+}\right]^{\varepsilon_{\alpha}}$ we denote the theory 
$\mathrm{EA}^{+}+\operatorname{TI}\left(\varepsilon_{\alpha}\right)$ and, analogously, by $\left[\mathrm{EA}^{+}\right]_{n}^{\varepsilon_{\alpha}}$ we denote the theory $\mathrm{EA}^{+}+$ $\mathrm{TI}_{n}\left(\varepsilon_{\alpha}\right)$.

The following result captures how transfinite induction can be approximated by means of Turing progressions.

Proposition. For any $n<\omega$, provably in $\mathrm{EA}^{+}$:

1. $\left(\mathrm{EA}^{+}\right)_{n}^{\varepsilon_{\alpha}} \subseteq\left[\mathrm{EA}^{+}\right]^{\varepsilon_{\alpha}}$

2. $\left[\mathrm{EA}^{+}\right]_{n}^{\varepsilon_{\alpha}} \subseteq\left(\mathrm{EA}^{+}\right)_{n+2}^{\varepsilon_{\alpha}}$;

3. $\left[\mathrm{EA}^{+}\right]^{\varepsilon_{\alpha}} \equiv_{n}\left(\mathrm{EA}^{+}\right)_{n}^{\varepsilon_{\alpha}}$.

Therefore, taking back our previous example, we have that

$$
\bigcup_{n<\omega}\left(\mathrm{EA}^{+}\right)_{n}^{\varepsilon_{\alpha}} \equiv\left[\mathrm{EA}^{+}\right]^{\varepsilon_{\alpha}}
$$

A way of making TSC capable of capturing theories like these ones, but at same time, not requiring infinite conjunctions, would be by extending $\mathbb{F}_{\text {TSC }}$ with a new modality $\left[\varepsilon_{\alpha}\right]$ :

Definition. By $\mathbb{F}_{T S C}^{+}$we denote the smallest set such that:

I) $T \in \mathbb{F}_{T S C}^{+}$;

II) If $\varphi, \psi \in \mathbb{F}_{\text {TSC }}^{+}$then $(\varphi \wedge \psi) \in \mathbb{F}_{\text {TSC }}^{+}$;

III) if $\varphi \in \mathbb{F}_{T S C}^{+}, n<\omega$ and $\alpha<\Lambda$ then $\left\langle n^{\alpha}\right\rangle \varphi \in \mathbb{F}_{T S C^{\prime}}^{+}$;

IV) if $\varphi \in \mathbb{F}_{T S C}^{+}$and $\varepsilon_{\alpha}<\Lambda$ then $\left[\varepsilon_{\alpha}\right] \varphi \in \mathbb{F}_{T S C^{\prime}}^{+}$;

The goal of this new modality is to capture the transfinite induction schema up to some $\varepsilon$-number. Thus, formulas of the form $\left[\varepsilon_{\alpha}\right] \varphi$ are intended to be arithmetically interpreted as $\mathrm{Th}_{\varphi}+\mathrm{TI}\left(\varepsilon_{\alpha}\right)$.

The additional axioms and rules that should be added to TSC to obtain a complete calculus capable of generating all the principles that govern the behavior of such theories, remains as an open problem. However, there are some principles that should be derivable in this new system.

First of all, in this setting we are allowed to build progressions over base theories which might have transfinite induction available. To this end, the following conservativity result should be expressible within this new system:

Lemma. For any $\varepsilon_{\alpha}, \beta \prec \Lambda$ and $n<\omega$, provably in $\mathrm{EA}^{+}$:

$$
\left(\left[\mathrm{EA}^{+}\right]^{\varepsilon_{\alpha}}\right)_{n}^{\beta} \equiv_{n}\left(\mathrm{EA}^{+}\right)_{n}^{\varepsilon_{\alpha} \cdot(1+\beta)} .
$$

96 
Proof. Reasoning in $\mathrm{EA}^{+}$, since $\left[\mathrm{EA}^{+}\right]^{\varepsilon_{\alpha}} \equiv_{n+1}\left(\mathrm{EA}^{+}\right)_{n+1}^{\varepsilon_{\alpha}}$, by a combination of Corollary 3.2.12, Proposition 3.2.5, Item 3.2.5 and the fact that $\omega^{\varepsilon_{\alpha}}=\varepsilon_{\alpha}$, we obtain that $\left(\left[\mathrm{EA}^{+}\right]^{\varepsilon_{\alpha}}\right)_{n}^{\beta} \equiv_{n}\left(\mathrm{EA}^{+}\right)_{n}^{\varepsilon_{\alpha} \cdot(1+\beta)}$.

Thus, there are some modal principles that should be derivable:

- $\left[\varepsilon_{\alpha}\right] \top \vdash\left\langle n^{\varepsilon_{\alpha}}\right\rangle \top$ for any $n<\omega$;

- $\left\langle n^{\beta}\right\rangle\left[\varepsilon_{\alpha}\right] \top \vdash\left\langle n^{\varepsilon_{\alpha} \cdot(1+\beta)}\right\rangle \top$;

- $\left[\varepsilon_{\alpha}\right]\left[\varepsilon_{\beta}\right] \top \equiv\left[\varepsilon_{\max (\alpha, \beta)}\right] \top$.

On the other hand, since Schmerl Axioms are the keystone of TSC, we are confident that a modal version of the following formulation of the finestructure formula for theories $\left[\mathrm{EA}^{+}\right]^{\varepsilon_{\alpha}}$ might play the role of the Schmerl Axioms for this extended version of TSC.

Proposition. For any $\varepsilon_{\alpha}, \beta, \gamma \prec \Lambda$, and $n<\omega$, provably in $\mathrm{EA}^{+}$:

1. $\left(\left[\mathrm{EA}^{+}\right]^{\varepsilon_{\alpha}}\right)_{n+1}^{\beta} \equiv_{n}\left(\left[\mathrm{EA}^{+}\right]^{\varepsilon_{\alpha}}\right)_{n}^{e^{1}\left(\varepsilon_{\alpha} \cdot \beta\right)}$;

2. $\left(\left(\left[\mathrm{EA}^{+}\right]^{\varepsilon_{\alpha}}\right)_{n+1}^{\beta}\right)_{n}^{\gamma} \equiv_{n}\left(\left[\mathrm{EA}^{+}\right]^{\varepsilon_{\alpha}}\right)_{n}^{e^{1}\left(\varepsilon_{\alpha} \cdot \beta\right) \cdot(1+\gamma)}$.

Proof. Item 1 follows from Item 2 for $\gamma=0$. Thus, we only prove Item 2. Reasoning in $\mathrm{EA}^{+}$we have that

$$
\left(\left[\mathrm{EA}^{+}\right]^{\varepsilon_{\alpha}}\right)_{n}^{e^{1}\left(\varepsilon_{\alpha} \cdot \beta\right) \cdot(1+\gamma)} \equiv_{n}\left(\mathrm{EA}^{+}\right)_{n}^{\varepsilon_{\alpha} \cdot\left(e^{1}\left(\varepsilon_{\alpha} \cdot \beta\right) \cdot(1+\gamma)\right)} .
$$

We can observe that $e^{1}\left(\varepsilon_{\alpha} \cdot \beta\right) \cdot(1+\gamma)=\varepsilon_{\alpha}{ }^{\beta} \cdot(1+\gamma)$. Therefore,

$$
\varepsilon_{\alpha} \cdot\left(e^{1}\left(\varepsilon_{\alpha} \cdot \beta\right) \cdot(1+\gamma)\right)=\varepsilon_{\alpha} \cdot\left(\varepsilon_{\alpha}^{\beta} \cdot(1+\gamma)\right)=e^{1}\left(\varepsilon_{\alpha} \cdot(1+\beta)\right) \cdot(1+\gamma) .
$$

On the other hand, we have that:

$$
\left(\left[\mathrm{EA}^{+}\right]^{\varepsilon_{\alpha}}\right)_{n+1}^{\beta} \equiv_{n+1}\left(\mathrm{EA}^{+}\right)_{n+1}^{\varepsilon_{\alpha} \cdot(1+\beta)}
$$

and

$$
\left(\left(\left[\mathrm{EA}^{+}\right]^{\varepsilon_{\alpha}}\right)_{n+1}^{\beta}\right)_{n}^{\gamma} \equiv_{n}\left(\left(\mathrm{EA}^{+}\right)_{n+1}^{\varepsilon_{\alpha} \cdot(1+\beta)}\right)_{n}^{\gamma} \equiv_{n}\left(\mathrm{EA}^{+}\right)_{n}^{e^{1}}\left(\varepsilon_{\alpha^{*}} \cdot(1+\beta)\right) \cdot(1+\gamma) .
$$

Combining all these facts we get that

$$
\begin{aligned}
\left(\left(\left[\mathrm{EA}^{+}\right]^{\varepsilon_{\alpha}}\right)_{n+1}^{\beta}\right)_{n}^{\gamma} & \equiv_{n}\left(\mathrm{EA}^{+}\right)_{n}^{e^{1}\left(\varepsilon_{\alpha} \cdot(1+\beta)\right) \cdot(1+\gamma)} \\
& \equiv_{n}\left(\mathrm{EA}^{+}\right)_{n}^{\varepsilon_{\alpha} \cdot\left(e^{1}\left(\varepsilon_{\alpha} \cdot \beta\right) \cdot(1+\gamma)\right)} \\
& \equiv_{n}\left(\left[\mathrm{EA}^{+}\right]^{\varepsilon_{\alpha}}\right)_{n}^{e^{1}\left(\varepsilon_{\alpha} \cdot \beta\right) \cdot(1+\gamma)}
\end{aligned}
$$


From the relational semantics point of view, this new modality seems to have a natural interpretation in our frame $\mathcal{J}$ :

$$
\mathcal{J}, x \Vdash\left[\varepsilon_{\alpha}\right] \varphi \text { iff there is } y \in I \text { such that } \mathcal{J}, y \Vdash \varphi \text { and } \forall n<\omega x R_{n}^{\varepsilon_{\alpha}} y \text {. }
$$

This interpretation allow us to relate non-finitely supported $\ell$-sequences to modal formulas and ultimately, to arithmetical theories in the obvious way:

$$
\begin{array}{|c|c|c|}
\hline\left\langle\varepsilon_{0}, \varepsilon_{0}, \ldots, \varepsilon_{0}, \ldots\right\rangle & {\left[\varepsilon_{0}\right] \top} & {\left[\mathrm{EA}^{+}\right]^{\varepsilon_{0}}} \\
\left\langle\varepsilon_{0} \cdot 2, \varepsilon_{0}, \ldots, \varepsilon_{0}, \ldots\right\rangle & \left\langle 0^{1}\right\rangle\left[\varepsilon_{0}\right] \top & \left(\left[\mathrm{EA}^{+}\right]^{\varepsilon_{0}}\right)_{0}^{1} \\
\left\langle\varepsilon_{0}^{2}, \varepsilon_{0} \cdot 2, \ldots, \varepsilon_{0}, \ldots\right\rangle & \left\langle 1^{1}\right\rangle\left[\varepsilon_{0}\right] \top & \left(\left[\mathrm{EA}^{+}\right]^{\varepsilon_{0}}\right)_{1}^{1} \\
\left\langle\varepsilon_{0}^{2} \cdot 2, \varepsilon_{0} \cdot 2, \ldots, \varepsilon_{0}, \ldots\right\rangle & \left\langle 0^{1}\right\rangle\left\langle 1^{1}\right\rangle\left[\varepsilon_{0}\right] \top & \left(\left(\left[\mathrm{EA}^{+}\right]^{\varepsilon_{0}}\right)_{1}^{1}\right)_{0}^{1} \\
\hline
\end{array}
$$

In a different vein, in Chapter 3, Section 3.3 we introduce Corollary 3.3.1 to be the only tool to obtain non-derivability in TSC. We have chosen to do so since Corollary 3.3.1 can also be obtained via relational semantics. Consequently, we shall be able to generalize the entire purely modal results of this dissertation to a setting where we work with ordinal modalities where the base elements are also transfinite ordinals.

\section{BC}

In Chapter 6 we gave a purely syntactical analysis of BC which leaves room for a semantical treatment. The equivalence between our system and $\mathbf{R} \mathbf{C}_{\Gamma_{0}}$ allows us to make use of the relational semantics for $\mathbf{R C}_{\boldsymbol{\Gamma}_{0}}$ presented in $[17,9]$, but we leave the question of whether it is possible to define natural semantics that work only with $\mathbf{B C}$ expressions and do not directly reference ordinals. Moreover, [20] suggests variants of the brackets notation for representing the Bachmann-Howard ordinal and beyond. Sound and complete calculi for these systems remain to be found. 


\section{Bibliography}

[1] L. D. Beklemishev. Provability logics for natural Turing progressions of arithmetical theories. Studia Logica, 50(1), 1991.

[2] L. D. Beklemishev. Iterated local reflection versus iterated consistency. Annals of Pure and Applied Logic, 75(1-2), 1995.

[3] L. D. Beklemishev. Another pathological well-ordering. Bulletin of Symbolic Logic, 7(4):534-534, 2001.

[4] L. D. Beklemishev. Proof-theoretic analysis by iterated reflection. Archive for Mathematical Logic, 42(6), 2003.

[5] L. D. Beklemishev. Provability algebras and proof-theoretic ordinals, I. Annals of Pure and Applied Logic, 128(1-3), 2004.

[6] L. D. Beklemishev. Reflection schemes and provability algebras in formal arithmetic. Russian Mathematical Surveys, 6o(2), 2005.

[7] L. D. Beklemishev. Veblen hierarchy in the context of provability algebras. In P. Hájek, L. Valdés-Villanueva, and D. Westerståhl, editors, Logic, Methodology and Philosophy of Science, Proceedings of the Twelfth International Congress, pages 65-78. Kings College Publications, 2005.

[8] L. D. Beklemishev. Calibrating provability logic: from modal logic to reflection calculus. In Advances in modal logic, volume 9, pages 89-94. College Publications, 2012.

[9] L. D. Beklemishev. Positive provability logic for uniform reflection principles. Annals of Pure and Appied Logic, 165(1), 2014.

[10] L. D. Beklemishev. Reflection calculus and conservativity spectra. Russian Mathematical Surveys, 73(4), 2018.

[11] L. D. Beklemishev. A universal algebra for the variable-free fragment of $\mathbf{R C}^{\nabla}$. In Logical Foundations of Computer Science, International Symposium, LFCS 2018, volume 10703 of Lecture Notes in Computer Science, pages 91106, Berlin, Heidelberg, 2018. Springer. 
[12] L. D. Beklemishev, D. Fernández-Duque, and J. J. Joosten. On provability logics with linearly ordered modalities. Studia Logica, 102(3), 2014.

[13] L. D. Beklemishev and D. Gabelaia. Topological completeness of the provability logic GLP. Annals of Pure and Applied Logic, 164(12), 2013.

[14] L. D. Beklemishev, J. J. Joosten, and M. Vervoort. A finitary treatment of the closed fragment of Japaridze's provability logic. Journal of Logic and Computation, 15(4), 2005.

[15] L.D. Beklemishev. On the reflection calculus with partial conservativity operators. In WoLLIC 2017, volume 10388 of Lecture Notes in Computer Science, pages $48-67,2017$.

[16] G. S. Boolos. The Logic of Provability. Cambridge University Press, Cambridge, 1993.

[17] E. V. Dashkov. On the positive fragment of the polymodal provability logic GLP. Mathematical Notes, 91(3), 2012.

[18] A. de Almeida Borges and J. J. Joosten. The worm calculus. In Advances in Modal Logic, volume 12, pages 13-27. College Publications, 2018.

[19] D. Fernández-Duque. The polytopologies of transfinite provability logic. Archive for Mathematical Logic, 53(3-4):385-431, 2014.

[20] D. Fernández-Duque. Worms and spiders: Reflection calculi and ordinal notation systems. Journal of Applied Logics - IfCoLoG Journal of Logics and their Applications, 4(10), 2017.

[21] D. Fernández-Duque and E. Hermo-Reyes. A self-contained provability calculus for $\Gamma_{0}$. In Logic, Language, Information, and Computation WoLLIC 2019, volume 11541 of Lecture Notes in Computer Science, pages 195-207. Springer, 2019.

[22] D. Fernández-Duque and J. J. Joosten. Hyperations, veblen progressions and transfinite iteration of ordinal functions. Annals of Pure and Applied Logic, 164(7-8), 2013.

[23] D. Fernández-Duque and J. J. Joosten. Models of transfinite provability logics. Journal of Symbolic Logic, 78(2), 2013.

[24] D. Fernández-Duque and J. J. Joosten. Well-orders in the transfinite Japaridze algebra. Logic Journal of the IGPL, 22(6), 2014.

[25] D. Fernández-Duque and J. J. Joosten. The omega-rule interpretation of transfinite provability logic. Annals of Pure and Applied Logic, 169(4), 2017. 
[26] K. Gödel. Über formal unentscheidbare sätze der principia mathematica und verwandter systeme I. Monatshefte für Mathematik und Physik, 38,1931 .

[27] E. Hermo-Reyes and J. J. Joosten. The logic of Turing progressions. Accepted for publication in Notre Dame Journal of Formal Logic.

[28] E. Hermo-Reyes and J. J. Joosten. Relational semantics for the Turing Schmerl calculus. In Advances in Modal Logic, volume 12, pages 327-346. College Publications, 2018.

[29] T. F. Icard III. A topological study of the closed fragment of GLP. Journal of Logic and Computation, 21(4), 2011.

[30] K. N. Ignatiev. On strong provability predicates and the associated modal logics. The Journal of Symbolic Logic, 58, 1993.

[31] G. Japaridze. The polymodal provability logic. In Intensional logics and logical structure of theories: material from the Fourth Soviet-Finnish Symposium on Logic. Metsniereba, Telavi, 1988. In Russian.

[32] Joost J. Joosten. Turing-Taylor expansions for arithmetic theories. Studia Logica, 104(6), 2016.

[33] G. Kreisel. Wie die beweistheorie zu ihren ordinalzahlen kam und kommt. Jahresbericht der Deutschen Mathematiker-Vereinigung, 78, 1976/77.

[34] U. R. Schmerl. A fine structure generated by reflection formulas over primitive recursive arithmetic. In Logic Colloquium ' 78 (Mons, 1978), volume 97 of Stud. Logic Foundations Math., pages 335-350. North-Holland, Amsterdam, 1979 .

[35] R. M. Solovay. Provability interpretations of modal logic. Israel Journal of Mathematics, 25(3-4), 1976.

[36] A. Turing. Systems of logics based on ordinals. Proceedings of the London Mathematical Society, 45(2), 1939. 